ALBERTO BELOTTI COLOMBO

Applications of Structural Health Monitoring and Field Testing Techniques to Probabilistic Based Life-Cycle Evaluation of Reinforced Concrete Bridges 
ALBERTO BELOTTI COLOMBO

\section{Applications of Structural Health Monitoring and Field Testing Techniques to Probabilistic Based Life-Cycle Evaluation of Reinforced Concrete Bridges}

Thesis presented to the Polytechnic School of the University of São Paulo in candidacy for the Degree of Doctor of Science 


\section{Applications of Structural Health Monitoring and Field Testing Techniques to Probabilistic Based Life-Cycle Evaluation of Reinforced Concrete Bridges}

Thesis presented to the Polytechnic School of the University of São Paulo in candidacy for the Degree of Doctor of Science

Area of Concentration: Structural Engineering

Advisor: Prof. Dr. Túlio Nogueira Bittencourt 
Este exemplar foi revisado e corrigido em relação à versão original, sob responsabilidade única do autor e com a anuência de seu orientador.

São Paulo, de de

Assinatura do autor:

Assinatura do orientador:

Colombo, Alberto B.

Applications of Structural Health Monitoring and Field Testing Techniques to Probabilistic Based Life-Cycle Evaluation of Reinforced Concrete Bridges / A. B. Colombo -- versão corr. -- São Paulo, 2016. $127 \mathrm{p}$.

Tese (Doutorado) - Escola Politécnica da Universidade de São Paulo. Departamento de Engenharia de Estruturas e Geotécnica.

1.Structural Testing 2.Structural Health Monitoring (SHM) 3.Reinforced Concrete 4.Bridges 5.Life-Cycle I.Universidade de São Paulo. Escola Politécnica. Departamento de Engenharia de Estruturas e Geotécnica II.t. 
To my family... 


\section{Acknowledgments}

I would like to thank my research advisor, Prof. Túlio Nogueira Bittencourt, for the opportunity and guidance that he provided during these years. Without his support this work would not have been possible.

I would also like to express my immense gratitude to Prof. Dan M. Frangopol, for hosting me at Lehigh University. The experiences gathered during that period are priceless and the time spent there was fundamental to the development of this work. I'm also very grateful for his counsel and company during that period.

I'd like to acknowledge CAPES for the financial support during this doctorate. Also, without the support of VALE and Arteris in their research projects, this work would not have been possible.

The never ending support of my parents was also essential during this stage and without it nothing could have been accomplished.

Special thanks goes to my brother for his free consultations and friendship during these not so easy years.

I'd also like to thank my colleagues and friends from the University of São Paulo who endured the hard days of field work and helped in keeping me sane with a couple of beers after each job. A special mention must be made to Alfredo for his endless patience and wise words.

To the friends, colleagues, and many housemates from Bethlehem. Who welcomed me into their lives and made the long periods away from home pass by so fast.

Last but, certainly not least, I'd like to express my deep gratitude to my fiancé Kaciane. Her support was fundamental during this stage of my life. 


\section{Abstract}

This work presents methodologies for the integration of field testing and Structural Health Monitoring SHM in the assessment of reinforced concrete bridges. The methodologies are demonstrated through the use of data collected during the testing of reinforced concrete railway bridges and long-term monitoring of a highway bridge.

A probabilistic life-cycle prediction model based on sectional analysis is proposed for reinforced concrete structures. The updating of the model parameters is done using a Bayesian updating approach in which the problem is defined as a reliability one. An algorithm that uses subset simulation is used to sample points from the updated parameter distributions. Testing data from a reinforced concrete railway bridge is used to demonstrate the methodology and its results.

The description of a SHM system that was installed in the Jaguari River Bridge is also presented. During the stages of preparation for the installation of this system the bridge was inspected, had NDT performed, and field testing was conducted using a test truck. The results of these tests are also presented.

Analysis of the collected data from the live-load response of the Jaguari River Bridge is used to demonstrate methodologies for obtaining live-load response distributions from monitoring data. The use of this live-load response data is also used for the life-cycle analysis of one of the bridge's cross-sections.

Keywords: structural testing; structural health monitoring; reinforced concrete; bridges; life-cycle. 


\section{Resumo}

Este trabalho apresenta metodologias de integração de ensaios estruturais e Structural Health Monitoring (SHM) para a avaliação de pontes de concreto armado. O SHM diz respeito a um conjunto de praticas com o objetivo de acompanhar o comportamento estrutural através de sensores com o objetivo de acompanhar o comportamento da estrutura e determinar ações de manutenção de maneira proativa. As metodologias são apresentadas através do uso de dados coletados durante ensaios de pontes ferroviárias em concreto armado e do monitoramento continuo de uma ponte rodoviária.

Um modelo para o ciclo de vida de estruturas de concreto armado baseado no método das lamelas é proposto. Os parâmetros deste modelo, que são considerados de maneira probabilística, são atualizados através de um método Bayesiano. Dados de ensaios de uma ponte ferroviária são utilizados nesta analise.

A descrição de um sistema de monitoramento contínuo instalado na Ponte do Rio Jaguari também é feita. Durante as etapas de desenvolvimento do sistema a ponte foi inspecionada, ensaios não destrutivos foram feitos e ensaios com um veículo teste foram conduzidos. Os resultados e analises destes também são apresentados.

Os dados coletados por este sistema foram utilizados para demonstrar metodologias de caracterização dos modelos de resposta devido a cargas moveis. A utilização destes modelos na avaliação de confiabilidade ao longo do tempo de uma das seções da ponte também é apresentada.

Palavras-chave: ensaio estrutural; monitoramento estrutural; concreto armado; pontes; ciclo de vida estrutural. 


\section{List of Figures}

2.1. Illustration of the layer-by-layer discretization of RC cross section including the strain and, corresponding stress field across the section depth. . . . . . 20

2.2. Stress-strain curves for steel (left) and for concrete (right). . . . . . . . . . 22

2.3. Bridge geometry and sensor positioning. . . . . . . . . . . . . 31

2.4. Use of measured wheel loads to determine train loading on the bridge. . . . 32

2.5. Bayesian updating results based on the measured displacement data. . . . 35

2.6. Predicted ultimate bending moment of the cross section considering updating with different information. . . . . . . . . . . . . . . 37

2.7. Curves showing: the mean values of the ultimate bending moment $\left(M_{u}\right)$ and the relative damage $\left(M_{u} / M_{u(\text { initial })}\right)$ at different ages. . . . . . . . . 40

3.1. Location of the Jaguari River Bridge. . . . . . . . . . . . . . . . . 44

3.2. Plan view of the Jaguari River Bridge. . . . . . . . . . . . . . . . . 45

3.3. Elevation view of the Jaguari River Bridge. . . . . . . . . . . . . . . 45

3.4. Typical cross-sections of the Jaguari River Bridge. . . . . . . . . . . . . . 46

3.5. Class 45 live loading pattern for the Brazilian standard NBR 7188. . . . . 47

3.6. Shrinkage cracks detected in the longitudinal girders. . . . . . . . . . . . 48

3.7. Flexural cracks found in the longitudinal girders. . . . . . . . . . . . . . 48

3.8. View beneath the deck slab between columns P5 and P6. . . . . . . . . 49

3.9. Crack with infiltration close to column P4 (São Paulo side). . . . . . . . . 49

3.10. Crack with infiltration close to column P4 (Belo Horizonte side): (a) overview, (b) zoomed in. . . . . . . . . . . . . . . . . . . . 50 50

3.11. Visual inspection of the laminated elastomeric bearing pads showing cracking (a) and distortion (b). . . . . . . . . . . . . . 5 50

3.12. Longitudinal view of the short-term instrumentation plan including the displacement sensor and accelerometers. . . . . . . . . . . . . 51

3.13. Displacement sensors installed at the midspans (a), supports (b), cracks in

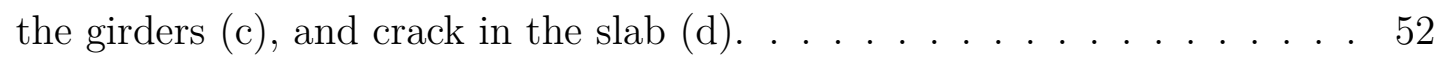

3.14. Installation technique for measuring displacements at the midspan. . . . . 53

3.15. Accelerometer installed at the side of the longitudinal girder. . . . . . . . . 53

3.16. Positioning of the strain gauges, in the analyzed cross-sections, for the short-term monitoring. . . . . . . . . . . . . . . 54 
3.17. Details showing: the uncovered reinforcement with the treated surface for strain gauge application (a); asphalt removal at the top surface for gauge installation (b). . . . . . . . . . . . . . . 55

3.18. Installed strain gauge in the bottom reinforcement (a), and installation of strain gauge at the top of the girder $(\mathrm{b}) \ldots \ldots . . \ldots$. . . . . . . . 55

3.19. Images showing the data acquisition setup. . . . . . . . . . . . . . 55

3.20. Test vehicle that was used (a); and its axle load configuration (b). . . . . 56

3.21. Picture showing the truck position for the series of static tests. . . . . . . . 57

3.22. Aligned strain time-histories, of selected sensors in the girder, for the three static tests. . . . . . . . . . . . . . . . . . 57

3.23. Aligned strain time-histories, of selected sensors in the transverse direction of the deck, for the three static tests. . . . . . . . . . . . . . . . . . 58

3.24. Aligned strain time-histories, of selected sensors in the girders, for the dynamic tests. . . . . . . . . . . . . . . . . . . . . 59

3.25. Aligned strain time-histories, of sensor in the transverse direction of the deck, for the dynamic tests. . . . . . . . . . . . . . . . . . . 59

3.26. Example of collected data in the short-term monitoring. . . . . . . . . . . . 60

3.27. Histograms of maximum strains obtained in the trigger channels for the collected short-term data. . . . . . . . . . . . . . . . . . . . . 62

3.28. Longitudinal view of the long-term monitoring system for the Jaguari River Bridge. . . . . . . . . . . . . . . . . . 64

3.29. Images showing details of the displacement transducers that were installed at the bridge site: (a) different displacement transducers used; (b) measurement scheme used to take measurements in three directions. . . . . . . 65

3.30. Location of the crackmeters installed in the sides of the longitudinal girders. 66

3.31. Location of the crackmeters installed in the deck slab. . . . . . . . . . . 66

3.32. Strain transducers installed in section S1. . . . . . . . . . . . . . . 67

3.33. Details showing the installation of the strain transducers in the reinforcement (a); and, on the concrete surface (b) . . . . . . . . . . . 67

3.34. Arrangement of the data acquisition systems used in the long-term monitoring of the Jaguari River Bridge. . . . . . . . . . . . . . . . . . . 69

3.35. Web interface for data visualization of the vibrating wire sensor data. . . 70

3.36. Java based web interface for visualization of the recorded live-load data. . . 71

3.37. Histograms of the maximum recorded strain for live-loading events in the bottom reinforcement of the girders. . . . . . . . . . . . . . . . . 72

3.38. Histograms of the maximum recorded strain for live-loading events in the bottom reinforcement of the deck slab. . . . . . . . . . . . . . 73

3.39. Histograms and probability density plots comparing results for short and long-term monitoring of bottom reinforcement of inner girder in section S1. 74 
3.40. Histograms and probability density plots comparing results for short and long-term monitoring of bottom reinforcement of outer girder in section S1. 75

3.41. Histograms and probability density plots comparing results for short and long-term monitoring of bottom reinforcement of inner girder in section S3. 75

3.42. Histograms and probability density plots comparing results for short and long-term monitoring of bottom reinforcement of outer girder in section S3. 76

3.43. Histograms and probability density plots comparing results for short and long-term monitoring of bottom reinforcement of inner girder in section S5. 76

3.44. Histograms and probability density plots comparing results for short and long-term monitoring of bottom reinforcement of outer girder in section S5. 77

3.45. Histograms and probability density plots comparing results for short and long-term monitoring of transverse bottom reinforcement of deck slab. . . . 77

4.1. Mean and standard deviation of the number of daily loading events sorted by: (a) day of the week; and (b) month of the year.

4.2. Plots showing the behavior of the mean and standard deviation of the means and standard deviations of the maximum strain per loading event relative to: (a) day of the week and; (b) month of the year. . . . . . . . . . 8

4.3. Mean and standard deviation of the number of daily loading events sorted by: (a) day of the week; and (b) month of the year. . . . . . . . . . . . . 87

4.4. Probability density plots (solid lines) of the Gumbel MLE, for the different timeframes considered, and, respective, shifted yearly maximum distribution (dashed lines). . . . . . . . . . . . . . . . .

4.5. Probability density plots (solid lines) of the Gumbel MLE for the censored data (only business days), for the different timeframes considered, and, respective, shifted yearly maximum distribution (dashed lines) . . . . . . . . 90

4.6. Rolling mean and standard deviation of the collected data samples; in these plots, the parameters are calculated for an increasing number of samples to check convergence.

4.7. Rolling mean and standard deviation for the censored data (weekends and holidays removed). . . . . . . . . . . . . . . . . .

4.8. Kernel density estimation of the maximum strain for the registered loading events for strain gauge E16.

4.9. Transformed 1-day EVD showing the empirical results, the exact transformation from the kernel density estimate, and the corresponding fitted Gumbel distribution for strain gauge E16.

4.10. Resulting PDF's and CDF's for yearly maximum strain distribution obtained by transforming from a Gumbel fit of the transformed underlying distribution and fit to the daily maximum data. . . . . . . . . . 
4.11. Reinforcement distribution for the longitudinal girder at Section S5. . . . . 96

4.12. Simulated corrosion initiation time along with the fitted PDF. . . . . . . . 98

4.13. Plots showing: (a) annual reliability indices; (b) time-dependent reliability indices. . . . . . . . . . . . . . . . . . . . . . 9 98

A.1. Recorded strains in the static load test in section S1. . . . . . . . . . . 115

A.2. Recorded strains in the static load test in section $\mathrm{S} 2$. . . . . . . . . . . 116

A.3. Recorded strains in the static load test in section S3. . . . . . . . . . . . 117

A.4. Recorded strains in the static load test in section S5. . . . . . . . . . . 118

A.5. Recorded strains in the static load test at the deck (transverse direction at section S3). . . . . . . . . . . . . . . . . . . . . 119

A.6. Temperatures and strains recorded at Section S1. . . . . . . . . . . 120

A.7. Temperatures and strains recorded at Section S3. . . . . . . . . . . 121

A.8. Temperatures and strains recorded at Section S5. . . . . . . . . . . . . 122

A.9. Temperatures and crack opening at the girders. . . . . . . . . . . . . . 123

A.10.Temperatures and slab crack meter displacements. . . . . . . . . . . . . . . 124

A.11.Temperatures and midspan displacements. . . . . . . . . . . . . . . . 125

A.12.Temperatures and vertical displacements at supports. . . . . . . . . . . . 126

A.13.Temperatures and longitudinal displacements at supports. . . . . . . . . . 126

A.14.Temperatures and transverse displacements at supports. . . . . . . . . . 127 


\section{List of Tables}

2.1. List of random variables representing the parameters for the ultimate bending moment model. . . . . . . . . . . . . . . . . . 33

2.2. Mean and coefficient of variation of the distribution parameters for the random variables obtained in the ten runs of the updating algorithm for the three considered cases. . . . . . . . . . . . . . . . . 37

2.3. Scenarios considered for updating using three points in time. . . . . . . 38

2.4. Mean values and coefficient of variation of the random variable parameters obtained in the ten runs of the updating procedure for the four considered scenarios. . . . . . . . . . . . . . . . . . . . 40

3.1. Concrete strength test results for fresh concrete samples collected . . . . . 46

3.2. Maximum and minimum recorded strains during the short-term monitoring. 61

4.1. Results of the MLE of Gumbel distribution for the 1, 3, 7, 14, and 30-day maximum strain, along with the, respective, transformation to the yearly maximum strain distribution. . . . . . . . . . . . . . . . . 88

4.2. Results of the MLE of Gumbel distribution for the 1, 3, 5, 10, and 20-day maximum strain for business days, along with the, respective, transformation to the yearly maximum strain distribution. . . . . . . . . . . . . 90

4.3. Random variable definitions for the life-cycle analysis of cross-section S5. . 97 


\section{Contents}

$\begin{array}{ll}\text { 1. Introduction } & 15\end{array}$

1.1. Motivation . . . . . . . . . . . . . . . . . . 15

1.2. Research Objectives and Scope . . . . . . . . . . . . . . 16

1.3. Document Outline . . . . . . . . . . . . . . . . . . 16

2. Updating Life-Cycle Behavior of Reinforced Concrete Girders Using Field $\begin{array}{ll}\text { Testing Data } & 18\end{array}$

2.1. Introduction . . . . . . . . . . . . . . . . . . 18

2.2. Behavioral Model for Reinforced Concrete Girders . . . . . . . . . . . . . . 19

2.2.1. Modeling Reinforcement Corrosion . . . . . . . . . . . . . . . . 23

2.3. Updating Using the Bayesian Statistical Theory . . . . . . . . . . . . . . . 24

2.3.1. Basic Concepts and General Formulation . . . . . . . . . . . . . . . 24

2.3.2. Bayesian Updating of Mechanical Models . . . . . . . . . . . . . . . 26

2.3.3. Bayesian Updating Using Structural Reliability Methods . . . . . . 27

2.3.4. Bayesian Updating Using Subset Sampling . . . . . . . . . . . . . 28

2.3.5. Likelihood of Observations . . . . . . . . . . . . . . . . . . . . . . . 29

2.4. Updating Strength Prediction Using Field Testing Data . . . . . . . . . . . 30

2.4.1. Model Updating Using Data from a Single Test . . . . . . . . . . . 32

2.4.2. Model Updating Using Data from Multiple Tests . . . . . . . . . . 38

2.5. Final Considerations . . . . . . . . . . . . . . . . . . . . 41

3. Structural Health Monitoring of the Jaguari River Bridge 43

3.1. Description of the Structure . . . . . . . . . . . . . . . . 43

3.1.1. Location and Bridge Layout . . . . . . . . . . . . . . . . . . 43

3.1.2. Superstructure . . . . . . . . . . . . . . . . 43

3.1.3. Substructure . . . . . . . . . . . . . . . 45

3.1.4. Foundations and Abutments . . . . . . . . . . . . . . . . 45

3.1.5. Load Rating . . . . . . . . . . . . . . . . . . . . . . 46

3.2. Visual Inspection, Testing, and Short-Term Monitoring . . . . . . . . . . 47

3.2.1. Visual Inspections . . . . . . . . . . . . . . . . . . . . 47

3.2.1.1. Longitudinal Girders . . . . . . . . . . . . . . . . 47

3.2.1.2. Transverse Beams . . . . . . . . . . . . . . . . . . . 48 
3.2.1.3. Columns . . . . . . . . . . . . . . . . . . . 48

3.2.1.4. Deck Slab and Pavement . . . . . . . . . . . . . . . 48

3.2.1.5. Elastomeric Bearings . . . . . . . . . . . . . . . . . . . . . 49

3.2.2. Semi and Non-Destructive Testing . . . . . . . . . . . . . . . . . . 49

3.2.3. Bridge Field Testing and Short-Term Monitoring . . . . . . . . . . 50

3.2.3.1. Instrumentation . . . . . . . . . . . . . . 50

3.2.3.2. Short-Term Monitoring and Load Testing . . . . . . . . 56

3.2.4. Results from Short-Term Monitoring and Testing . . . . . . . . . 57

3.2.4.1. Static and Dynamic Testing . . . . . . . . . . . . 57

3.2.4.2. Short-Term Monitoring Results . . . . . . . . . . . . 59

3.3. Long-Term Monitoring System . . . . . . . . . . . . . . . . . . . . 62

3.3.1. Sensors . . . . . . . . . . . . . . . . . 63

3.3.2. Data Acquisition and Logging Systems . . . . . . . . . . . . . . . 68

3.3.3. Data Management and Visualization . . . . . . . . . . . . . 69

3.4. Overview of the Collected Data . . . . . . . . . . . . . . . . 70

3.4.1. Histograms of Live-Load Response . . . . . . . . . . . . . . . . . . 71

3.4.2. Comparison between Short-Term and Long-Term Monitoring . . . . 73

3.4.3. Vibrating Wire System . . . . . . . . . . . . . . . . . . . 78

3.5. Final Remarks . . . . . . . . . . . . . . . . . . . . . . 78

4. Probabilistic Life-Cycle Assessment Using Structural Health Monitoring 79

4.1. Introduction . . . . . . . . . . . . . . . . . . . . . 79

4.1.1. General Overview of the Literature . . . . . . . . . . . . . . . 79

4.2. Live-Load Effect Characterization Using SHM Data . . . . . . . . . . . . . 82

4.2.1. Concepts of the Extreme Value Theory . . . . . . . . . . . . . . . 82

4.2.1.1. Type I - Gumbel Distribution for Live-Load Data . . . . . 83

4.2.2. Application to the Jaguari River SHM Data . . . . . . . . . . . . . 85

4.2.2.1. Homogeneity of the Collected Live-Load Data . . . . . . . 85

4.2.2.2. Transformation assuming Gumbel Distribution . . . . . . 88

4.2.2.3. Transformation using Underlying Phenomenon Distribution 93

4.3. Life-Cycle Assessment of the Jaguari River Bridge Using SHM Data . . . . 96

4.4. Final Considerations . . . . . . . . . . . . . . . . . . . . . . . . 99

5. Conclusion and Future Works 101

5.1. Field Testing and Life-Cycle Assessment . . . . . . . . . . . . . . . . . . . 101

5.2. Structural Health Monitoring of the Jaguari River Bridge . . . . . . . . . . 102

5.3. Use of SHM Data in the Life-Cycle Assessment . . . . . . . . . . . . . . . 102

$\begin{array}{ll}\text { Bibliography } & 104\end{array}$ 
A. Collected Data from the Monitoring of the Jaguari River Bridge 114

A.1. Introduction . . . . . . . . . . . . . . . . . . . . . 114

A.2. Load-Test Results . . . . . . . . . . . . . . . . . . . . . . . . . . 114

A.3. Vibrating Wire Data . . . . . . . . . . . . . . . . . . 120 


\section{Introduction}

\subsection{Motivation}

The challenges that most developing and developed nations are facing in regard to its deteriorating infrastructure are well known. This issue is specially delicate for developing nations such as Brazil because, while it is important that investments be made to expand the existing infrastructure in order to support a growing economy, it is, at the same time, essential that investments be made in order to maintain its current infrastructure. Something that further aggravates this issue is the lack of a maintenance culture in some of these countries, which often leads to costly reactive maintenance practices. In this context, adequate state assessment and asset management techniques can considerably improve the decision making process.

In recent years, several novel approaches that apply probabilistic methods in the life-cycle management of civil infrastructure have been proposed [29, 44, 47, 66, 31, 71, 72, 77, 8]. These approaches use the reliability theory to create life-cycle models that integrate the uncertainties that are present in the problem and apply optimization techniques to obtain infrastructure management plans. An important aspect that must be taken into consideration in any infrastructure management system is how to incorporate newly obtained information. Towards this end, some frameworks have been proposed that optimize inspection and maintenance planing and integrate inspection data [43, 85]. These frameworks have the potential to be powerful tools for the cost-effective and rational decision making in the management of civil infrastructure. However, in order for the optimization frameworks to yield satisfactory results, it must be based on adequate life-cycle performance evaluation and prediction [28]. Therefore, attention must be given to the life-cycle performance assessment and prediction aspect of this field.

Techniques such as Structural Health Monitoring (SHM), Structural Testing, and Nondestructive Testing (NDT) have shown the potential of significantly improving the structural assessment of existing structures. The integration of data, obtained from these techniques, with life-cycle performance modeling is an interesting concept, because it allows for the improvement of these models by taking into consideration the observed behavior of the structure in the real world. Thus, an increasing amount of research effort has been focused on this area $[10,24,53,25,46,74,51,91,39,90]$. However, there are still relatively 
few successful cases of integration of field observations with life-cycle performance models, specially with data from structural testing and SHM. By taking into consideration these facts, it is clear that there is a strong need for further study of integration techniques of structural testing and SHM data into life-cycle performance analysis. This is further motivated by the data and experience obtained through two research projects involving structural testing and SHM of railway and highway bridges in which the author participated.

\subsection{Research Objectives and Scope}

The main objective of the present research is to investigate and develop procedures for integrating Structural Health Monitoring and structural testing data into life-cycle analysis and management of bridge structures. The specific goals of this research are listed below:

- Investigate monitoring and testing techniques used in the assessment of existing structures;

- Study of life-cycle reliability analysis and the performance metrics used in the integration with monitoring data of bridge structures;

- Development of frameworks for bridge structural life-cycle analysis with the inclusion of monitoring and testing data;

- Application of the frameworks to real-world problems.

Test results from railway bridges and data from a long-term monitoring system of a reinforced concrete highway bridge are used in this work. The collected data includes design and as-built drawings and documents, inspection documentation, measured strain and displacement data, and semi-destructive testing.

\subsection{Document Outline}

- Chapter 1 - Introduction: in this chapter a general introduction to the motivation and objectives of this research is presented, as well as the contents of this document.

- Chapter 2 - Updating Life-Cycle Behavior of Reinforced Concrete Girders Using Field Testing Data: the use of testing data to update parameters of a prediction model for reinforced concrete structures is explored in this chapter.

- Chapter 3 - Structural Health Monitoring of the Jaguari River Bridge: the installation and the characteristics of the monitoring system of the Jaguari River Bridge is shown in this chapter. 
- Chapter 4 - Probabilistic Life-Cycle Assessment Using Structural Health Monitoring: the integration of SHM data in the life-cycle assessment of structures is studied in this chapter.

- Chapter 5 - Conclusions and Future Works: This chapter presents some final remarks and conclusions on the topics considered in this work.

- Appendix A - Collected Data from the Monitoring of the Jaguari River Bridge: this appendix shows some of the data collected during the monitoring of the Jaguari River Bridge. 


\section{Updating Life-Cycle Behavior of Reinforced Concrete Girders Using Field Testing Data}

\subsection{Introduction}

Proper structural assessment is a key aspect of modeling the life-cycle behavior of any structure. Among the countless approaches for assessing existing structures, field-testing has shown to be of significant importance. In particular, applications of field-testing to the structural assessment of bridges have shown that this technique can yield significant improvements in the understanding of actual structural behavior and, has therefore, become a generally accepted practice $[7,64,75]$. This methodology usually consists in capturing the structural response through the measured output of sensors while applying a controlled load that, for the case of bridges, is usually done using a test vehicle with known axle loads. The measured output is, in most cases, obtained using strain, displacement, rotation, acceleration sensors, or a combination of these sensors.

Statistical inference is the method by which properties of an underlying distribution can be determined through data analysis. There are two main approaches to this problem, the frequentist approach and the Bayesian approach. During the last few decades, the Bayesian approach has found wide acceptance in the scientific community and has been applied to a fairly large scope of areas. Its application to structural engineering problems includes, but are not limited to, areas such as: updating of structural models for dynamic behavior [10], fracture mechanics [81], long-term creep deformations in concrete [80], corrosion in reinforced concrete [24, 50], and updating of load models [101].

The use of a Bayesian framework to update the probabilistic model using observed information with the objective of improving accuracy in structural reliability assessment is another important tool for performance assessment. Data from non-destructive testing, visual inspections, and vibration testing have been applied in conjunction with Bayesian updating to improve the reliability assessment [24, 92, 39]. Reliability updating using field-testing data is also a topic that has been explored: most of the applications involve 
using the actual load distribution factors obtained from testing [68, 76, 98], the truncation of the resistance distribution [68], or the calculation of a conditional probability of failure given the survival of the testing event $[34,23,26]$. These approaches use the structural response to update the analytical model and obtain new load distributions factors and use the loading data to update the probabilistic information. However, the response data can also be used, in some cases, to update the probabilistic information [50].

In the case of reinforced concrete structures, it is a known fact that strength and stiffness are highly correlated, however, there is a considerable level of uncertainty on the correlation between these factors. Therefore it is necessary to adopt a probabilistic approach when modeling the behavior of these structures. Over the last two decades the use of the Bayesian approach towards structural model updating has shown significant progress $[10,96,11,99,17]$. However, most of the work done focused on using acceleration measurements and the resulting frequency spectrums for updating the structural models and detecting damage.

With this in mind, this work establishes a comprehensive structural model that takes into consideration the nonlinear behavior, characteristics of reinforced concrete, and the degradation mechanism, which, in this case, considers only reinforcement corrosion. This model is then updated considering in-site displacement measurements under a controlled load. A Bayesian updating method presented by [90], which allows for the use of structural reliability methods was used. Model updating is carried out considering the flexural stiffness information and also carbonation depth measurements, which are used to update the parameters of the degradation model. Another approach in which tests are carried out in 5 to 10-year intervals is also implemented to study the applicability of the model updating procedure to life-cycle problems.

\subsection{Behavioral Model for Reinforced Concrete Girders}

In order to be able to study the effects of inspection and testing data in the structural evaluation, it is necessary to have a behavioral model for the structure that is able to predict the flexural strength and flexibility of reinforced concrete girders. For this purpose, moment-curvature relationships were used to determine the ultimate bending capacity and to calculate the stiffness information to calculate displacements. The moment-curvature relationship $(M-\phi)$ is determined through sectional analysis using the material and section properties by applying a layer-by-layer approach. In this approach, the cross section geometry and the reinforcement is divided into layers, as illustrated in Fig. 2.1. The section forces are calculated by adding the individual forces of each of these layers, 
as shown in eq. (2.1) below

$$
\begin{gathered}
N=\sum_{i=1}^{n_{c}} h_{i} b_{i} \sigma_{c}\left(\varepsilon_{c_{i}}\right)+\sum_{i=1}^{n_{s}} A s_{i} \sigma_{s}\left(\varepsilon_{s_{i}}\right) \\
M=\sum_{i=1}^{n_{c}}\left\{\left[h_{i} b_{i} \sigma_{c}\left(\varepsilon_{c_{i}}\right)\right]\left(y_{i}-y_{c g}\right)\right\}+\sum_{i=1}^{n_{s}}\left\{\left[A s_{i} \sigma_{s}\left(\varepsilon_{s_{i}}\right)\right]\left(y_{s_{i}}-y_{c g}\right)\right\}
\end{gathered}
$$

where, $h_{i}$ and $b_{i}$ are, respectively, the heights and widths of the individual cross section layers (fig. 2.1), $A s_{j}$ is the area of each reinforcement layer, $\sigma_{c}(\cdot)$ and $\sigma_{s}(\cdot)$ are the stresses obtained with constitutive models for the concrete and steel, respectively, $\varepsilon_{c_{i}}$ and $\varepsilon_{s_{j}}$ are, respectively, the strains in the concrete and steel layers, $y_{i}$ and $y_{s i}$ are, respectively, the depths of the cross section and reinforcement layers (Fig.2.1), $y_{c g}$ is the depth of the center of gravity of the cross section (fig. 2.1), and $n_{c}$ and $n_{s}$ are the number of cross section and reinforcement layers, respectively.

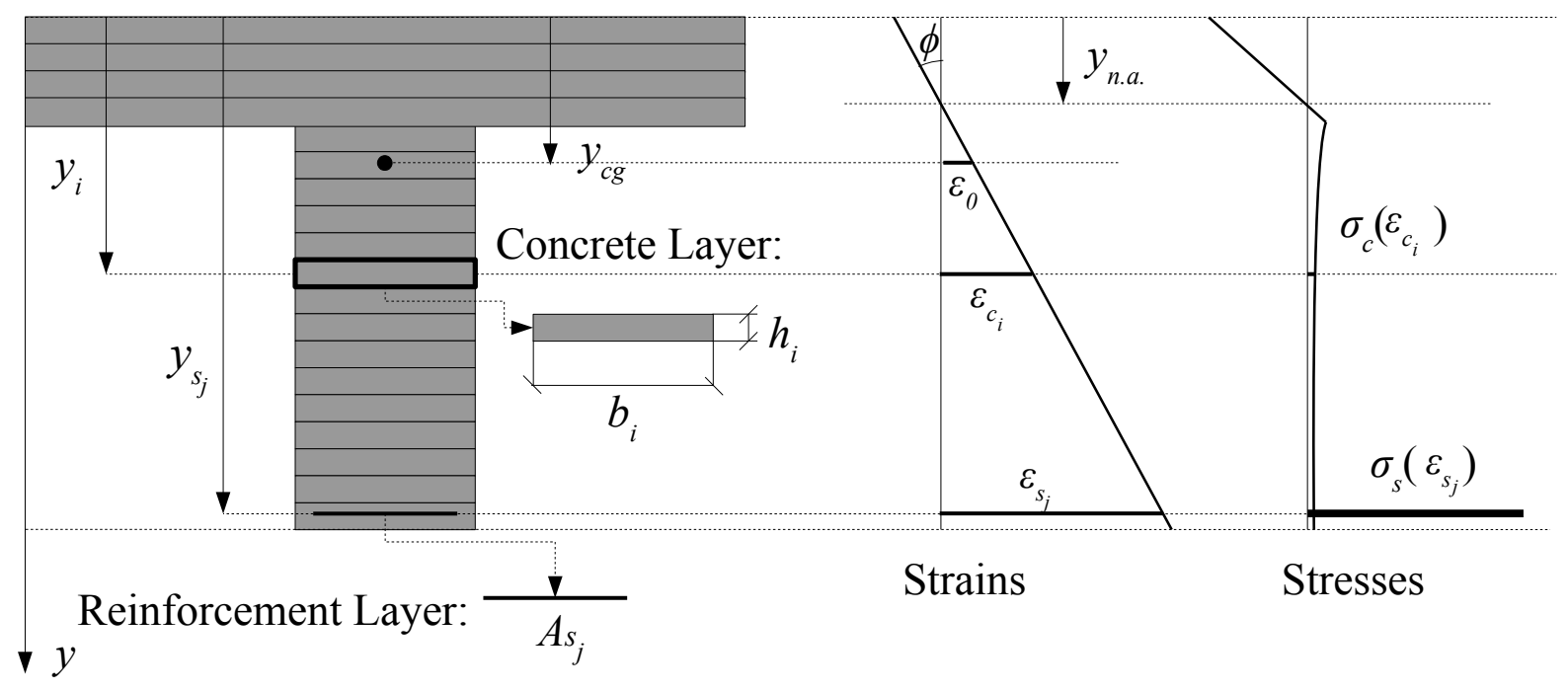

Figure 2.1.: Illustration of the layer-by-layer discretization of $\mathrm{RC}$ cross section including the strain and, corresponding stress field across the section depth.

Trough the use of adequate constitutive models for the materials, the stresses in each layer can be determined given a strain profile for the cross section. In this case, the hypothesis that a plane section remains plane during flexure is assumed. This approach allows for the characterization of the strain profile in the cross section by two variables which define a linear strain profile running trough the cross section height. For convenience these two variables were chosen as the strain in the center of gravity of the cross section, $\varepsilon_{0}$, and the curvature, $\phi$, which allows for determining the strain at each layer by using eq. (2.2).

$$
\begin{gathered}
\varepsilon_{c_{i}}=\varepsilon_{0}+\phi\left(y_{i}-y_{c g}\right) \\
\varepsilon_{s_{i}}=\varepsilon_{0}+\phi\left(y_{s_{i}}-y_{c g}\right)
\end{gathered}
$$


An elastic-plastic stress-strain curve was used for the reinforcement layers:

$$
\sigma_{s}(\varepsilon)= \begin{cases}-f_{s y} & \varepsilon<-\varepsilon_{s y} \\ E_{s} \varepsilon & -\varepsilon_{s y} \leq \varepsilon \leq \varepsilon_{s y} \\ f_{s y} & \varepsilon>\varepsilon_{s y}\end{cases}
$$

where, $f_{s y}$ and $E_{s}$ are the yield strain and the Young's modulus of the steel, respectively, and $\varepsilon_{s y}=f_{s y} / E_{s}$.

For the compression behavior of the concrete the stress-strain curved adopted in the fib Model Code 2010 [15] was used:

$$
\sigma_{c, \text { comp }}(\varepsilon)=-f_{c m} \frac{k \eta-\eta^{2}}{1+(k-2) \eta} \quad \varepsilon_{c, l i m}<\varepsilon<0
$$

where, $\eta=\frac{\varepsilon}{\varepsilon_{c 1}}$, the plasticity number $k=\frac{E_{c i}}{E_{c 1}}, \varepsilon_{c 1}$ is the strain at peak stress given by eq. (2.5), $E_{c i}$ is the modulus of elasticity of concrete at age of 28 days given by eq. (2.6), and $E_{c 1}$ is the secant stiffness at the peak stress (see Fig. (2.2)). In the equations below, $E_{c 0}$ is usually taken as $20.5 \times 10^{3} \mathrm{MPa}$ and $\alpha_{E}$ has the value of 1.0 for quartzitic aggregates (for other types of aggregates one may refer to Table 5.1-6 in the Model Code).

$$
\begin{aligned}
& \varepsilon_{c 1}=\frac{-1.60\left(f_{c m} / 10\right)^{0.25}}{1000} \\
& E_{c i}=E_{c 0} \alpha_{E}\left(\frac{f_{c m}}{10}\right)^{1 / 3}
\end{aligned}
$$

As shown in Fig. (2.2), the tensile behavior of concrete was separated into two parts, pre and post-cracking. As shown in eq. (2.7), the concrete is assumed to have linear behavior up to cracking and tension stiffening effects after cracking. This tension stiffening equation was originally proposed by Vecchio and Collins [97] and later adapted by Collins and Mitchell [18].

$$
\sigma_{c, t e n s}(\varepsilon)= \begin{cases}E_{c i} \varepsilon & 0<\varepsilon \leq \varepsilon_{c t} \\ \frac{\alpha_{1} \alpha_{2} f_{c t}}{1+\sqrt{500 \varepsilon}} & \varepsilon>\varepsilon_{c t}\end{cases}
$$



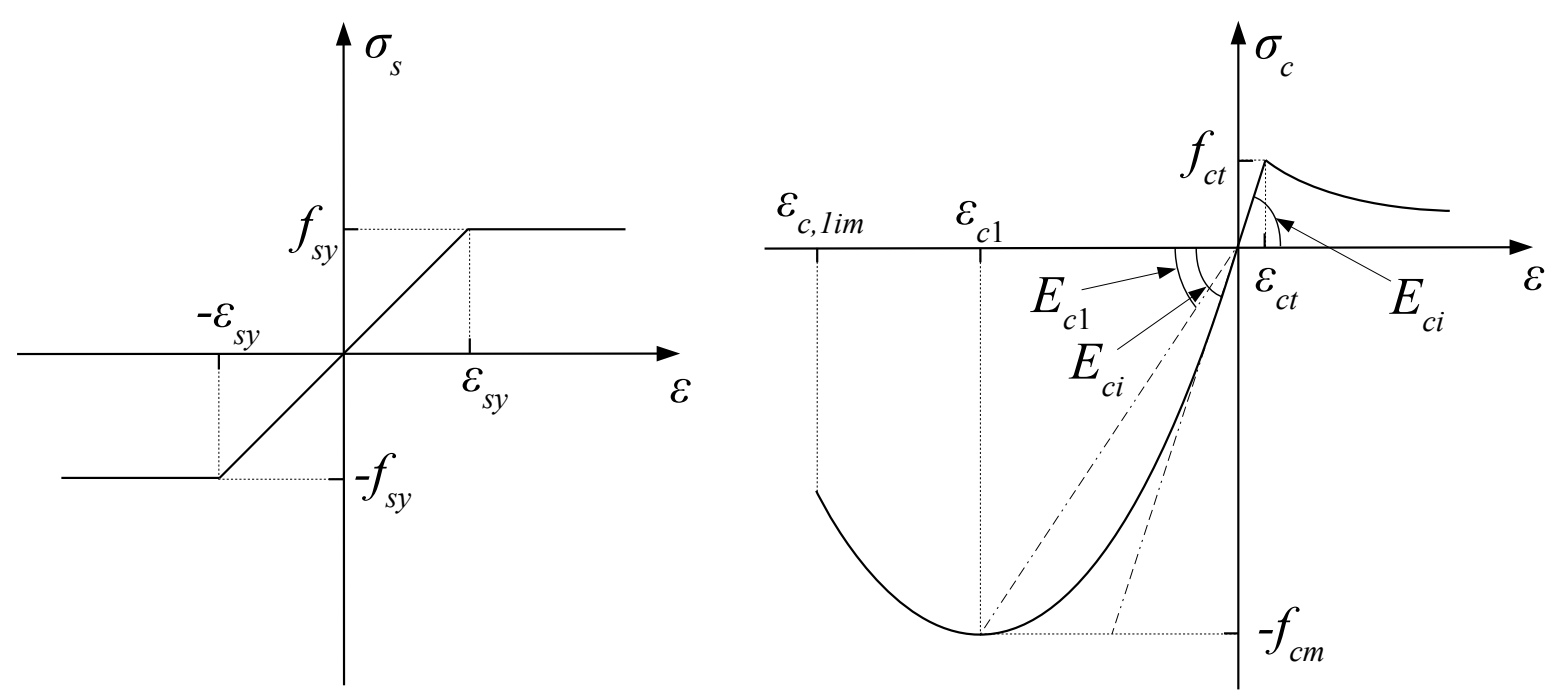

Figure 2.2.: Stress-strain curves for steel (left) and for concrete (right).

In the above equation, the factor $\alpha_{1}$ accounts for the bond characteristics of the reinforcement (1.0 for deformed bars, 0.7 for plain bars, wires, or bonded strands, and 0.0 for unbonded reinforcement) and $\alpha_{2}$ accounts for the loading condition (1.0 for short-term monotonic loading and 0.7 for permanent or repeated loads). For the tensile strength of concrete, $f_{c t}$, the following equation was adopted:

$$
f_{c t}=f_{\text {ctmo }}\left(\frac{f_{c m}}{10}\right)^{0.56}
$$

where, $f_{\text {ctmo }}=1.61 \mathrm{MPa}$.

Using the equations above it is possible to determine, for any strain field (characterized by $\phi$ and $\varepsilon_{0}$ ) on the cross-section, the corresponding acting forces $N$ and $M$. For the case of beams subjected to flexure without normal forces, a $M-\phi$ relationship can be obtained for this situation by setting $\phi$ values and determining the $\varepsilon_{0}$ value which yields $N=0$. The maximum value from this relationship is the ultimate bending moment, $M_{u}$, for this cross section.

Stiffness information for the beam can also be obtained from the $M-\phi$ relationship, considering the equation below:

$$
\frac{d^{2} v(s)}{d s^{2}}=-\phi=-\frac{M(s)}{E I(s)}
$$

where, $v(s)$ is the function that describes the beam displacements, $s$ is the distance along the longitudinal axis of the beam, $M(s)$ is the bending moment acting on the beam, 
and, $E I(s)$ is the flexural stiffness parameters for the beam. It is possible to obtain from the $M-\phi$ relationship the stiffness parameter values, $E I(s)$, that correspond to $M(s)$. These stiffness values can be used in an analytical model to compute the displacements considering the nonlinear behavior of concrete.

\subsubsection{Modeling Reinforcement Corrosion}

An essential aspect of life-cycle analysis lies in determining the degradation mechanisms that are present. Although there are many causes of degradation in reinforced concrete structures, reinforcement corrosion is considered as being one of the most critical ones $[84,63,100]$. Therefore, in this study, the effects of this type of damage on the response of these structures is considered.

Corrosion initiates due to the depassivation of the reinforcement within the concrete which can, typically, be induced by either carbonation or chlorides. In the first case, the carbon dioxide, present in the atmosphere, reacts with the concrete, reducing its alkalinity. Once the carbonation depth reaches the reinforcement it destroys the protective oxide layer of the reinforcement and corrosion is initiated. The second case occurs due to chloride ions, generally present in the atmosphere in costal regions or in the de-icing salts used in cold regions. These ions penetrate the concrete by diffusion and, once a critical concentration is reached at the reinforcement depth, depassivation occurs.

In the case study presented in this work only carbonation induced corrosion was considered since there was no significant presence of chloride ions. Therefore, the reinforcement corrosion model shown here will only take into consideration initiation due to carbonation. However the methodology shown here can be easily adapted to chloride induced corrosion.

The progress of the carbonation front may be modeled as a diffusion process of carbon dioxide through the concrete as shown in eq. 2.10, where $x_{c}(t)$ is the carbonation depth, $t$ is the time (usually in years), and $K$ is the diffusion coefficient (also called carbonation constant throughout this thesis).

$$
x_{c}(t)=K \sqrt{t}
$$

An inherent hypothesis, in this equation, is that the diffusion coefficient is a constant material property. As can be seen, for example, in the fib Model Code for Service Life Design [37], the carbon dioxide diffusion process depends on several environmental and material characteristics, which may change during the structure's lifetime. However this equation allows for a simple estimation of the corrosion initiation time and, when used with in-situ carbonation depth measurements, can yield reliable results. 
Once the carbonation depth reaches the reinforcement, the corrosion propagation stage starts. For this work, uniform corrosion of the rebar was considered [93, 30] and the rebar area was calculated using eq. 2.11 , where $D_{b}$ is the rebar diameter, $t_{i}$ is the initiation time, and $\nu$ is the corrosion rate. In the implementation of the layer-by-layer discretization, adopted here, the rebars are separated into groups with the same cover and depth. The corrosion initiation time, $t_{i}$, for each group can be determined using eq. 2.10 based on the groups concrete cover.

$$
A_{s}(t)= \begin{cases}\frac{\pi D_{b}^{2}}{4} & t \leq t_{i} \\ \frac{\pi}{4}\left[D_{b}-2 \nu\left(t-t_{i}\right)\right] & t>t_{i}\end{cases}
$$

Using the layered cross section model associated with a time-dependent reinforcement area it is possible to estimate the lifetime behavior of the reinforced concrete girder. In the following sections a procedure is shown in which some of the model parameters will be considered as random variables. Testing data is then used to update these variables and improve the prediction model.

\subsection{Updating Using the Bayesian Statistical Theory}

\subsubsection{Basic Concepts and General Formulation}

In the Bayesian approach to parameter estimation, the parameters themselves are treated as random variables, which is one of the main differences between this and the frequentist approach. To demonstrate its basic principles, consider an event $A$ and a set of mutually exclusive and collectively exhaustive events $\theta_{1}, \theta_{2}, \ldots, \theta_{n}$, regarding these events, Bayes' theorem in its general form states the following.

$$
P\left(\theta_{i} \mid A\right)=\frac{P\left(\theta_{i}\right) P\left(A \mid \theta_{i}\right)}{\sum_{j=1}^{n} P\left(A \mid \theta_{j}\right) P\left(\theta_{j}\right)} .
$$

The event of interest is $\theta_{i}$ and $P\left(\theta_{i}\right)$ is the prior knowledge for its occurrence probability. The probability of event $\theta_{i}$ occurring given that event $A$ has been observed can be interpreted as the posterior probability of occurrence of $\theta_{i}, P\left(\theta_{i} \mid A\right)$. Also in this case, the term $P\left(A \mid \theta_{i}\right)$ can be interpreted as the likelihood of event $A$ being observed given that event $\theta_{i}$ has occurred. In practical terms, the set of events $\theta_{i}$ can represent a set 
of hypotheses, in which case $P\left(\theta_{i}\right)$ will represent the prior knowledge about the probability of hypothesis $\theta_{i}$ being true, $P\left(\theta_{i} \mid A\right)$ the posterior knowledge about hypothesis $\theta_{i}$ given that $\mathrm{A}$ has been observed, and $P\left(A \mid \theta_{i}\right)$ the likelihood of observing $A$ given that hypothesis $\theta_{i}$ is true.

When dealing with parameter estimation, this set of hypotheses, $\theta_{i}$, can be possible values of a parameter, $\theta$, that one wishes to estimate. In this case, considering a continuum of possible values for $\theta$, as opposed to a set of discrete values, is very practical. Assuming that the random variable $\Theta$ represents this parameter, $\theta$ which is to be estimated, with a prior PDF $f^{\prime}(\theta)$, the posterior distribution, $f^{\prime \prime}(\theta)$, after observing $A$ can be obtained by equation $2.13[5]$.

$$
f^{\prime \prime}(\theta)=\frac{f^{\prime}(\theta) P(A \mid \theta)}{\int_{-\infty}^{\infty} P(A \mid \theta) f^{\prime}(\theta) d \theta}
$$

Equation 2.13 is usually also expressed as,

$$
f^{\prime \prime}(\theta)=k L(\theta) f^{\prime}(\theta)
$$

in which $L(\theta)=P(A \mid \theta)$ is the likelihood function that represents the probability of observing $A$ given the parameter value $\theta$ and $k=\frac{1}{\int_{-\infty}^{\infty} L(\theta) f^{\prime}(\theta) d \theta}$ is the normalizing constant. The expected value of $\theta$ can be used as a point estimator of the parameter and can be obtained through equation 2.15 [5].

$$
\hat{\theta}^{\prime \prime}=E(\Theta \mid A)=\int_{-\infty}^{\infty} \theta f^{\prime \prime}(\theta) d \theta
$$

If the observed outcome $A$ is substituted for a set of observations $\left(x_{1}, x_{2}, \ldots, x_{n}\right)$ from the population of the underling random variable $X$, equation 2.14 can be generalized as[5],

$$
f^{\prime \prime}(\theta)=k L(\theta) f^{\prime}(\theta)
$$

with $k=\frac{1}{\int_{-\infty}^{\infty}\left(\prod_{i}^{n} f_{X}\left(x_{i} \mid \theta\right)\right) f^{\prime}(\theta) d \theta}$ and $L(\theta)=\prod_{i=1}^{n} f_{X}\left(x_{i} \mid \theta\right)$.

It is possible to obtain significant mathematical simplifications to equations (2.14) and (2.16) based on the appropriate choice of the probability distribution of the parameter 
based on the type of distribution of the underlying variable random variable. This pairing is sometimes called conjugate pairs or conjugate distributions, a collection of these can be found in [27]. For the cases where no closed form can be obtained, there exists numerical alternatives, such as Markov chain Monte Carlo, Metropolis-Hastings algorithm, and slice sampling algorithm, that allow for sampling of the posterior distribution. These methods are of interest since they do not require the calculation of the integral in the normalizing constant $k$ which can be computationally difficult and time consuming.

The formulation above shows that the Bayes' theorem can be interpreted as a formula for obtaining the posterior distribution from a prior distribution using observed information and therefore is the basis for any Bayesian updating procedure. When direct observations from the underlying random variable are available, equation (2.16) can be applied directly in the updating process.

Bayesian updating can also be carried out in cases where the data is not directly available from the random variables themselves, but from a property that can be calculated through a prediction model that is dependent on these random variables. This updating methodology will be presented in the following subsection.

\subsubsection{Bayesian Updating of Mechanical Models}

In Bayesian updating of mechanical models, the model parameters are considered as random variables and the initial distribution can be updated given the observed data through the Bayes' theorem. Therefore, considering the random variables $\mathbf{X}$ representing the model parameters with a prior distribution $f^{\prime}(\mathbf{x})$ and a set of observed data $\boldsymbol{\theta}$, the posterior distribution for the random variables can be obtained through eq. (2.17).

$$
f^{\prime \prime}(\mathbf{x})=\frac{L(\boldsymbol{\theta} \mid \mathbf{X}=\mathbf{x}) f^{\prime}(\mathbf{x})}{\int_{\mathbf{X}} L(\boldsymbol{\theta} \mid \mathbf{X}=\mathbf{x}) f^{\prime}(\mathbf{x}) d \mathbf{x}}
$$

Where $L(\boldsymbol{\theta} \mid \mathbf{X}=\mathbf{x})$ is called the likelihood function and is associated with the probability of occurrence of the observed data $\boldsymbol{\theta}$ given $\mathbf{x}$. For simplicity, this function is commonly represented as $L(\mathbf{x})$.

In most practical applications it is difficult to use eq. (2.17) due to difficulties in calculating the multidimensional integral. However, using the direct proportionality between the posterior, $f^{\prime \prime}(\mathbf{x})$, and $L(\mathbf{x}) f^{\prime}(\mathbf{x})$, sampling methods can be applied to determine the posterior. For this purpose Markov Chain Monte Carlo methods have been successfully used in some applications $[9,81,17]$. In this work, a Bayesian updating procedure presented by [90] that allows for the use of structural reliability methods was used. 


\subsubsection{Bayesian Updating Using Structural Reliability Methods}

This method is composed on considering an augmented space consisting of the random variables $\mathbf{X}$ and $P$, where $\mathbf{X}$ is the random variables for the model parameters and $P$ is a standard uniform variable in $[0,1]$. In this augmented space $[\mathbf{x}, p]$ the following domain can be defined using the likelihood function

$$
\Omega=[p \leq c L(\mathbf{x})]
$$

where $\mathrm{c}$ is a constant that guarantees that $c L(\mathbf{x}) \leq 1$ for all $\mathbf{x}$. This domain describes the observed event(s) in the augmented space.

It can be demonstrated (see [90]) that the posterior distribution in eq. (2.17) can be rewritten as eq. (2.19).

$$
f^{\prime \prime}(\mathbf{x})=\frac{\int_{p \in \Omega} f^{\prime}(\mathbf{x}) d p}{\int_{[\mathbf{x}, p] \in \Omega} f^{\prime}(\mathbf{x}) d p d \mathbf{x}}
$$

The denominator in eq. (2.19) corresponds to a structural reliability problem with the domain $\Omega$ being the event of interest. From this equation it follows that the samples generated from the prior distribution, $f^{\prime}(\mathbf{x})$, that fall into the domain $\Omega$ are distributed according to the posterior distribution $f^{\prime \prime}(\mathbf{x})$. A simple rejection sampling algorithm may be used to sample from $f^{\prime \prime}(\mathbf{x})$. However, as the amount of new information increases, and the likelihood function values decreases, the use of rejection sampling becomes inefficient. Through the use of structural reliability methods it is possible to increase the acceptance rate of the sampling.

When dealing with structural reliability problems it is often useful to transform the original augmented space of $\mathbf{X} \in \mathbb{R}^{n}$ and $P \in \mathbb{R}$ into a space with independent standard normal variables $\mathbf{Z}=\left[\begin{array}{llll}Z_{0} ; & Z_{1} ; \ldots & \ldots Z_{n}\end{array}\right] \in \mathbb{R}^{n+1}$. The transformation of $\mathbf{X}$ and $P$ into $\mathbf{Z}$ can be done separately due to their independence. Usual transformation methods that are used in structural reliability, such as the Nataf or the Rosenblatt[56], can be used to transform $\mathbf{X}$ as shown in eq. (2.20)

$$
X=T\left(\left[Z_{1}, Z_{2}, \ldots, Z_{n}\right]\right)
$$

where, $\mathrm{T}$ is the transformation method adopted. The transformation of $\mathrm{P}$ into $\mathrm{Z}$ is done 
using the standard normal cumulative distribution function (CDF), $\Phi$, as shown in eq. $(2.21)$.

$$
P=\Phi\left(Z_{0}\right)
$$

Using these transformations it is possible to obtain $\Omega_{Z}$ which is equivalent to the domain $\Omega$ in the space of the variables $\mathbf{Z}$.

$$
\Omega_{Z}=\left\{\Phi\left(Z_{0}\right) \leq c L\left[T\left(Z_{1}, \ldots Z_{n}\right)\right]\right\}
$$

The observation domain can be stated in terms of the limit state function presented in eq. (2.23) so that $\Omega_{Z}=\{H(z) \leq 0\}$.

$$
H(z)=z_{0}-\Phi^{-1}\left\{c L\left[T\left(z_{1}, \ldots, z_{n}\right)\right]\right\}
$$

Thus by determining the points that fall into the failure domain, defined by this limit state equation, and transforming these points back to the original space $\mathbf{X}$, one obtains samples from the posterior distribution. Many structural reliability methods can be applied for this purpose with varying degrees of success based on their capability of sampling adequately from the failure domain. Among the available methods, the subset sampling method [6] has shown to be adequate in evaluating small failure probabilities. The application of this method with Bayesian updating is shown in the next section.

\subsubsection{Bayesian Updating Using Subset Sampling}

In the subset sampling method proposed by [6], the calculation of a low probability event is substituted by the calculation of a series of more probable events. Assuming that $F$ represents a failure event and its corresponding failure region, it is possible to define a decreasing sequence of failure events that are nested $F_{1} \supset F_{2} \supset \ldots \supset F_{m}$ in which $F_{m}=F$. The probability of occurrence of event $F, P(F)$ can then be expressed in terms of these intermediate failure events, as shown in eq.(2.24), by calculating $P\left(F_{1}\right)$ and the sequence of conditional probabilities $P\left(F_{i+1} \mid F_{i}\right), i=1, \ldots, m-1$.

$$
P(F)=P\left(F_{m}\right)=P\left(\bigcap_{i=1}^{m} F_{i}\right)=P\left(F_{1}\right) \cdot \prod_{i=1}^{m-1} P\left(F_{i+1} \mid F_{i}\right)
$$


The simulation method consists on using Monte Carlo Simulation (MCS) to determine $P\left(F_{1}\right)$, following this step, a Markov chain MCS is used to obtain samples that fall into the region $F_{1}$, with these samples $P\left(F_{2} \mid F_{1}\right)$ is calculated. During each step of the process, samples are taken from the region $F_{i}$ to estimate $P\left(F_{i+1} \mid F_{i}\right)$, this process is repeated until $P\left(F_{m} \mid F_{m-1}\right)$ is obtained and $P(F)$ can be calculated. For each step, the samples that fall into the next intermediate event region are used as seeds to the Markov chain MCS of the following step, which makes the algorithm more efficient since this Markov chain does not require the burn-in period[102, 79]. Here the modified Metropolis algorithm originally presented by [6] was used.

Considering the limit state equation for the observation data given by eq. (2.23), the intermediate events regions can be defined as $F_{i}=\left\{H(z) \leq y_{i}\right\}$, where $i=1, \ldots, m$ and $y_{1}>y_{2}>\ldots>y_{m}=0$. The values of $y_{i}$ are chosen adaptively so that the conditional probability $P\left(F_{i+1} \mid F_{i}\right)$ in each step is approximately $p_{0}$ as suggested by [6].

Essentially the same algorithm used for the calculation of the probability associated with the event of interest can be used for the updating problem. The exception being that, in the last step, instead of calculating the final probability, the samples from $F_{m-1}$ that fall into $F_{m}$ are used to sample from $F_{m}$ which is the domain of the observation data. By transforming these samples from the $\mathbf{Z}$ variable space to the $\mathbf{X}$ variable space, samples from the posterior $f^{\prime \prime}(x)$ are obtained.

\subsubsection{Likelihood of Observations}

In the preceding sections the Bayesian updating problem was formulated in terms of a structural reliability problem and a solution method was presented. This section presents some considerations regarding the definition of the likelihood of the observation.

Generally observations can be separated into two types: equality information and inequality information $[21,89]$. Observations that are made up of survival or failure events can be modeled through $H_{i}(\mathbf{x})$ in order to define the observation domain in eq. (2.25).

$$
\Omega_{i}=\left[\mathbf{x} \in \mathbb{R}^{n}: H_{i}(\mathbf{x}) \leq 0\right]
$$

For this type of observation $M_{i}(x)$ is, from the reliability point of view, a limit state function and the likelihood can be stated as

$$
L_{i}(\mathbf{x})=P\left(\Omega_{i} \mid \mathbf{X}=\mathbf{x}\right)=I\left[H_{i}(\mathbf{x}) \leq 0\right]
$$


where, $I$ is the indicator function.

When the type of observation is of the equality type, the likelihood function can be stated in terms of the probability density function, $f_{\varepsilon_{i}}$, of the error between the measurement, $d_{i}$, and the predicted result, $M_{i}(\mathbf{x})$. The error may be considered to follow the Normal distribution which, as mentioned in [96], is justified by the Principle of Maximum Entropy[38]. If this error is of the additive type, the likelihood function may be stated as in eq. (2.27); if, however, the error is of the multiplicative type, the likelihood can then be stated according to eq. (2.28).

$$
\begin{aligned}
& L_{i}(\mathbf{x})=f_{\varepsilon_{i}}\left[d_{i}-M_{i}(\mathbf{x})\right]=\frac{1}{\sigma_{\varepsilon, i} \sqrt{2 \pi}} \exp \left\{-\frac{1}{2 \sigma_{\varepsilon, i}^{2}}\left[\left(M_{i}(\mathbf{x})-d_{i}\right)-\mu_{\varepsilon, i}\right]^{2}\right\} \\
& L_{i}(\mathbf{x})=f_{\varepsilon_{i}}\left[\frac{d_{i}}{M_{i}(\mathbf{x})}\right]=\frac{1}{\sigma_{\varepsilon, i} \sqrt{2 \pi}} \exp \left[-\frac{1}{2 \sigma_{\varepsilon, i}^{2}}\left(\frac{d_{i}}{M_{i}(\mathbf{x})}-\mu_{\varepsilon, i}\right)^{2}\right]
\end{aligned}
$$

When a set of measurements is available and it may be assumed that each reading is statistically independent, a combined likelihood can determined trough eq. 2.29.

$$
L(\mathbf{x})=\prod_{i=1}^{m} L_{i}(\mathbf{x})
$$

\subsection{Updating Strength Prediction Using Field Testing Data}

To better illustrate the integration and effects of field testing data, a practical example will be used. The reinforced concrete railway bridge built in 1973, shown in Fig. 2.3, was studied in 2011 by a research team from the University of São Paulo. This structure was instrumented with strain gauges and displacement sensors to measure the in-service structural response. The rails near one of the abutments of the bridge were instrumented with strain gauges in a shear-bridge configuration that allowed for measuring the vertical forces applied by the train wheels on the rails. Results from concrete core testing and carbonation depth were also carried out during the field work.

As mentioned, in Bayesian Model Updating, the model parameters themselves are considered as random variables. Therefore the main parameters for the behavioral model of reinforced concrete girders shown previously were considered as random variables for 


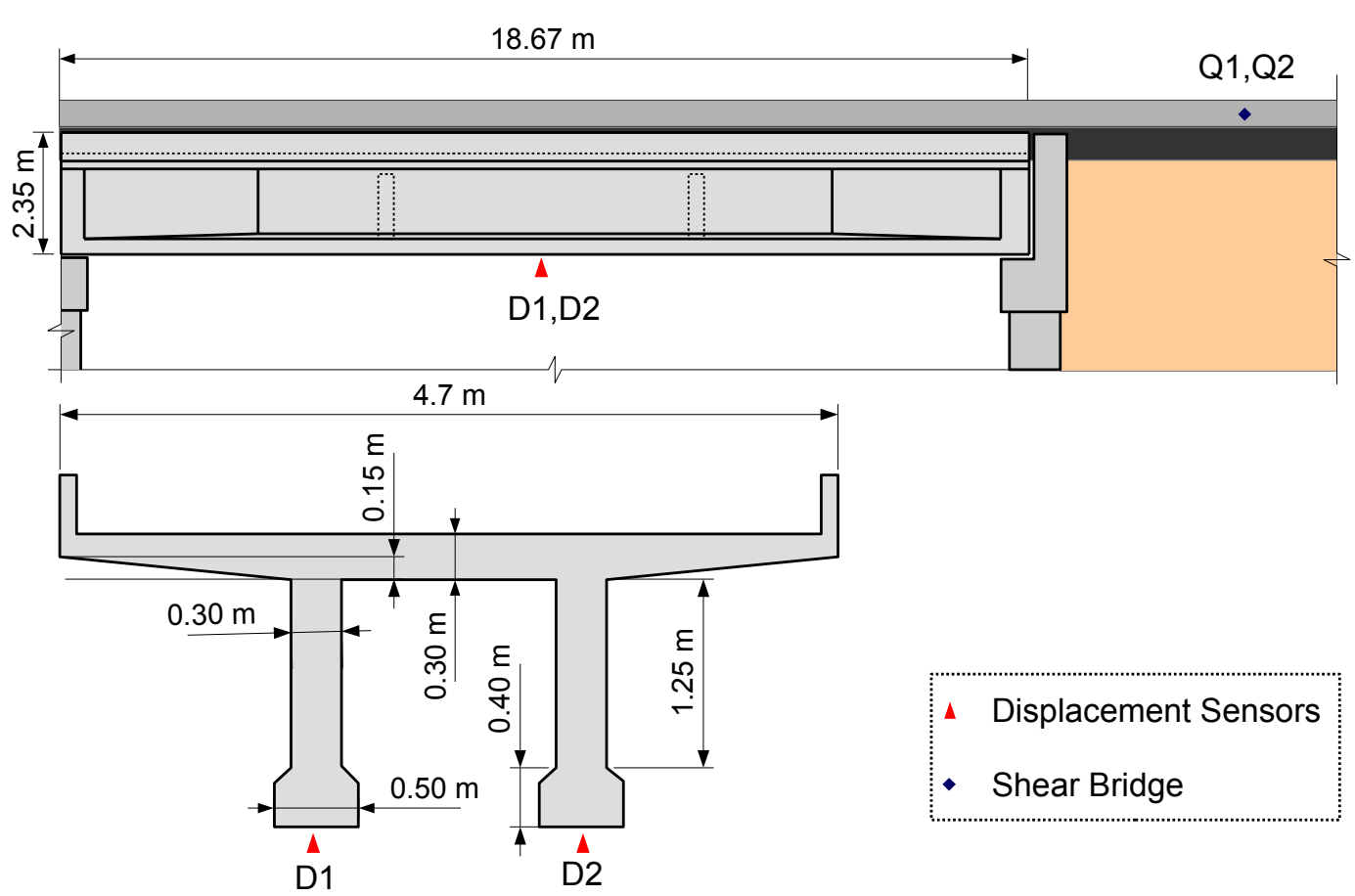

Figure 2.3.: Bridge geometry and sensor positioning.

the updating problem. The distributions associated with each of the parameters shown in Table 2.1 were determined by consulting some of the relevant references in this field $[62,60,61,69,30]$. For the concrete strength, steel yield strength, and the concrete covers the mean values were based on the nominal values from the original design documents. Since the model considers the nonlinear behavior of concrete it is important to estimate the dead loads acting on the structure at the time of the test. The load contribution from the self-weight of the structure, ballast, track, and guard-rails and other accessories were quantified using the available design documents. Due to uncertainties involved in the estimation of the present dead loads an uncertainty factor, $\gamma_{D L}$, was adopted with the distribution parameters shown in Table 2.1, which were obtained through simulation by considering the variability of all load components. In the simulation, the measured live loads are applied together with the dead loads and the change in response due to the live loads is the output used in the updating procedure (since this was the measured response).

The data obtained from the shear-bridges installed in the rails was processed and the individual wheel loads were obtained (Figure 2.4). At the time of the test, the locomotive and wagon types were registered and, using their geometry information and the measured wheel loads, a train loading for the analysis model was determined. By applying this train loading with the estimated dead loads to the previously shown nonlinear analytical model one is able to obtain the predicted girder displacements. Since the shear bridges measure de force that the wheels apply to the rail, dynamic effects do not allow for the 


\subsection{Updating Strength Prediction Using Field Testing Data}

determination of the vehicle weight. However, in this case the train passage was done at low speed and, therefore, no dynamic effect were expected.

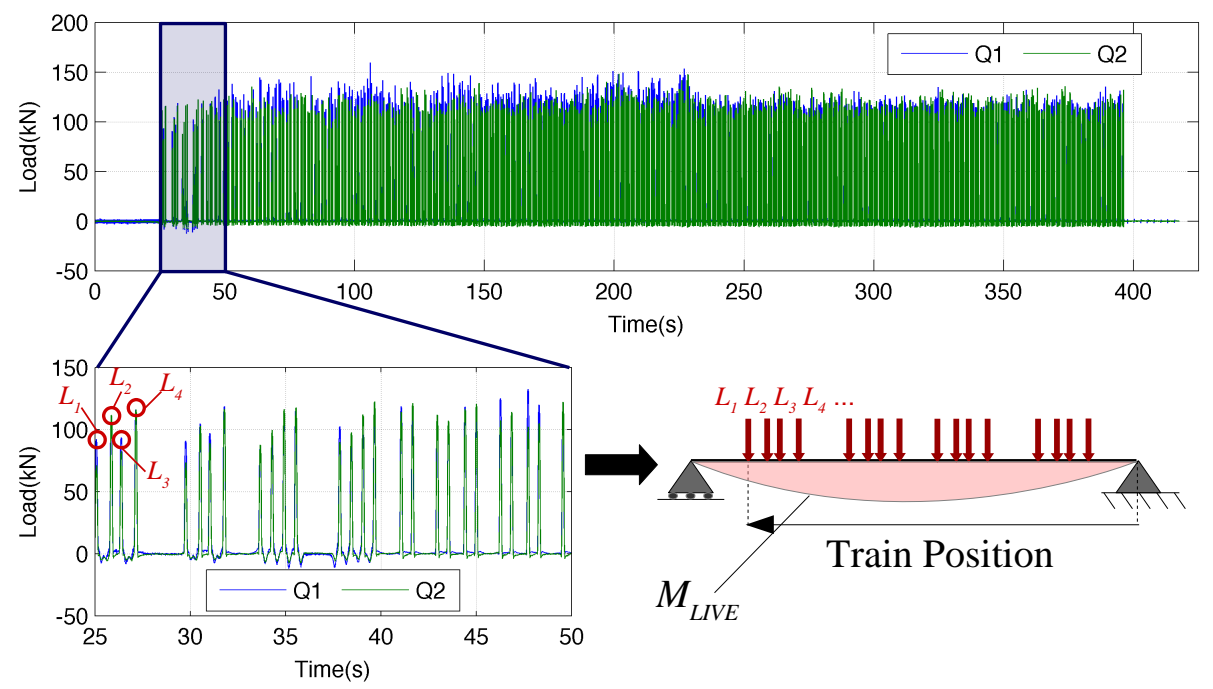

Figure 2.4.: Use of measured wheel loads to determine train loading on the bridge.

\subsubsection{Model Updating Using Data from a Single Test}

As shown in fig. 2.3, two displacement sensors were installed at the midspan of the bridge under each of the longitudinal girders. For simplicity, the analysis was carried out considering a single girder with the cross section shown in fig. 2.3. Therefore, the measured displacement, $d$, considered in the analysis, is the average reading between the two displacement sensors and the individual wheel loads were summed to obtain the axle loads to be applied to the model. For this specific case, the error was considered as multiplicative with c.o.v. of $10 \%$, and a bias factor of 0.85 was also considered in the error function. This bias factor was adopted because the structure may present increased stiffness due to rotational stiffness in the bearings and contribution of the track which are effects that were not captured by the model. Not considering this bias factor can potentially lead to unconservative strength predictions in the case where the structure is stiffer because of factors that are not considered in the model parameters. The likelihood in eq. 2.28 was used since the error was considered as multiplicative.

A dataset consisting of 10 displacement values was used in the updating procedure by applying eq. 2.29 to calculate the combined likelihood of the observed data. It is relevant to mention that, as the number of observations increase the model analysis time increases and, also, the likelihood values tend to decrease rapidly. This decrease in the likelihood values leads to lower sample acceptance rates in the sampling algorithm which, consequently, yields higher computation times and lower sample quality in the output. 


\begin{tabular}{|c|c|c|c|c|c|}
\hline \multirow{4}{*}{$\begin{array}{c}\text { Model } \\
\text { Parameter } \\
f_{c}\end{array}$} & \multicolumn{3}{|c|}{ Distribution } & \multirow{2}{*}{ Unit } & \multirow{2}{*}{ Description } \\
\hline & Type & \multicolumn{2}{|c|}{ Parameters } & & \\
\hline & \multirow{2}{*}{ Lognormal } & 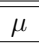 & 24.6 & \multirow{2}{*}{$\mathrm{MPa}$} & \multirow{2}{*}{ Concrete strength } \\
\hline & & $\sigma$ & 6 & & \\
\hline \multirow{2}{*}{$f_{\text {ctmo }}$} & \multirow{2}{*}{ Lognormal } & $\mu$ & 1.61 & \multirow{2}{*}{$\mathrm{MPa}$} & \multirow{2}{*}{ See eq. $(2.8)$} \\
\hline & & $\sigma$ & 0.322 & & \\
\hline \multirow{2}{*}{$E_{c 0}$} & \multirow{2}{*}{ Normal } & $\mu$ & $2.05 \times 10^{4}$ & \multirow{2}{*}{$\mathrm{MPa}$} & \multirow{2}{*}{ See eq. $(2.6)$} \\
\hline & & $\sigma$ & $1.64 \times 10^{3}$ & & \\
\hline \multirow{2}{*}{$f_{s y}$} & \multirow{2}{*}{ Lognormal } & $\mu$ & 560 & \multirow{2}{*}{$\mathrm{MPa}$} & \multirow{2}{*}{ Steel yield strength } \\
\hline & & $\sigma$ & 28.0 & & \\
\hline \multirow{2}{*}{$E_{s}$} & \multirow{2}{*}{ Normal } & $\mu$ & $2.1 \times 10^{5}$ & \multirow{2}{*}{$\mathrm{MPa}$} & \multirow{2}{*}{ Modulus of elasticity of steel } \\
\hline & & $\sigma$ & $6.93 \times 10^{3}$ & & \\
\hline \multirow{2}{*}{$A s / A s_{n o m}$} & \multirow{2}{*}{ Normal } & $\mu$ & 1.00 & \multirow{2}{*}{ - } & \multirow{2}{*}{ Reinforcement area } \\
\hline & & $\sigma$ & 0.025 & & \\
\hline \multirow{2}{*}{$c_{b o t}$} & \multirow{2}{*}{ Lognormal } & $\mu$ & 20 & \multirow{2}{*}{$\mathrm{mm}$} & \multirow{2}{*}{ Bottom reinforcement cover } \\
\hline & & $\sigma$ & 8 & & \\
\hline \multirow{2}{*}{$c_{\text {top }}$} & Loonormal & $\mu$ & 15 & $\mathrm{~mm}$ & Top reinforcement cover \\
\hline & & $\sigma$ & 10 & IIIIm & 10p remlorcement cover \\
\hline & Loonormal & $\mu$ & 15 & $\mathrm{~mm}$ & Lateral reinforcement cover \\
\hline Clat & Lognormal & $\sigma$ & 8 & IIIm & Lateral reminorcement cover \\
\hline$K$ & Lognormal & $\mu$ & 4 & $\mathrm{~mm} \cdot \mathrm{yr}^{-1 / 2}$ & Carbonation front constant \\
\hline & & $\sigma$ & 1.6 & & \\
\hline$\nu$ & Lognormal & $\mu$ & $8.9 \times 10^{-3}$ & $\mathrm{~cm} \cdot \mathrm{yr}^{-1}$ & Corrosion rate \\
\hline$\nu$ & Lughivilitial & $\sigma$ & $2.5 \times 10^{-3}$ & CII1 - y1 & Golusion late \\
\hline$\gamma_{D}$ & Normal & $\mu$ & 1.05 & - & Dead load uncertainty \\
\hline TDL & & $\sigma$ & 0.105 & & \\
\hline
\end{tabular}

Table 2.1.: List of random variables representing the parameters for the ultimate bending moment model.

The choice, made for this application, was based on a study of different number of observations which yielded satisfactory results with a reasonable processing time. Techniques to improve the acceptance rates when dealing with problems with higher number of observations have been the focus of recent studies $[12,90]$. However, these techniques were not tested in this application.

For consistency ten sequential runs of the updating algorithm were carried out, the resulting updated PDFs, along with the the prior PDFs, of these runs are shown in fig. 2.5. Significant changes in the parameters that influence the concrete tensile strength, namely $f_{c}$ and $f_{c t m o}$, can be noticed. This was expected since the displacement behavior for reinforced concrete girders are strongly dependent on the tension stiffening behavior and, consequently, on the tensile behavior of the concrete. Also, when viewing these results, it can be noticed that the random variable representing the yield strength was not updated in this process. Simulations were carried out considering this variable, however no noticeable changes were found. This was attributed to the fact that it is highly unlikely that the yield strength has any effect on the girder response for the measured load intensities. Table 2.2 summarizes the results of the simulations; it shows that, for most of the analyzed parameters, there was good repeatability in the updating procedure.

Some subtle changes were found in the updated distributions for the random variables which control the degradation mechanisms, $K$ and $\nu$. With regard to these results it should be noted that the use of information from only one point in the life of the structure, in this case $t=38$ years, cannot accurately characterize these variables. The reason behind 
this is that, simply put, the response of reinforced concrete structures are dependent on two main components: the concrete and the reinforcement. So the updating procedure is capable, at best, of determining the concrete and reinforcement properties for the concrete girder at the time of the test. If the degradation model consists on reinforcement corrosion, as is the case considered here, it is very difficult to infer if the reinforcement suffered corrosion or if its area is different from the specified nominal area. Furthermore, with a degradation model that considers a corrosion initiation time it is even more difficult to estimate the parameters based on a single point in time. For instance, given that one knows the initial reinforcement area and, through testing, determines the reinforcement area for a point in time, there still would be no way of knowing if this was the result of the initiation time being lower than expected or if the corrosion rate is higher than anticipated. Therefore, if the objective behind the testing is determining the occurrence and/or progress of the degradation mechanisms then, testing should be carried out at different ages of the structure.

In the present case study, in which test data is only available at one age of the structure, there is the possibility of improving these results by taking measurements of the carbonation front and using this information to update the random variable $K$. By having a better identified $K$ it is easier to obtain an estimate for the corrosion rate $\nu$ through the structural testing results. However, this still only applies if the corrosion has, in fact, initiated and the loss of rebar cross section is sufficient to change the girder stiffness. In the case where there is no evidence of reinforcement corrosion, the displacement data can still be successfully used to update the random variables that determine the time independent behavior of the girder. 

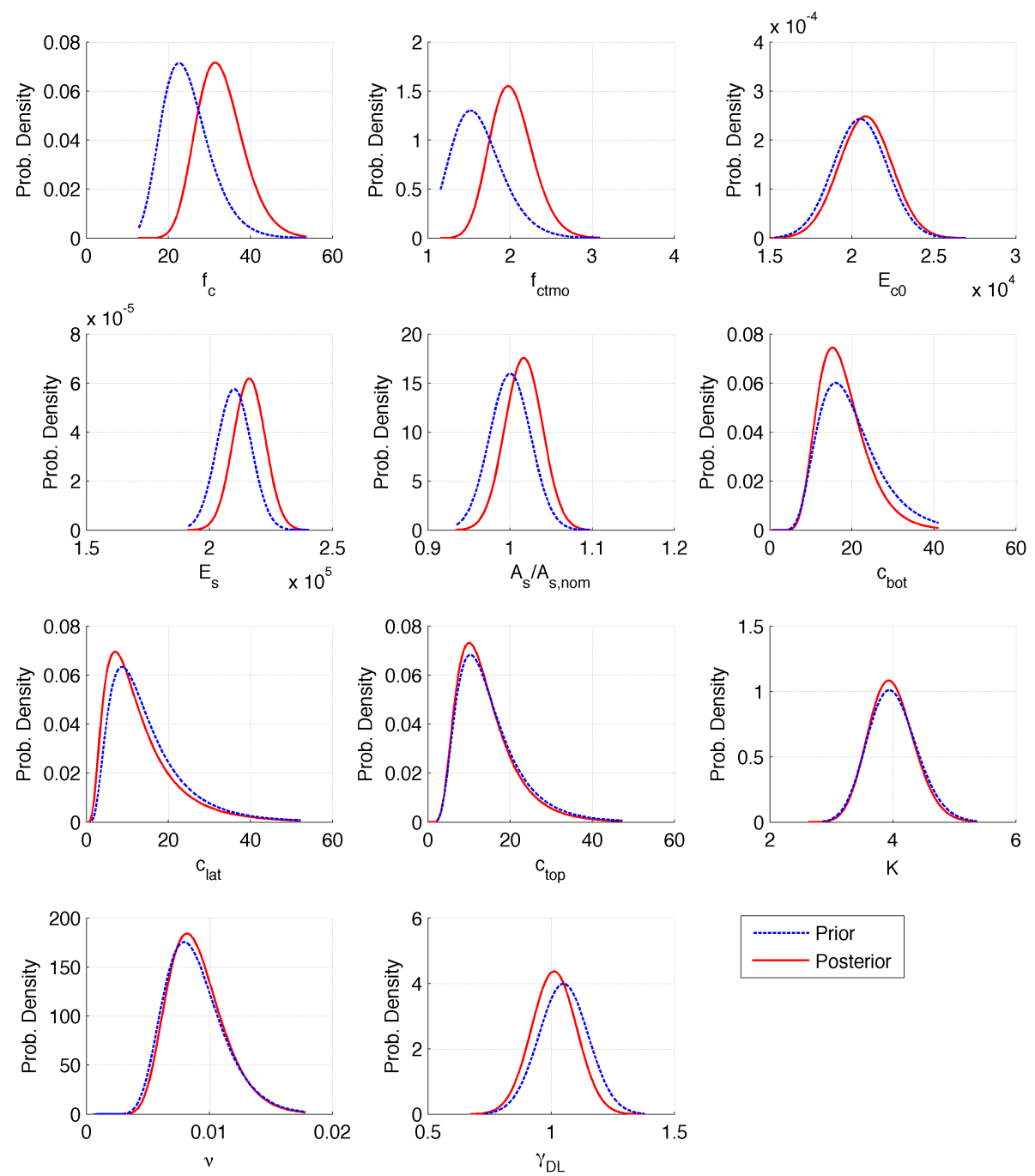

Figure 2.5.: Bayesian updating results based on the measured displacement data.

During the field testing of the studied railway bridge, carbonation front measurements were carried out through phenolphthalein tests. This data, along with the measured displacements, was used in the Bayesian updating procedure by applying the combined likelihood equation (eq. 2.29). For the likelihood of the carbonation depth information, due to the test uncertainty and modeling errors, a multiplicative error (eq. 2.28) with c.o.v. of $20 \%$ was considered. The phenolphthalein tests revealed carbonation depths ranging from 0 to $7.3 \mathrm{~mm}$ with a mean value of $3 \mathrm{~mm}$, which indicate that corrosion most likely had not initiated in the longitudinal reinforcement at that time. This hypothesis was corroborated by visual inspections that were also carried out during testing. Due to this and, also, for comparison reasons, another run of the updating algorithm was also carried out in which the degradation was not considered in the behavioral model. 
These two updating cases were, again, repeated ten times. Table 2.2 shows the mean and c.o.v's of the random variables' parameters (mean and standard deviation) for the three updating cases. The integration of the carbonation depth information results in significant changes in the distribution for the random variable $K$. However, during the different runs of the updating algorithm with this data, the corrosion rate and reinforcement cover distributions showed significant variability. This is most likely attributed to difficulties in sampling algorithm when dealing with variables with low influence on the response in low likelihood cases. Also, even though we have data that better identifies the $K$ random variable, the corrosion initiation can still occur due to lower cover values. Since only one point in time is being considered, there still remains uncertainty involving the response. As the main objective of the updating process is to obtain an improved strength prediction based on measured displacement, Monte Carlo simulations of the cross-sectional bending strength were carried out to determine the predicted strength at the time of the testing. For these simulations the mean values of the random variable parameters were used, additionally, a model uncertainty factor with normal distribution $(\mu=1.01, \sigma=0.046)$ was used [52]. Figure 2.6 shows the probability density of the strength prediction considering the random variables with the prior (initial) and the updated distributions for the three cases considered. These results demonstrate that, for the considered study case, the stiffness information yielded significant changes in the predicted cross sectional bending strength. The cases that consider the carbonation depth data and no degradation resulted in very similar distributions which is consistent, since the phenolphthalein tests indicated that no damage was expected at the time of testing. Also, the case that considers only the displacement information does not differ significantly from the other updated cases. 


\begin{tabular}{|c|c|c|c|c|c|c|c|}
\hline \multirow{2}{*}{\multicolumn{2}{|c|}{$\begin{array}{c}\text { Model } \\
\text { Parameters }\end{array}$}} & \multicolumn{2}{|c|}{ Displacement } & \multicolumn{2}{|c|}{ Displacement \& Phenolphthalein } & \multicolumn{2}{|c|}{ Displacement \& No Degradation } \\
\hline & & Mean & C.O.V. & Mean & C.O.V. & Mean & C.O.V. \\
\hline \multirow{2}{*}{$f_{c}(\mathrm{MPa})$} & $\overline{\mu \mu}$ & 34.12 & $5.7 \%$ & 32.76 & $9.5 \%$ & 32.85 & $3.6 \%$ \\
\hline & $\sigma$ & 6.07 & $16.8 \%$ & 4.46 & $19.5 \%$ & 5.79 & $8.4 \%$ \\
\hline \multirow{2}{*}{$f_{c t m o}(\mathrm{MPa})$} & $\mu$ & 2.00 & $4.5 \%$ & 1.97 & $10.3 \%$ & 2.02 & $4.5 \%$ \\
\hline & $\sigma$ & 0.27 & $16.6 \%$ & 0.20 & $34.5 \%$ & 0.26 & $8.3 \%$ \\
\hline \multirow{2}{*}{$E_{c 0}(\mathrm{MPa})$} & $\mu$ & $2.13 \times 10^{4}$ & $2.8 \%$ & $2.14 \times 10^{4}$ & $3.7 \%$ & $2.08 \times 10^{4}$ & $2.7 \%$ \\
\hline & $\sigma$ & $1.74 \times 10^{3}$ & $16.0 \%$ & $1.17 \times 10^{3}$ & $20.8 \%$ & $1.61 \times 10^{3}$ & $9.5 \%$ \\
\hline \multirow{2}{*}{$E_{s}(\mathrm{MPa})$} & $\mu$ & $2.17 \times 10^{5}$ & $1.2 \%$ & $2.17 \times 10^{5}$ & $2.1 \%$ & $2.16 \times 10^{5}$ & $0.7 \%$ \\
\hline & $\sigma$ & $6.02 \times 10^{3}$ & $10.7 \%$ & $5.19 \times 10^{3}$ & $14.5 \%$ & $6.45 \times 10^{3}$ & $13.7 \%$ \\
\hline \multirow{2}{*}{$A s / A s_{n o m}$} & $\mu$ & 1.02 & $0.9 \%$ & 1.03 & $1.1 \%$ & 1.02 & $0.4 \%$ \\
\hline & $\sigma$ & 0.024 & $7.3 \%$ & 0.018 & $24.7 \%$ & 0.023 & $8.4 \%$ \\
\hline \multirow{2}{*}{$c_{b o t}(\mathrm{~mm})$} & $\mu$ & 19.53 & $9.3 \%$ & 17.78 & $15.3 \%$ & 17.98 & $6.1 \%$ \\
\hline & $\sigma$ & 6.51 & $16.1 \%$ & 4.69 & $17.9 \%$ & 6.15 & $12.1 \%$ \\
\hline \multirow{2}{*}{$c_{t o p}(\mathrm{~mm})$} & $\mu$ & 14.92 & $19.5 \%$ & 14.39 & $27.0 \%$ & 13.44 & $17.3 \%$ \\
\hline & $\sigma$ & 8.80 & $19.9 \%$ & 6.25 & $30.1 \%$ & 9.96 & $15.3 \%$ \\
\hline \multirow{2}{*}{$c_{\text {lat }}(\mathrm{mm})$} & $\mu$ & 18.87 & $10.7 \%$ & 16.70 & $34.5 \%$ & 14.24 & $10.6 \%$ \\
\hline & $\sigma$ & 7.67 & $20.8 \%$ & 6.02 & $34.4 \%$ & 7.33 & $18.4 \%$ \\
\hline \multirow{2}{*}{$K\left(\mathrm{~mm} \cdot \mathrm{yr}^{-1 / 2}\right)$} & $\mu$ & 3.92 & $3.7 \%$ & 1.84 & $7.7 \%$ & - & - \\
\hline & $\sigma$ & 0.35 & $12.2 \%$ & 0.06 & $41.0 \%$ & - & - \\
\hline \multirow{2}{*}{$\nu\left(\mathrm{cm} \cdot \mathrm{yr}^{-1}\right)$} & $\mu$ & $8.82 \times 10^{-3}$ & $7.8 \%$ & $8.68 \times 10^{-3}$ & $25.7 \%$ & - & - \\
\hline & $\sigma$ & $2.32 \times 10^{-3}$ & $10.4 \%$ & $1.78 \times 10^{-3}$ & $35.7 \%$ & - & - \\
\hline \multirow{2}{*}{$\gamma_{D L}$} & $\mu$ & 1.01 & $2.9 \%$ & 1.02 & $6.8 \%$ & 1.01 & $3.5 \%$ \\
\hline & $\sigma$ & 0.09 & $7.3 \%$ & 0.07 & $18.4 \%$ & 0.09 & $6.5 \%$ \\
\hline
\end{tabular}

Table 2.2.: Mean and coefficient of variation of the distribution parameters for the random variables obtained in the ten runs of the updating algorithm for the three considered cases.

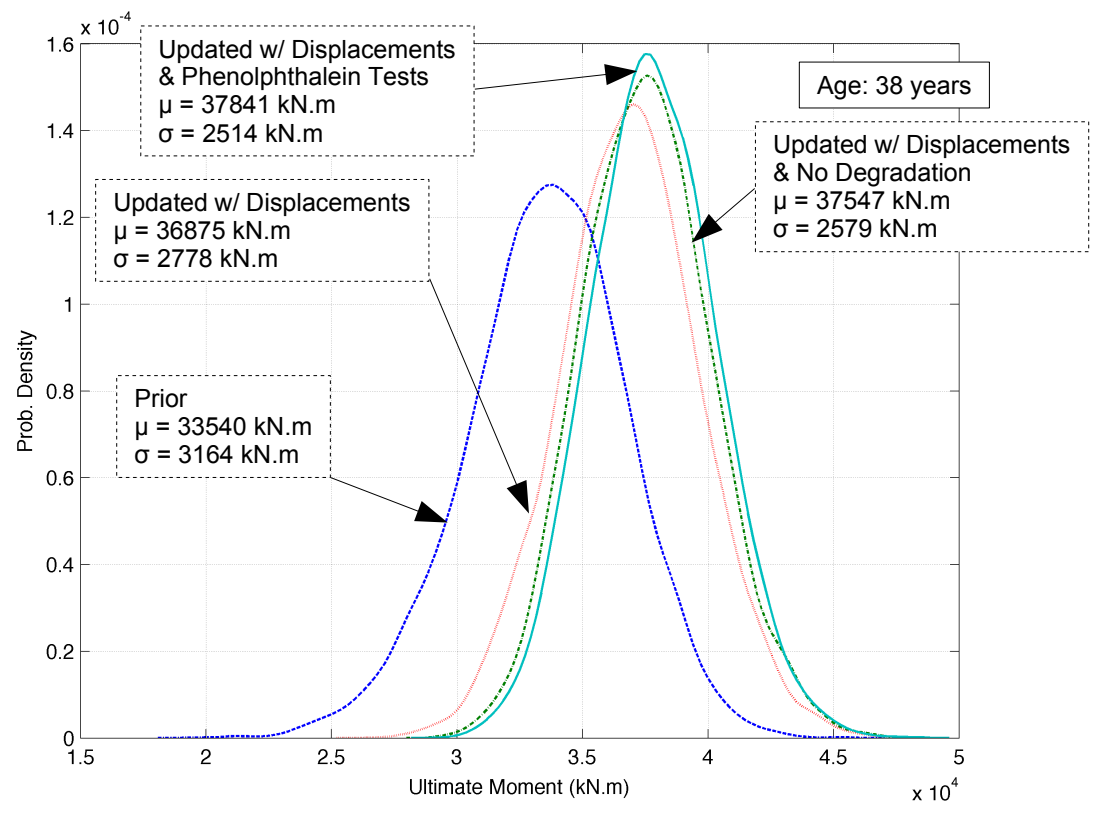

Figure 2.6.: Predicted ultimate bending moment of the cross section considering updating with different information. 


\subsubsection{Model Updating Using Data from Multiple Tests}

As commented earlier, the degradation mechanism based on reinforcement corrosion requires at least three points in time to be determined. With this objective, a set of testing scenarios were used to explore the potential of using testing data for change in structural response detection and updating of damage mechanism parameters. The testing scenarios, shown in Table 2.3, consider that testing is carried out $\Delta t$ years after the original test and that a change in response, when compared to the original test, was recorded.

In the first scenario, no change in response was detected in the future tests carried out in five year intervals. This scenario does not allow to make any inference regarding the corrosion rate, however it does give some information regarding the carbonation coefficient (corrosion did not initiate for at least 10 years after the original test). Scenario II has tests carried out every ten years with no change in the first test and a $10 \%$ increase in the last test. For scenario III, the tests are carried out every five years, with displacements, respectively, 5 and $10 \%$ higher than the original. Comparing these two scenarios, it may be inferred that the corrosion initiation times are different, with corrosion initiating first in scenario III, and the corrosion rates are similar (10\% change in response in a 10 year period). Finally, for scenario IV, testing is done ten year intervals with changes of 5 and $10 \%$ which implies a lower corrosion rate than scenarios II and III and an initiation time close to scenario III.

\begin{tabular}{|c|c|c|c|}
\hline$\Delta t$ (years) & 5 & 10 & 20 \\
\hline Scenario & \multicolumn{3}{|c|}{ Change in Response } \\
\hline I & $0 \%$ & $0 \%$ & \\
\hline II & & $0 \%$ & $+10 \%$ \\
\hline III & $+5 \%$ & $+10 \%$ & \\
\hline IV & & $+5 \%$ & $+10 \%$ \\
\hline
\end{tabular}

Table 2.3.: Scenarios considered for updating using three points in time.

The updating routine for each scenario used the combined likelihood of the data obtained in the three tests, resulting in a set of 30 observations. For simplicity and, also, taking into consideration the results obtained in the previous section, the uncertainty of the concrete cover was not considered. Therefore the nominal values were used in this analysis, which allowed for a better characterization of the $K$ (carbonation coefficient) parameter. Similarly as in the previous updating procedure, for each scenario, ten consecutive runs of the algorithm were carried out.

Table 2.4 shows the mean and c.o.v.'s of the distribution parameters for the updated random variables. From these results it can be seen that the model parameters, $f_{c}$, $f_{c t m o}, E_{c 0}, E_{s}, A_{S} / A_{s_{n o m}}$, and $\gamma_{D L}$, do not show significant changes in the four considered scenarios. For the carbonation constant, $K$, the results show, the lower values associated with scenarios I and II and slightly higher values associated with scenarios III and IV. 
Considering a reinforcement cover of $20 \mathrm{~mm}$ the initiation times for the four scenarios would, respectively, be $30.2,28.5,26.4$, and 27.8 years. This shows that for this cover depth, changes in initiation time vary from 2 to 4 years, since the cross section has reinforcement layers with larger covers, it is reasonable to assume that changes of 5 years in the initiation time would not generate significant changes in the $K$ values.

With regard to the corrosion rate, $\nu$, scenarios II and III presented similar values, scenario IV showed lower values than the aforementioned scenarios. This is consistent with the information from the scenarios, since scenario IV showed only $5 \%$ increase in displacement in a 10 year period as opposed to a $10 \%$ increase in the two other scenarios for the same period. As already mentioned, scenario I does not allow to make any inferences on the corrosion rate.

To better evaluate the effects of this variable and of the updating process in the lifetime flexural behavior of the girder, a Monte Carlo simulation was run using the mean values for the distribution parameters shown in Table 2.4. For comparison, a simulation using the prior distribution information was also carried out.

Figure 2.7 shows the evolution, in terms of mean values of the ultimate bending moment, $M_{u}$, during a timespan of 100 years. A relative damage was calculated by dividing the obtained mean values of $M_{u}$ at the different times by the initial value ( $t=0$ years), which allows for further illustration of the differences in the simulated damage rates. The mean values for the different updating scenarios at the ages before damage initiation demonstrate, again, that there is a reasonable consistency in the parameters, which control the time independent behavior of the model. After the damage initiation time, there is a stage in which the damage rate is variable, which is due to the depassivation progression through the different reinforcement layers. After this stage, it can be seen that the rates are fairly constant. It can be seen that at this stage, for instance, scenarios II and III degrade at a faster rate than the remaining scenarios, which is consistent with the statements above. 


\begin{tabular}{|c|c|c|c|c|c|c|c|c|c|}
\hline \multirow{2}{*}{\multicolumn{2}{|c|}{$\begin{array}{c}\text { Model } \\
\text { Parameters }\end{array}$}} & \multicolumn{2}{|c|}{ Scenario I } & \multicolumn{2}{|c|}{ Scenario II } & \multicolumn{2}{|c|}{ Scenario III } & \multicolumn{2}{|c|}{ Scenario IV } \\
\hline & & Mean & C.O.V. & Mean & C.O.V. & Mean & C.O.V. & Mean & C.O.V. \\
\hline \multirow{2}{*}{$f_{c}(\mathrm{MPa})$} & $\mu$ & 36.32 & $8.3 \%$ & 34.43 & $7.5 \%$ & 35.74 & $5.6 \%$ & ב34.31 & $11.6 \%$ \\
\hline & $\sigma$ & 5.34 & $19.0 \%$ & 5.13 & $19.7 \%$ & 5.37 & $14.1 \%$ & 5.16 & $13.0 \%$ \\
\hline \multirow{2}{*}{$f_{c t m o}(\mathrm{MPa})$} & $\mu$ & 1.80 & $8.1 \%$ & 1.81 & $5.6 \%$ & 1.75 & $5.0 \%$ & 1.84 & $7.9 \%$ \\
\hline & $\sigma$ & 0.23 & $20.3 \%$ & 0.25 & $20.3 \%$ & 0.22 & $10.9 \%$ & 0.22 & $15.5 \%$ \\
\hline \multirow{2}{*}{$E_{c 0}(\mathrm{MPa})$} & $\mu$ & $2.18 \times 10^{4}$ & $5.2 \%$ & $2.20 \times 10^{4}$ & $2.9 \%$ & $2.18 \times 10^{4}$ & $3.0 \%$ & $2.11 \times 10^{4}$ & $2.0 \%$ \\
\hline & $\sigma$ & $1.44 \times 10^{3}$ & $15.7 \%$ & $1.36 \times 10^{3}$ & $21.4 \%$ & $1.49 \times 10^{3}$ & $17.4 \%$ & $1.45 \times 10^{3}$ & $16.9 \%$ \\
\hline \multirow{2}{*}{$E_{s}(\mathrm{MPa})$} & $\mu$ & $2.25 \times 10^{5}$ & $1.3 \%$ & $2.25 \times 10^{5}$ & $0.6 \%$ & $2.23 \times 10^{5}$ & $1.0 \%$ & $2.25 \times 10^{5}$ & $1.4 \%$ \\
\hline & $\sigma$ & $5.36 \times 10^{3}$ & $11.7 \%$ & $5.65 \times 10^{3}$ & $23.9 \%$ & $5.58 \times 10^{3}$ & $22.1 \%$ & $5.51 \times 10^{3}$ & $15.9 \%$ \\
\hline \multirow{2}{*}{$A s / A s_{n o m}$} & $\mu$ & 1.04 & $1.3 \%$ & 1.04 & $0.9 \%$ & 1.03 & $0.9 \%$ & 1.03 & $2.0 \%$ \\
\hline & $\sigma$ & 0.021 & $20.9 \%$ & 0.021 & $16.9 \%$ & 0.020 & $25.5 \%$ & 0.021 & $15.1 \%$ \\
\hline \multirow{2}{*}{$K\left(\mathrm{~mm} \cdot \mathrm{yr}^{-1 / 2}\right)$} & $\mu$ & 3.64 & $1.8 \%$ & 3.75 & $2.8 \%$ & 3.89 & $2.3 \%$ & 3.79 & $2.7 \%$ \\
\hline & $\sigma$ & 0.21 & $10.6 \%$ & 0.20 & $21.2 \%$ & 0.25 & $20.0 \%$ & 0.26 & $19.2 \%$ \\
\hline \multirow{2}{*}{$\nu\left(\mathrm{cm} \cdot \mathrm{yr}^{-1}\right)$} & $\mu$ & $8.38 \times 10^{-3}$ & $15.2 \%$ & $8.88 \times 10^{-3}$ & $10.1 \%$ & $8.76 \times 10^{-3}$ & $6.4 \%$ & $7.84 \times 10^{-3}$ & $15.9 \%$ \\
\hline & $\sigma$ & $2.43 \times 10^{-3}$ & $43.3 \%$ & $1.86 \times 10^{-3}$ & $14.9 \%$ & $2.07 \times 10^{-3}$ & $22.7 \%$ & $1.91 \times 10^{-3}$ & $20.2 \%$ \\
\hline \multirow[b]{2}{*}{$\gamma_{D L}$} & $\mu$ & 1.05 & $1.8 \%$ & 1.03 & $5.1 \%$ & 1.04 & $2.0 \%$ & 1.07 & $4.7 \%$ \\
\hline & $\sigma$ & 0.08 & $13.0 \%$ & 0.08 & $27.8 \%$ & 0.08 & $9.9 \%$ & 0.09 & $17.1 \%$ \\
\hline
\end{tabular}

Table 2.4.: Mean values and coefficient of variation of the random variable parameters obtained in the ten runs of the updating procedure for the four considered scenarios.
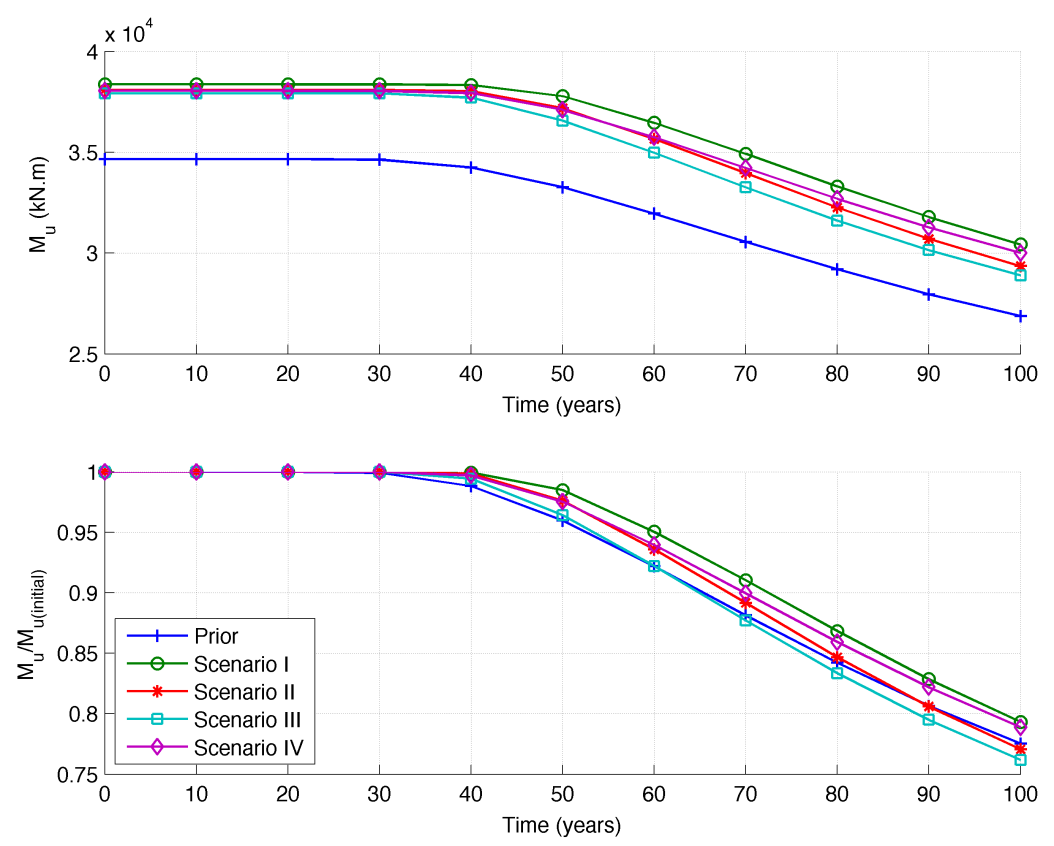

Figure 2.7.: Curves showing: the mean values of the ultimate bending moment $\left(M_{u}\right)$ and the relative damage $\left(M_{u} / M_{u(\text { initial })}\right)$ at different ages. 


\subsection{Final Considerations}

An approach to utilizing Bayesian updating techniques associated with a mechanical model for reinforced concrete cross sections to obtain improved strength predictions was shown. Using a layer-by-layer discretization of the reinforced concrete cross section, a behavioral model was established. Damage due to reinforcement corrosion was also modeled with the assumption that changes in behavior were solely based on the reduction of reinforcement area due to uniform corrosion. Bayesian updating using an approach proposed by Straub [90], which allows for the use of structural reliability methods for solving these types of problems, was used to update the random variables representing the behavioral model parameters using displacement information. Data obtained from field testing of a reinforced concrete railway bridge was used to test and present the methodology.

The measured response obtained in the tests showed a significantly stiffer response than predicted by the analysis model. Therefore, considerable changes were found in the prior and updated distributions of the model parameters. It should be noted that, the prior information was determined based on the available design documentation which contain many uncertainties, specially regarding concrete strength. As shown in Figure 2.5, the parameters regarding the concrete compressive and tensile strength, $f_{c}$ and $f_{\text {ctmo }}$, suffered the biggest changes due to the new information.

Simulation results show that the strength prediction also showed significant changes due to the stiffness information. Two other simulations were run, one considering the measured carbonation depths and, another, not considering the degradation mechanisms. The differences between the strength predictions on these three updated cases, as shown in Figure 2.6 are not very significant. This is due to the fact that at the age the structure was tested, 38 years, no significant damage was expected by any of the models. Therefore the use of only displacement data in the simulations should be used with care.

Model updating considering three points at different ages of the structure showed that one is able to update the prediction model solely on the displacement data. However, the use of other sources of information, such as the carbonation depths (for this specific case), can considerable improve the prediction accuracy. For example, according to the carbonation depths that were obtained, corrosion initiation was only expected at around the age of 110 years. Scenario I demonstrates that, if the model expects a certain damage at the time of the test, and that damage is not detected, it assumes that the damage is imminent. This is, logically, because the displacement data can only give information regarding the occurrence of damage. It cannot give information regarding the corrosion initiation time unless damage has already occurred. Therefore, this methodology would be better used if associated with other NDT that are able to better determine specific degradation model parameters [55, 39]. 
Degradation was modeled considering only reinforcement corrosion due to depassivation of the reinforcement by carbonation. However the methodology may be applied to other types of degradation mechanisms. Also, the effects of reinforcement corrosion took into consideration only the loss of rebar area. There are other models available that take into consideration the loss of area and the changes in the bond strength of the reinforcement [22]. The application of these models and the inclusion of other types of reinforcement corrosion are suggested for future investigations.

This work assumes an error function with a bias factor of 0.85 and a $10 \%$ coefficient of variation. This relatively large coefficient of variation was in part due to uncertainties in the axle loading measurements. However, it should be noted that such a large error can cause problems when trying to detect subtler changes in the structural behavior. It is suggested that further studies regarding the effects of different measurement errors should be carried out. As previously mentioned, the bias factor was adopted as a measure of safety as to not overestimate strength due to a stiffness that is not due to the considered material properties. For instance, issues in the bridge bearings [16] and bridge-track interaction in railways [35] have shown to alter the apparent structural stiffness. It should be noted that this is a very important aspect that must be taken into consideration when updating strength prediction based on stiffness measurements.

The Bayesian updating methodology based on subset simulation showed satisfactory results. It significantly decreases the number of model evaluations necessary for the solution of the updating problem, specially when compared to Markov Chain Monte Carlo methods such as the Metropolis algorithm [59]. Some issues were found when dealing with larger datasets, however the algorithm was adequate for the suggested methodology. The possibility of obtaining improved performance in this method when dealing with larger datasets as investigated by Betz et al. [12] is a suggestion for future works in this area. This methodology can be specially useful if other sources of information are to be considered. 


\section{Structural Health Monitoring of the Jaguari River Bridge}

A Structural Health Monitoring system was developed as part of the research carried out during this doctorate period. The monitoring system of the Jaguari River Bridge was idealized as a prototype for different types of structural monitoring applications. This chapter describes the procedures that were carried out as part of the development of this system and the analysis and interpretation of some of the obtained results.

\subsection{Description of the Structure}

The Jaguari River Bridge was chosen to host a long-term monitoring system due to it's location attending the project demands for safety against burglary and vandalism of the system. Another reason for choosing this bridge was its structural type, which was representative of many other Brazilian highway bridges.

\subsubsection{Location and Bridge Layout}

The bridge chosen to house the structural monitoring system was the southbound Jaguari River Bridge in the Fernão Dias Highway. This highway, which has a significant traffic demand, links the cities of São Paulo and Belo Horizonte which are cities of great economic importance. The structure is located close to the city of Extrema in the State of Minas Gerais located 10 km São Paulo-Minas Gerais border (Figure 3.1).

As shown in Figures 3.2 and 3.3, the bridge crosses the Jaguari River ravine in a downhill and curved section of the highway. The radius of the curve is of $305.35 \mathrm{~m}$ at the centerline and the hill has a grade of $5.9 \%$. Also, due to the horizontal curve, there is a superelevation with a maximum of $8 \%$ grade as illustrated in Figure 3.4.

\subsubsection{Superstructure}

The bridge's superstructure, as shown in Figure 3.4, is composed mainly of the two longitudinal girders and a deck slab. The two longitudinal reinforced concrete girders are 


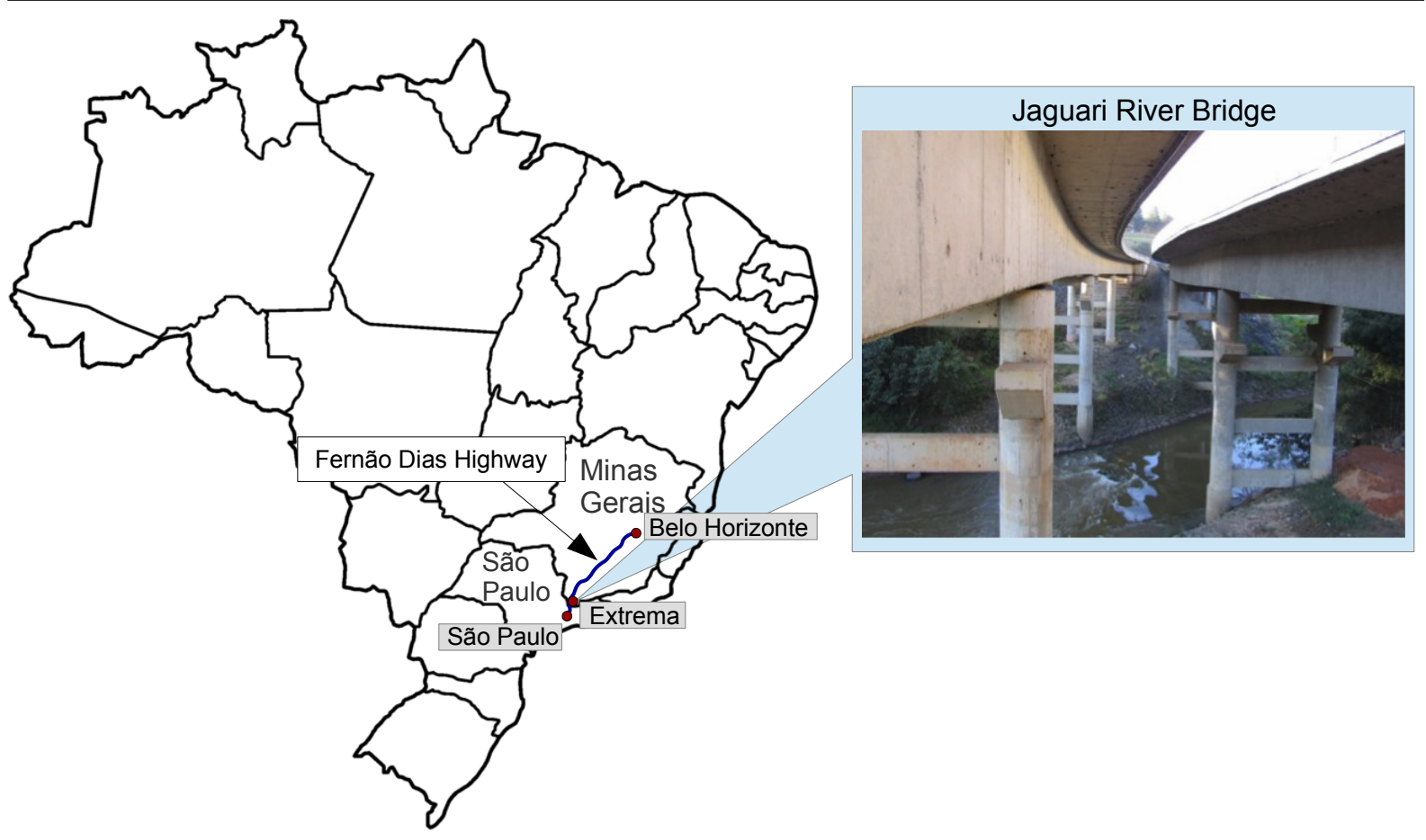

Figure 3.1.: Location of the Jaguari River Bridge.

continuous over the five spans of the $122 \mathrm{~m}$ crossing. These girders are $2.80 \mathrm{~m}$ in height and have variable widths that range from $0.4 \mathrm{~m}$ at the midspan to $1.0 \mathrm{~m}$ at the supports. The $11.70 \mathrm{~m}$ wide deck slab contains one shoulder and two traffic lanes. The deck has a thickness that varies from $0.24 \mathrm{~m}$ to $0.40 \mathrm{~m}$ with an asphalt pavement approximately $0.06 \mathrm{~m}$ thick and reinforced concrete New Jersey barriers on the sides. Transverse beams, connecting the two main girders, are distributed throughout the bridge with spacing ranging form 6 to $6.7 \mathrm{~m}$ depending on the span. The transverse beam dimensions and positioning are shown in Figures 3.4 and 3.3, respectively. At the ends of the structure there are lateral wing walls dug into the abutments. A reinforcement cover of $2.5 \mathrm{~cm}$ was specified in the design drawings for these superstructure elements.

Regarding the concrete specifications, the design documentation specified a characteristic compressive strength, $f_{c k}$, of $25 \mathrm{MPa}$. During construction, for each stage of concrete pouring, a batch of fresh concrete samples was taken for posterior curing and strength testing. This information, which was taken from the as-built documentation of the structure, is summarized in Table 3.1. The testing was carried out according to the Brazilian Standard NBR-12655[2] and shows that the concrete used in the superstructure meets the design requirements. 


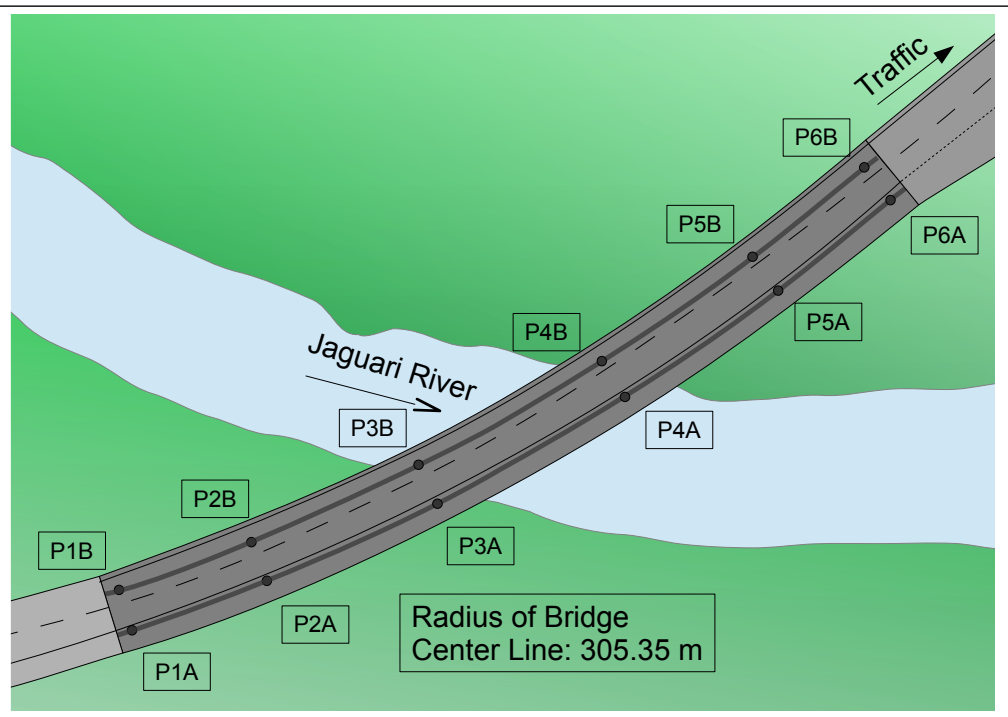

Figure 3.2.: Plan view of the Jaguari River Bridge.

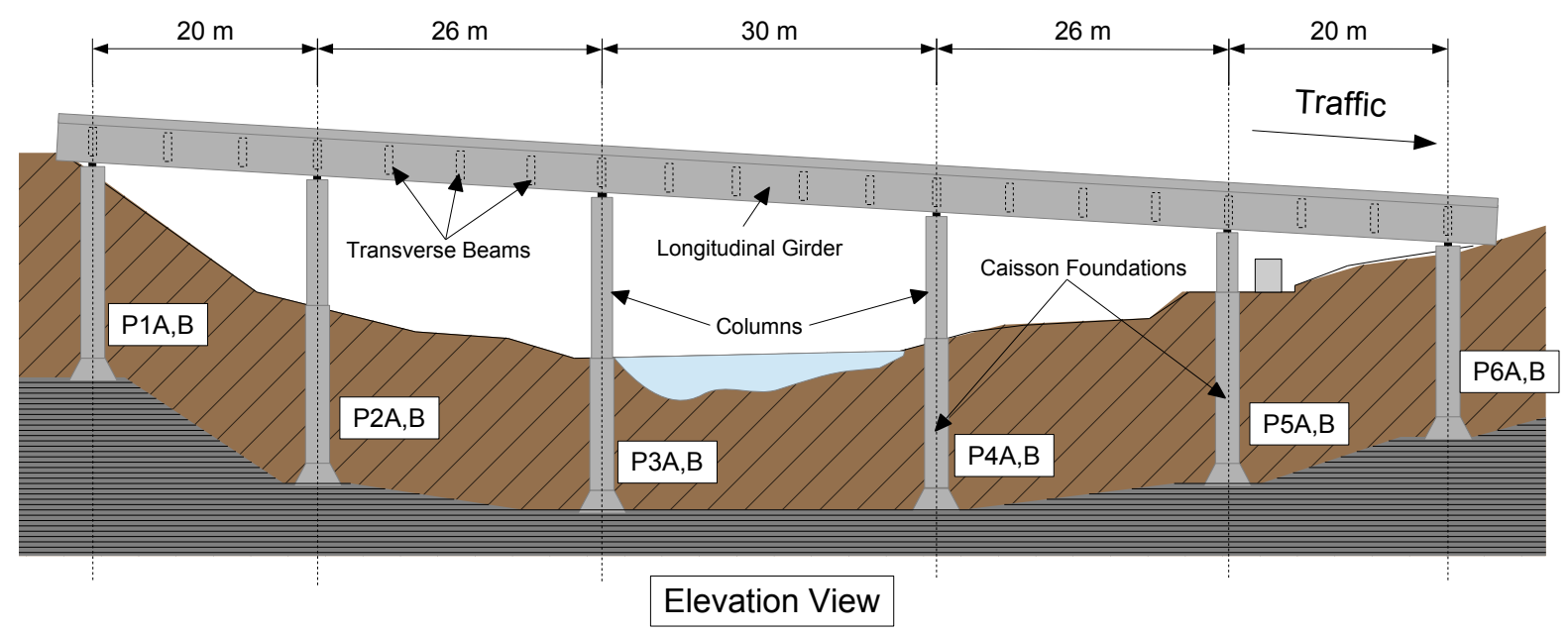

Figure 3.3.: Elevation view of the Jaguari River Bridge.

\subsubsection{Substructure}

Six pairs of columns with $1.20 \mathrm{~m}$ in diameter support the superstructure (Figures 3.2 and 3.3). Laminated elastomeric bearing pads are located at the top of each column. Concrete with a characteristic strength of $20 \mathrm{MPa}$ and reinforcement cover of $3.0 \mathrm{~cm}$ were specified in the design documents.

\subsubsection{Foundations and Abutments}

The six pairs of columns are supported by Caisson foundations which were dug until reaching the bedrock formation (Figure 3.3). The Caisson foundations have typically shafts with $1.40 \mathrm{~m}$ in diameter with bases of $2.00 \mathrm{~m}$ to $3.00 \mathrm{~m}$.

The bridge abutments were built by cutting and filling and the slopes are covered by 


\begin{tabular}{|c|c|c|}
\hline Batch & $f_{c(\text { mean })}(28$ days $)$ & $f_{\text {ck }(\text { estimated })}$ \\
\hline \hline 1 & $31.9 \mathrm{MPa}$ & $30 \mathrm{MPa}$ \\
\hline 2 & $38.5 \mathrm{MPa}$ & $35.54 \mathrm{MPa}$ \\
\hline 3 & $32.5 \mathrm{MPa}$ & $30.43 \mathrm{MPa}$ \\
\hline 4 & $30.6 \mathrm{MPa}$ & $28.02 \mathrm{MPa}$ \\
\hline 5 & $31.7 \mathrm{MPa}$ & $26.53 \mathrm{MPa}$ \\
\hline 6 & $30.2 \mathrm{MPa}$ & $30.28 \mathrm{MPa}$ \\
\hline 7 & $29.6 \mathrm{MPa}$ & $29.97 \mathrm{MPa}$ \\
\hline
\end{tabular}

Table 3.1.: Concrete strength test results for fresh concrete samples collected

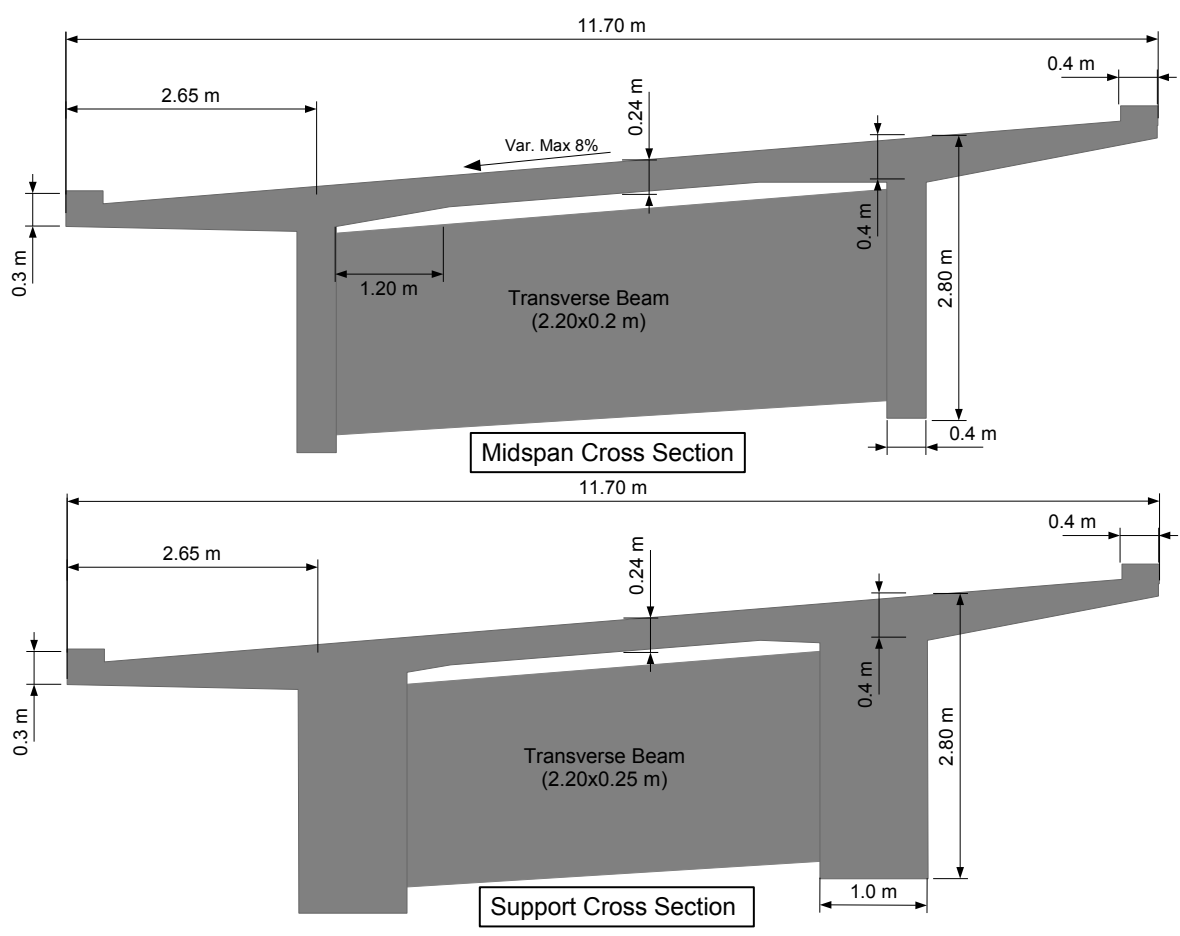

Figure 3.4.: Typical cross-sections of the Jaguari River Bridge.

a concrete covered enrockment layer. Transition slabs are present at each end of the bridge, and expansion joints are present in the connection between these slabs and the superstructure.

\subsubsection{Load Rating}

This structure was designed to withstand live loads of Class 45 from the Brazilian standard NBR 7188. Figure 3.5 illustrates the loading pattern, for this loading class, which consists of a three axle $450 \mathrm{kN}$ truck with a crowd loading of $5 \mathrm{kN} / \mathrm{m}^{2}$. 


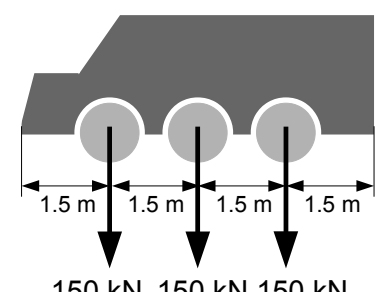

$150 \mathrm{kN} 150 \mathrm{kN} 150 \mathrm{kN}$

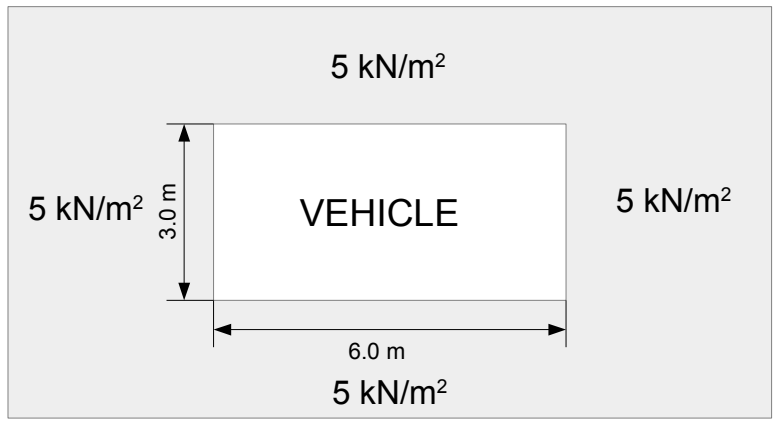

Figure 3.5.: Class 45 live loading pattern for the Brazilian standard NBR 7188.

\subsection{Visual Inspection, Testing, and Short-Term Monitoring}

As part of the development of the monitoring system an in-depth study of the structure was carried out. This study included visual inspections, semi and non-destructive testing, and short-term monitoring. In the following sections, key aspects of these procedures that are relevant to this work are detailed.

\subsubsection{Visual Inspections}

In order to make a first evaluation of the structure, visual inspection was carried out in 2010. During this procedure the elements were inspected for any signs of degradation and mapping of the crack visible patterns was developed. The results of these visual inspections are summarized in the sections that follow.

\subsubsection{Longitudinal Girders}

Inspection was carried out on the inner and outer surfaces of the longitudinal girders between columns P5 and P6. For the remaining spans, access was possible only for the outer surfaces. Isolated shrinkage cracks (Figure 3.6) were detected in several points throughout the girders. Flexural cracks were also detected at the midspans, as shown in Figure 3.7. Also, in some locations, stains from the runoff of infiltrations in the deck slab were detected. 


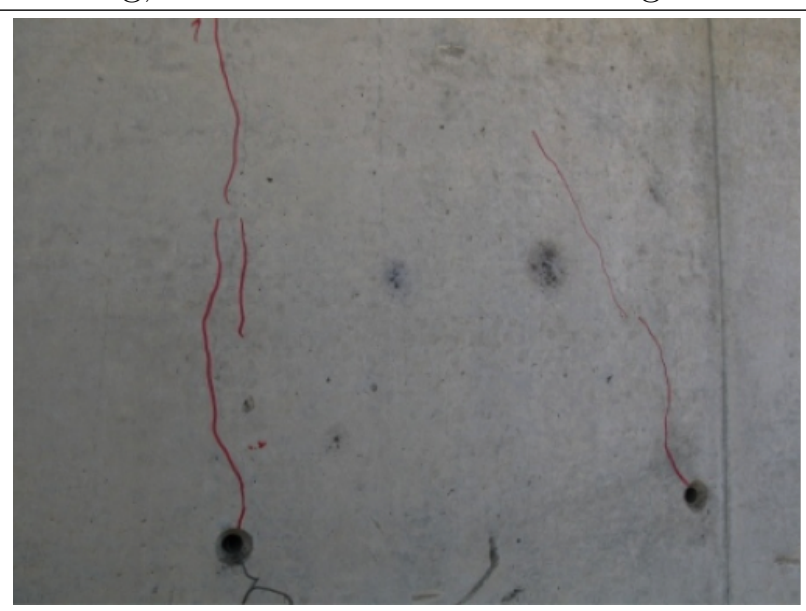

Figure 3.6.: Shrinkage cracks detected in the longitudinal girders.

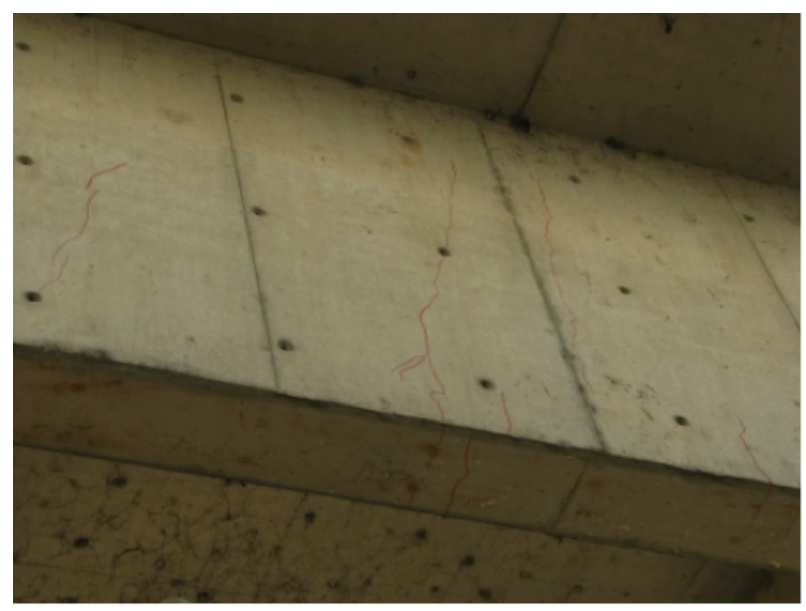

Figure 3.7.: Flexural cracks found in the longitudinal girders.

\subsubsection{Transverse Beams}

The main issues detected in the transverse beams were some isolated shrinkage cracks.

\subsubsection{Columns}

No signs of damage were detected on all six pairs of columns.

\subsubsection{Deck Slab and Pavement}

In the slab segment between columns P5 and P6 no damage was detected as shown in Figure 3.8. The slab section between columns P5 and P4 showed some shrinkage cracks. Also, a fairly large crack with infiltration was detected over the transverse beam closest to P4 in this section (Figure 3.9). A similar occurrence was detected over the transverse beam closest to P4 in the section between P3 and P4 (Figure 3.10). By inspecting the pavement it was found that these cracks went all the way up to the road surface. 


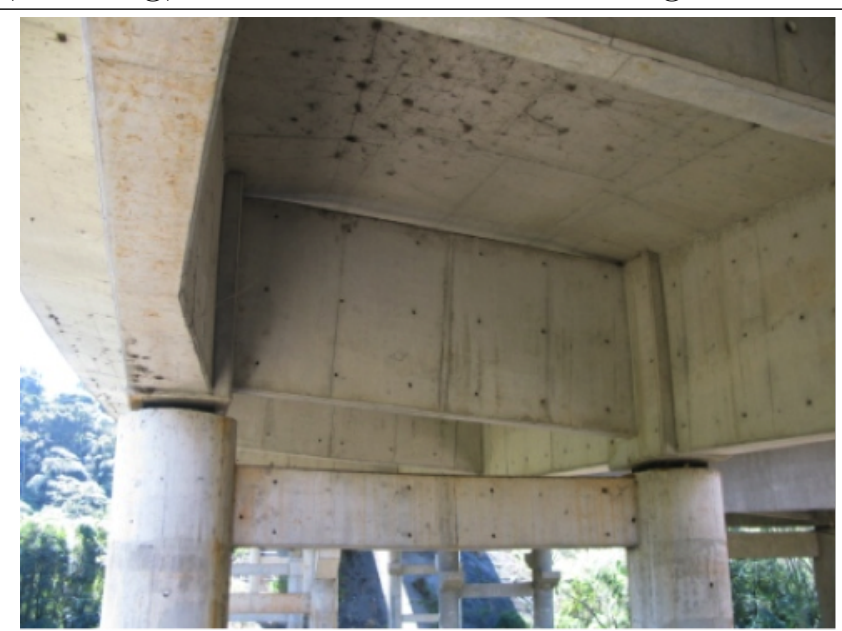

Figure 3.8.: View beneath the deck slab between columns P5 and P6.

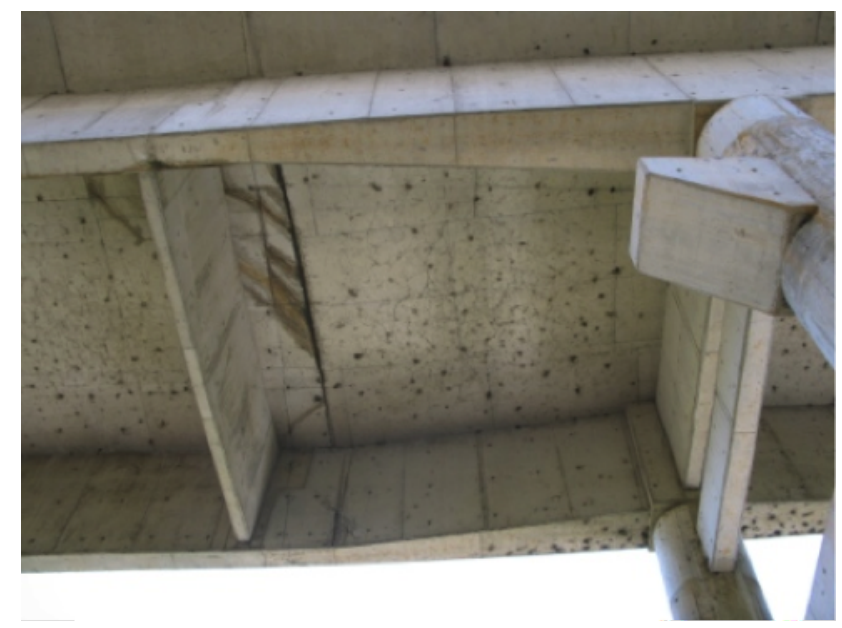

Figure 3.9.: Crack with infiltration close to column P4 (São Paulo side).

\subsubsection{Elastomeric Bearings}

Inspection of the laminated elastomeric bearings showed that these elements were in relatively good state. No signs of corrosion of the steel plates nor signs of crushing of the pads were found. However, some cracking was detected in a few of these elements (Figure 3.11(a)) and some acceptable distortion was also detected (Figure 3.11(b)).

\subsubsection{Semi and Non-Destructive Testing}

Semi-destructive and non-destructive testing were also carried out for the structure. Six core samples were extracted and taken to the laboratory for testing. The tests revealed a mean strength of $30.69 \mathrm{MPa}$ in the transverse beams and 26.51 $\mathrm{MPa}$ in the longitudinal girder. Phenolphthalein tests to determine the carbonation depth were also done. These tests showed a mean penetration of $17.12 \mathrm{~mm}$ with standard deviation of $6.19 \mathrm{~mm}$. 


\subsection{Visual Inspection, Testing, and Short-Term Monitoring}

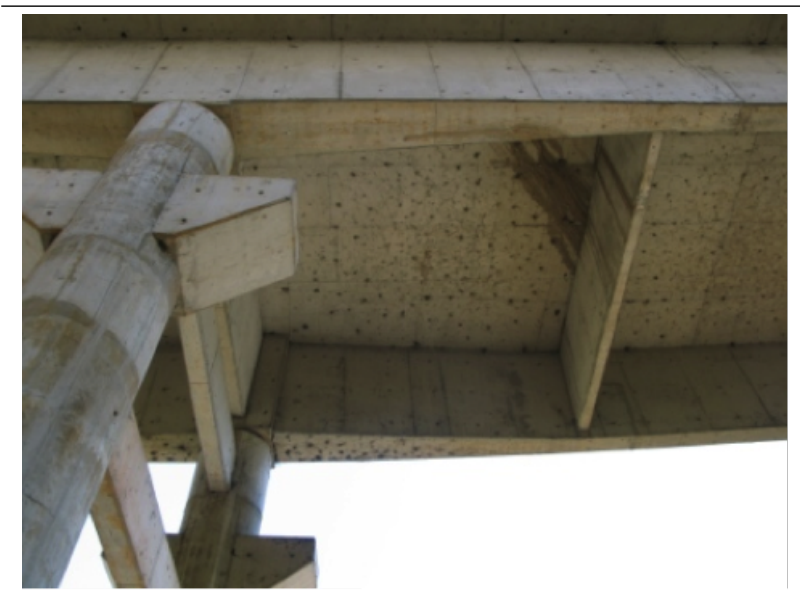

(a)

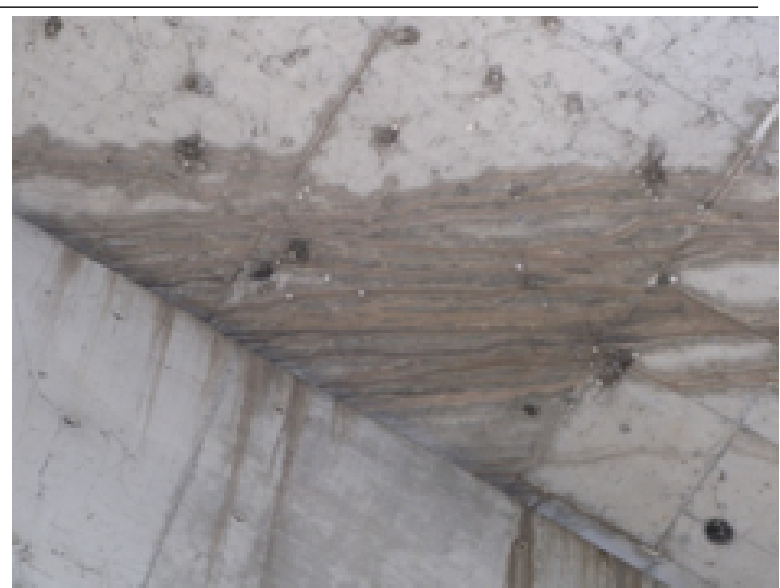

(b)

Figure 3.10.: Crack with infiltration close to column P4 (Belo Horizonte side): (a) overview, (b) zoomed in.

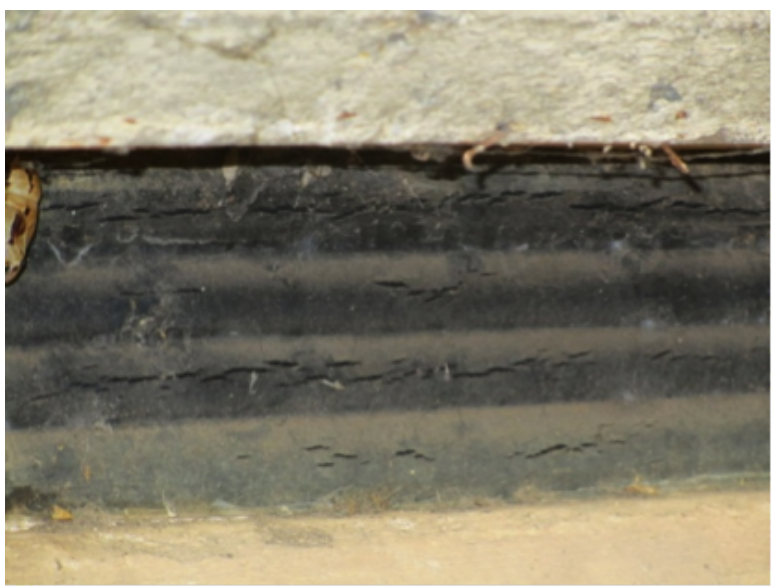

(a)

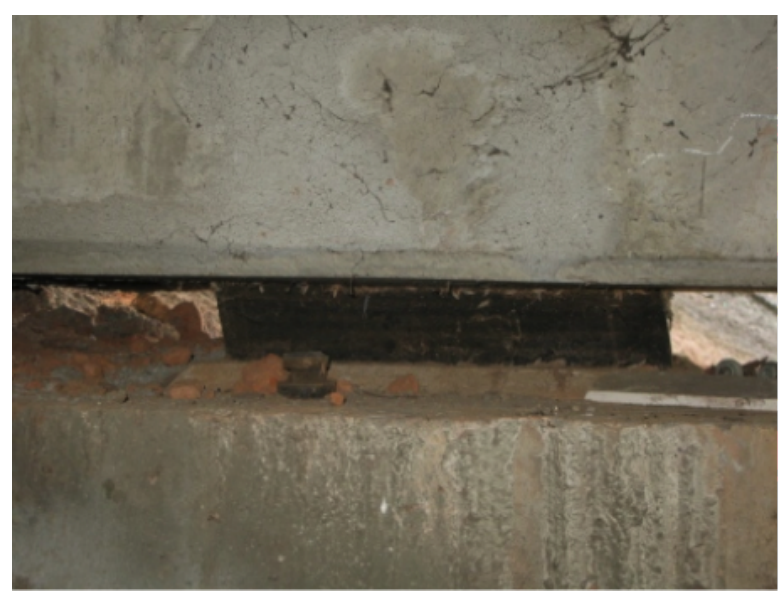

(b)

Figure 3.11.: Visual inspection of the laminated elastomeric bearing pads showing cracking (a) and distortion (b).

\subsubsection{Bridge Field Testing and Short-Term Monitoring}

Structural testing and short-term monitoring was carried out in this bridge in order to make an improved structural assessment and to make initial inquires regarding the positioning of the sensors for the long-term monitoring system. During this stage the bridge was instrumented and testing with a controlled load vehicle was executed. The bridge response under free traffic condition was also recorded at this time.

\subsubsection{Instrumentation}

Strain gauges, displacement sensors and accelerometers were used in the instrumentation of the short-term monitoring. Due to difficulty of access as well as limitations of the measurement equipment, only three spans of the bridge, the ones closest to the São Paulo 
end, were instrumented. The main objective of this instrumentation was to capture the global behavior of this structural system. Additional displacement sensors were applied in order to record the behavior of some of the cracks that were detected during the visual inspections.

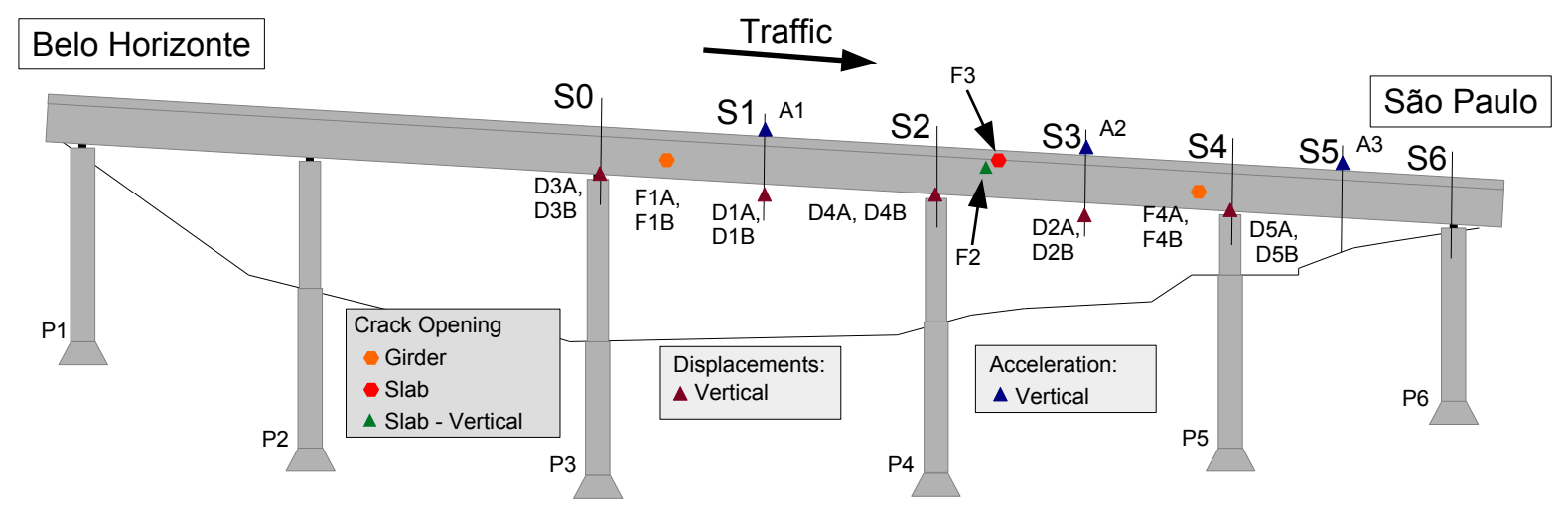

Figure 3.12.: Longitudinal view of the short-term instrumentation plan including the displacement sensor and accelerometers.

Figure 3.12 shows a longitudinal view of the instrumentation plan which includes the displacement sensors and accelerometers. Inductive displacement sensors were installed to measure the vertical displacements at selected midspans (Figure 3.13(a)) and supports (Figure 3.13(b)). Crack openings in some cracks found in the girders close to the supports were also monitored using displacement sensors (Figure 3.13(c)). Due to the configuration of the cracks, detected in the slabs, both crack opening and relative vertical displacement were measured, as presented in Figure 3.13(d).

As these sensor are relative displacement sensors they need to have a fixed reference point in order to operate. To measure the displacements at the midspans, a technique illustrated in Figure 3.14 was used. A spring is fixed to the structure and linked to a steel wire fixed to the ground. In this way, as the structure moves the spring expands or contracts keeping the steel wire in a fixed position. The displacement transducer is then fixed to the structure, with its movable rod in contact with a small plate fixed to the steel wire.

Piezoelectric accelerometers (Figure 3.15) were fixed to the girders to measure the vertical accelerations at three sections shown in Figure 3.12.

Strains were recorded at five cross-sections of the structure, two over the supports (S2 and S4) and three at midspans (S1, S3, and S5). The locations of these sections are illustrated in Figure 3.12. For each of the selected cross-sections, electrical strain gauges were installed, in the longitudinal direction, in three points along the height of the girders. The position of these gauges is specified in Figure 3.16.

To analyze the behavior of the deck slab, strain gauges were also installed in the longitudi- 


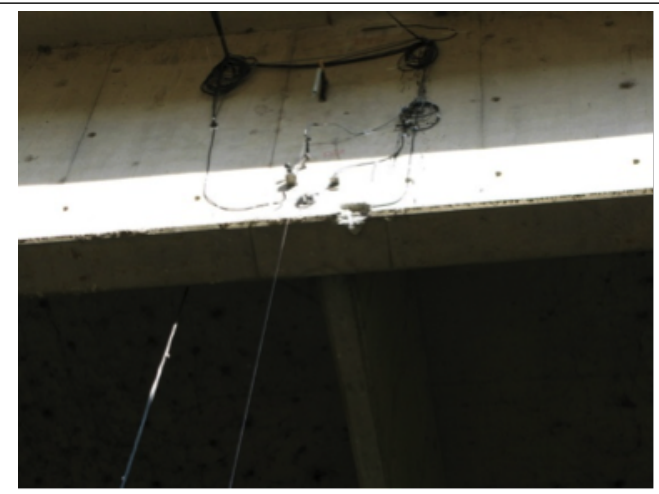

(a)

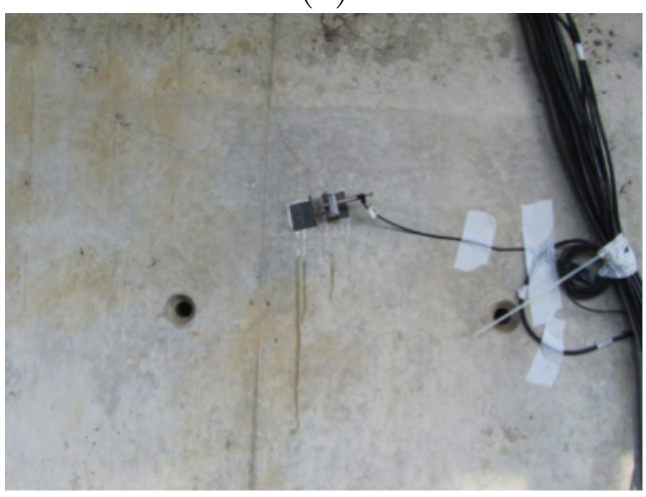

(c)

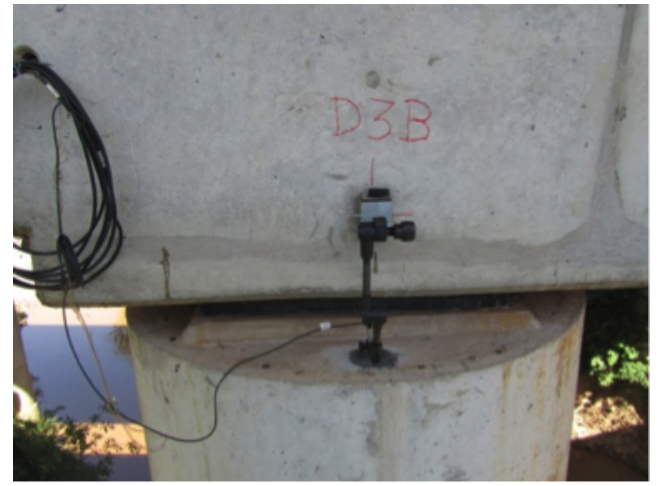

(b)

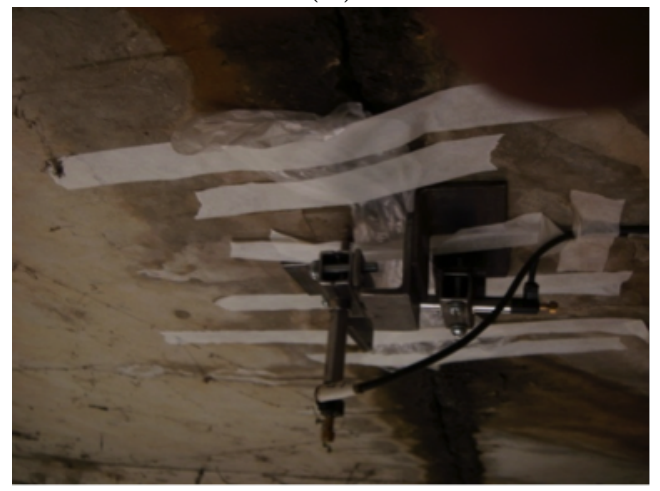

(d)

Figure 3.13.: Displacement sensors installed at the midspans (a), supports (b), cracks in the girders (c), and crack in the slab (d).

nal and transverse directions at the midsection of the slab in Section S3, between columns $\mathrm{P} 4$ and P5. Strain gauges were installed in the transverse and longitudinal direction in the top and bottom of midsection of the slab (3.16). Strain gauges were also installed in the transverse direction at the top of the slab over the girders (3.16). In all of these sections, strain gauges were installed in the reinforcing steel at the locations were flexural cracking was expected, i.e., the tensioned zone of the girder or slab.

In order to install the strain gauges on the reinforcing steel of the structure, the concrete cover had to be removed, the reinforcement surface was then sanded and cleaned for application of the strain gauge. For the gauges located at the top surfaces of the structure (E3, E6, etc...), the asphalt layer and concrete cover (in the case of sections S2 and S4) were also removed for the installation, as shown in Figure 3.17. The strain gauges were fixed to the structure using a cyanoacrylate adhesive. An epoxy layer was applied over the installed gauge for protection against environmental factors and for isolation of the soldered wire connections (3.18).

Signal cables were run from the sensor locations to the data acquisition system installed in a van under the span between columns P5 and P6. The data acquisition system used was the National Instruments SCXI. The system is assembled in modules according to the type of sensors being used in the instrumentation. A data acquisition rate of $100 \mathrm{~Hz}$ 


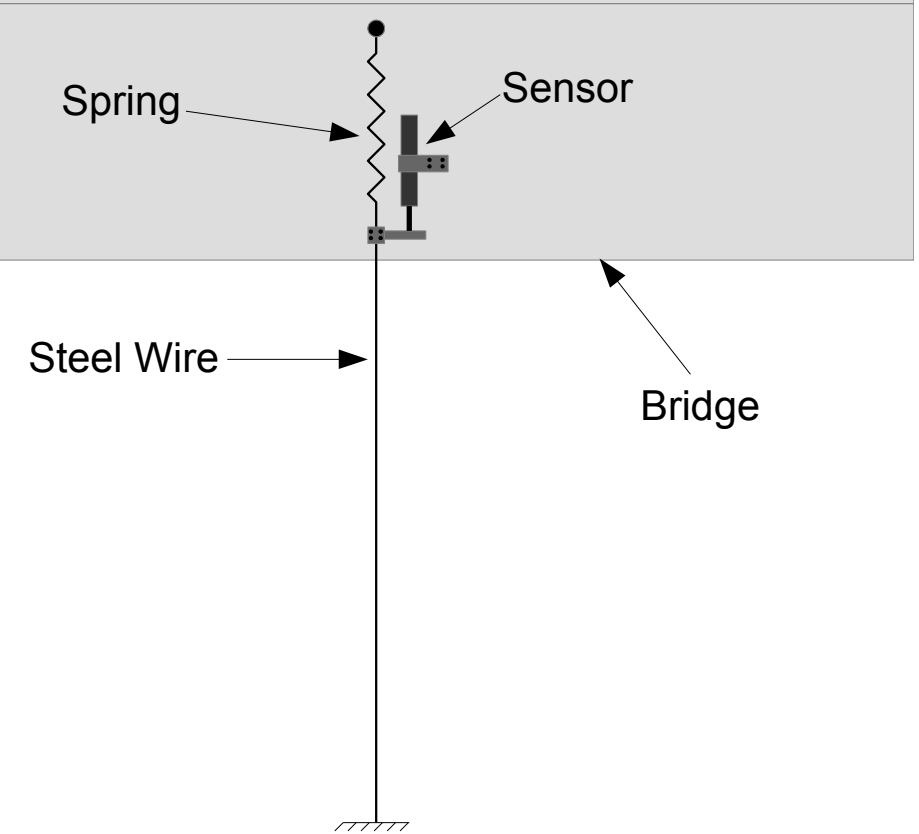

Figure 3.14.: Installation technique for measuring displacements at the midspan.

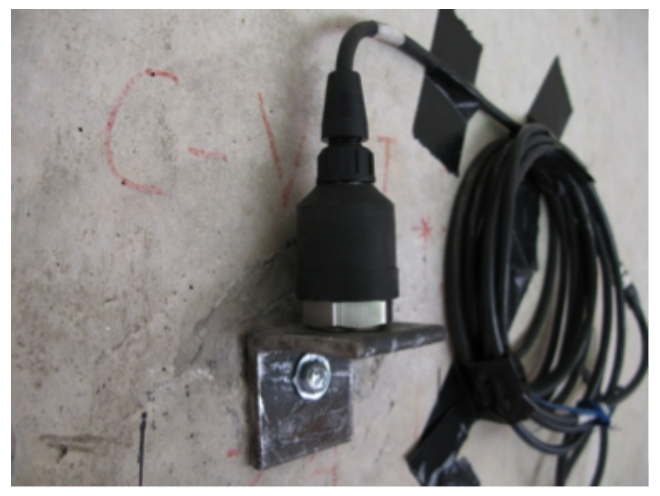

Figure 3.15.: Accelerometer installed at the side of the longitudinal girder.

was used during the monitoring. 

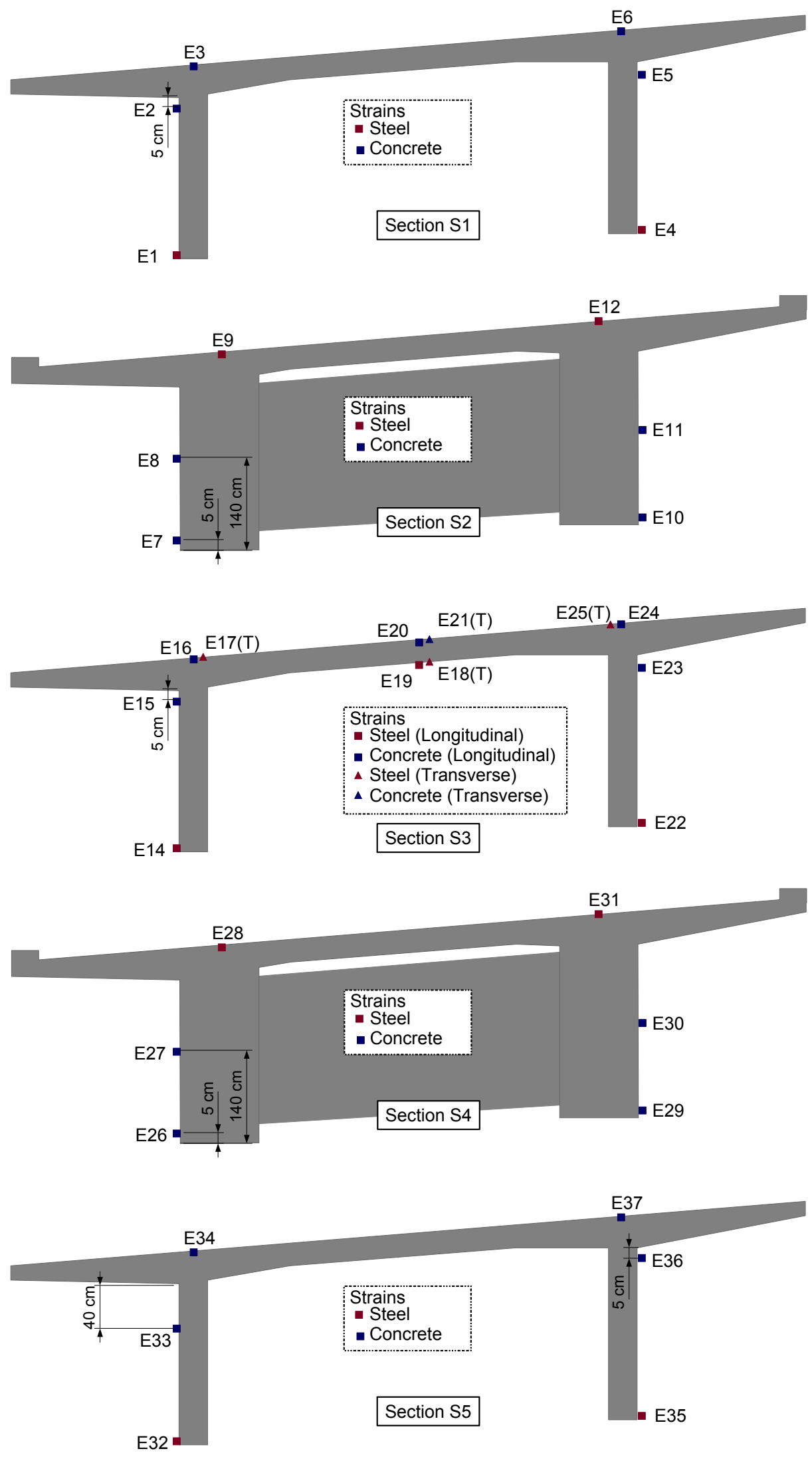

Figure 3.16.: Positioning of the strain gauges, in the analyzed cross-sections, for the short-term monitoring. 


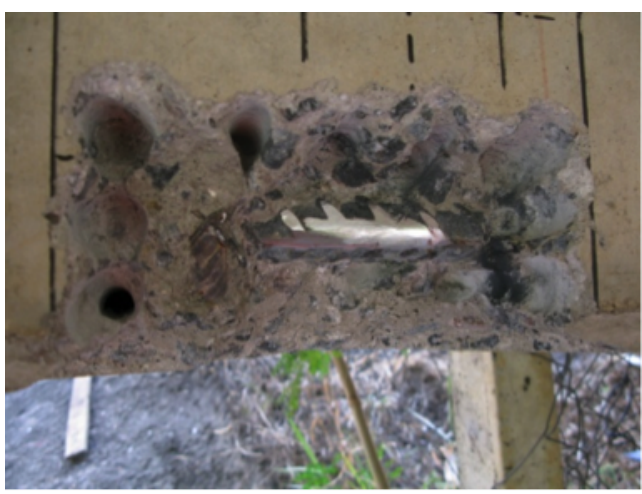

(a)

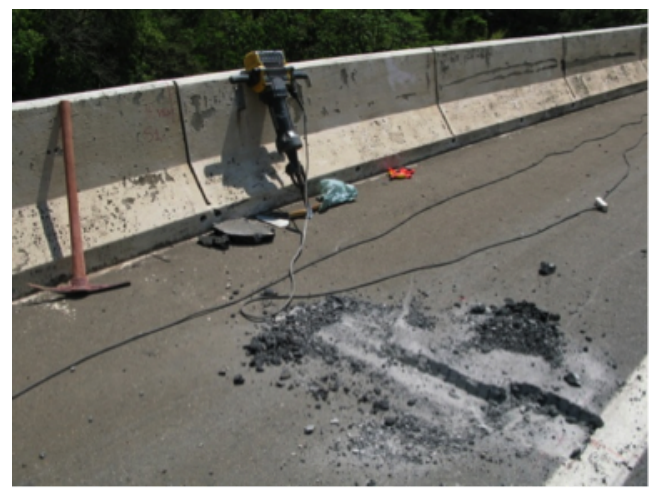

(b)

Figure 3.17.: Details showing: the uncovered reinforcement with the treated surface for strain gauge application (a); asphalt removal at the top surface for gauge installation (b).

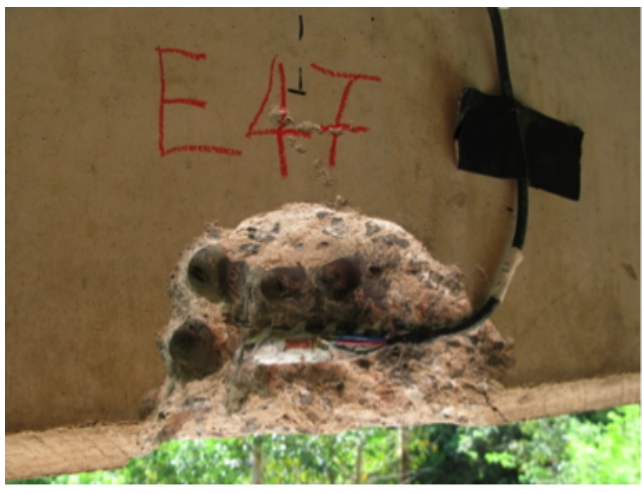

(a)

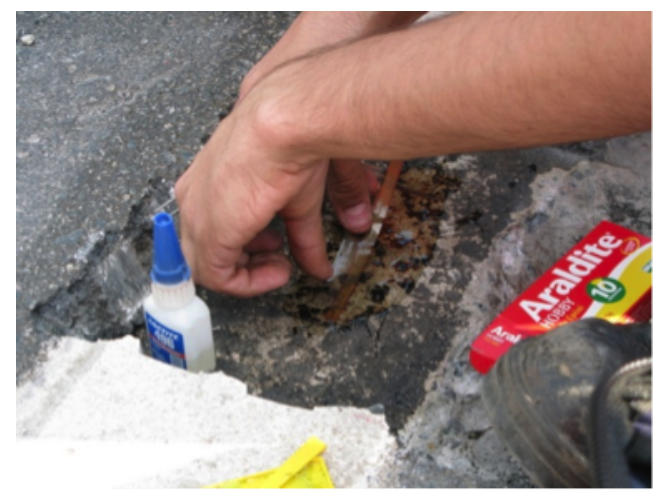

(b)

Figure 3.18.: Installed strain gauge in the bottom reinforcement (a), and installation of strain gauge at the top of the girder (b).
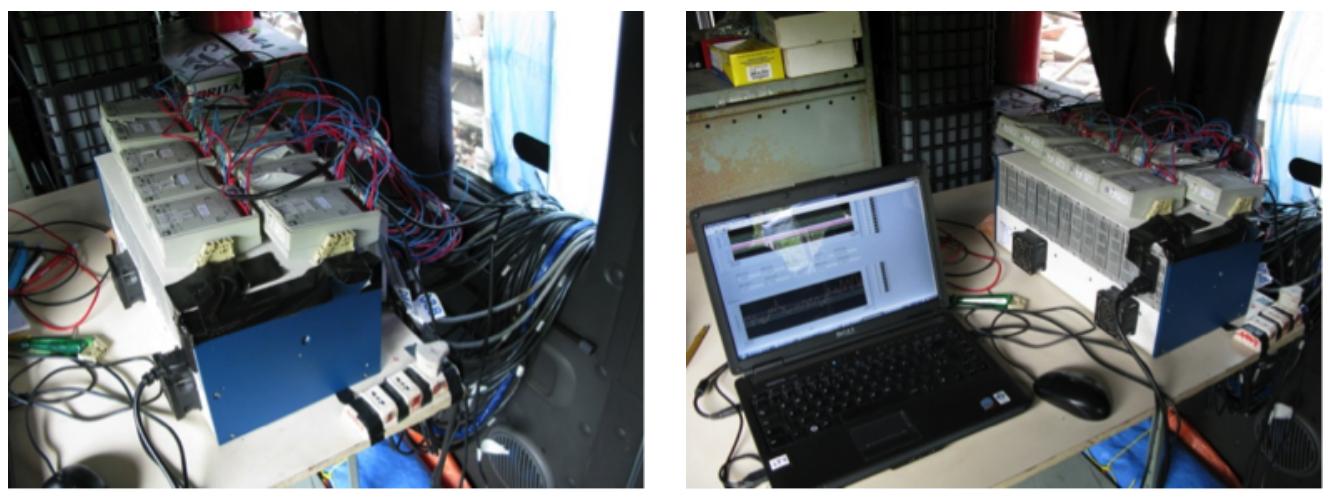

Figure 3.19.: Images showing the data acquisition setup. 


\subsubsection{Short-Term Monitoring and Load Testing}

The instrumentation of the bridge was followed by the short-term monitoring and load testing. Monitoring of the structural response due to uncontrolled traffic was carried out at various different time intervals between October 11-18, 2011. These samples were collected in 5 to 6 hour intervals at different periods of the day. The total of 18 hours of bridge uncontrolled traffic response was collected.

On October 19th, 2011, load tests, with the vehicle shown in Figure 3.20 (a), were performed on the structure. The test vehicle weighed a total of 44.3 metric tons and had the axle configuration shown in Figure 3.20 (b). A set of three crawl tests were done with the vehicle passing at different lanes:

- Static Test 1 - passage in the innermost lane (Figure 3.21 (a));

- Static Test 2 - passage in the longitudinal center line (Figure 3.21 (b));

- Static Test 3 - passage in the outermost lane (Figure 3.21 (c)).

During each passage the truck driver was asked to move at a maximum speed of $10 \mathrm{~km} / \mathrm{h}$. Once the truck reached each of the instrumented cross sections, it stopped and waited for around $30 \mathrm{~s}$ to allow for the signals to stabilize.

For the dynamic tests the test vehicle passed in the innermost lane at the speeds of 10 $\mathrm{km} / \mathrm{h}, 40 \mathrm{~km} / \mathrm{h}, 60 \mathrm{~km} / \mathrm{h}$, and $90 \mathrm{~km} / \mathrm{h}$.

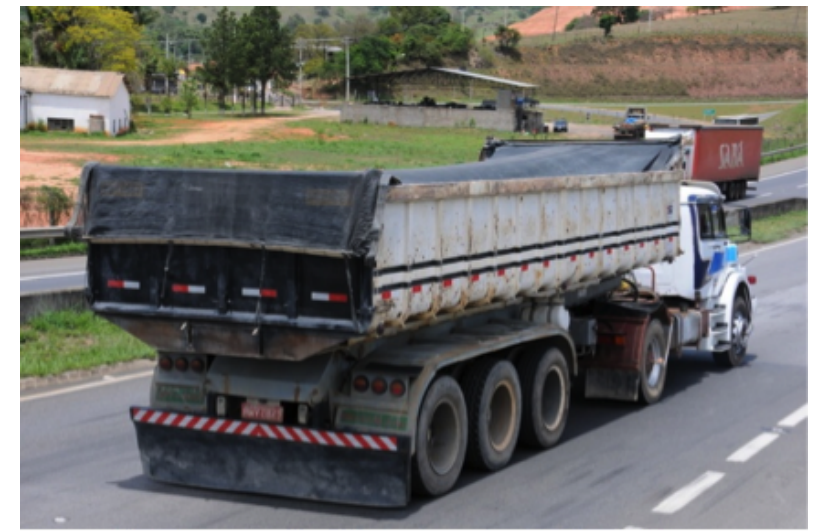

(a)

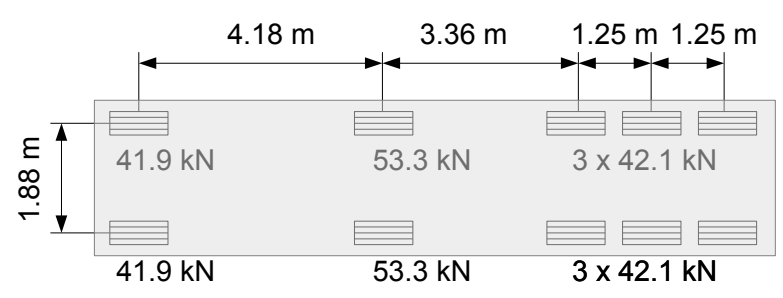

(b)

Figure 3.20.: Test vehicle that was used (a); and its axle load configuration (b). 
3.2 Visual Inspection, Testing, and Short-Term Monitoring

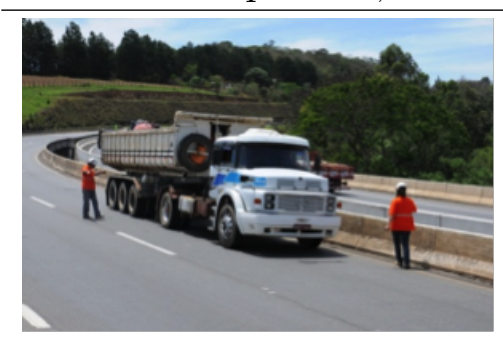

(a) Static Test 1

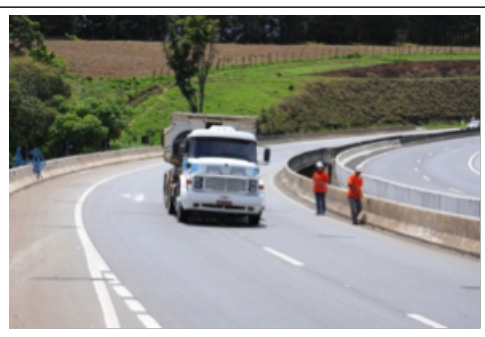

(b) Static Test 2

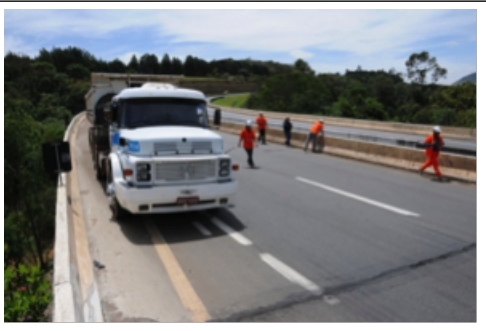

(c) Static Test 3

Figure 3.21.: Picture showing the truck position for the series of static tests.

\subsubsection{Results from Short-Term Monitoring and Testing}

A brief overview of the collected data is shown below. For brevity, only a part of the collected data will be shown in this section, a compendium of this data can be found in Appendix A.

\subsubsection{Static and Dynamic Testing}

To summarize the comparison between the results obtained in the quasi-static and dynamic tests, a procedure based on peak picking was used that aligns the signals of the different tests. The resulting graphs are equivalent to changing the $\mathrm{x}$-axis from time to load position, i.e., every $\mathrm{x}$-axis value corresponds to a specific truck position.
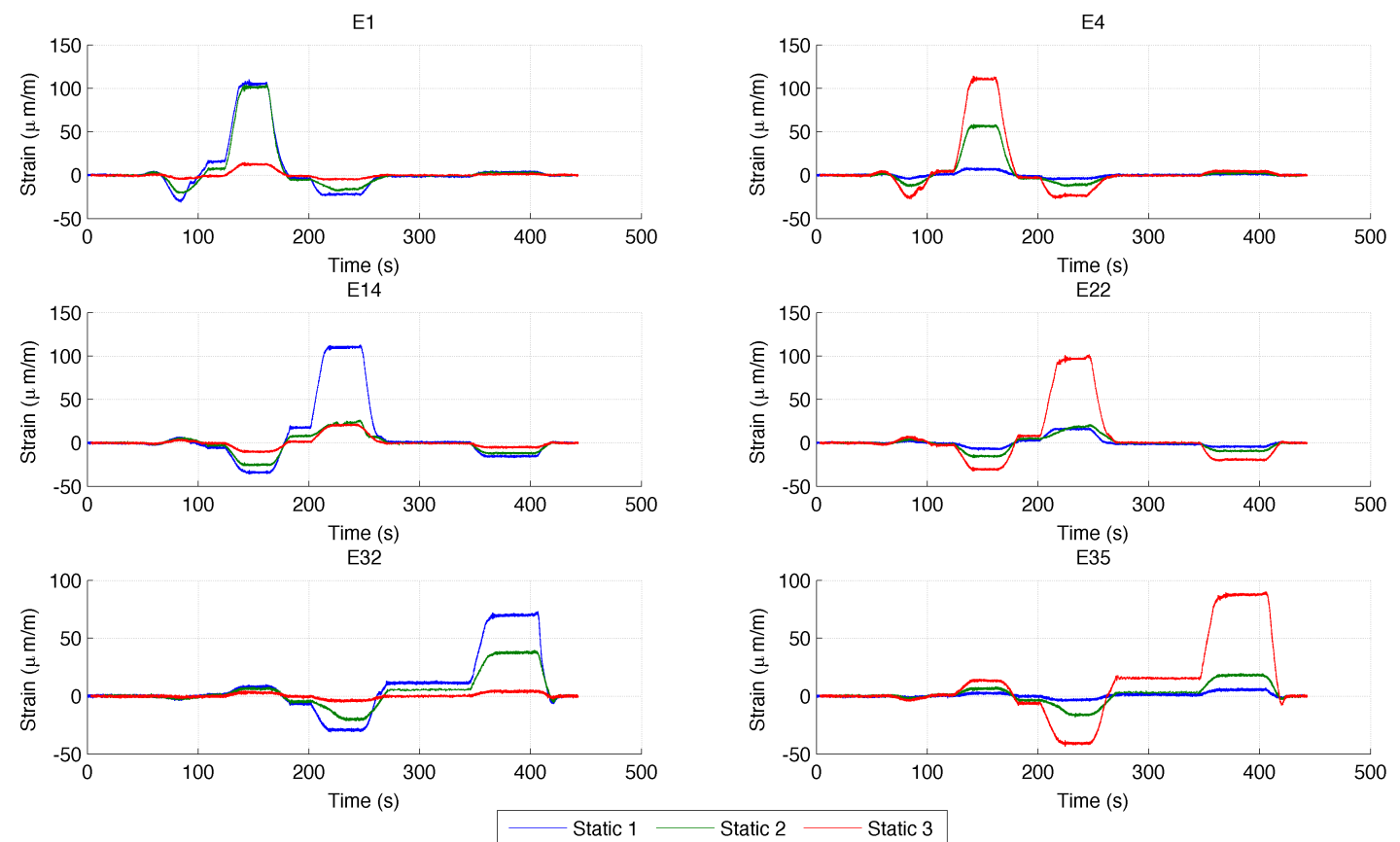

Figure 3.22.: Aligned strain time-histories, of selected sensors in the girder, for the three static tests.

The results from the six strain gauges located at the bottom reinforcement of the longitu- 
dinal girders are shown in Figure 3.22. For the most part, the results are consistent with the expected behavior. Showing larger values for E1, E14, and E32 for Static Test 1 and larger values for E4, E22, and E35 for Static Test 3, which is expected since the truck is over the respective girders in each case.
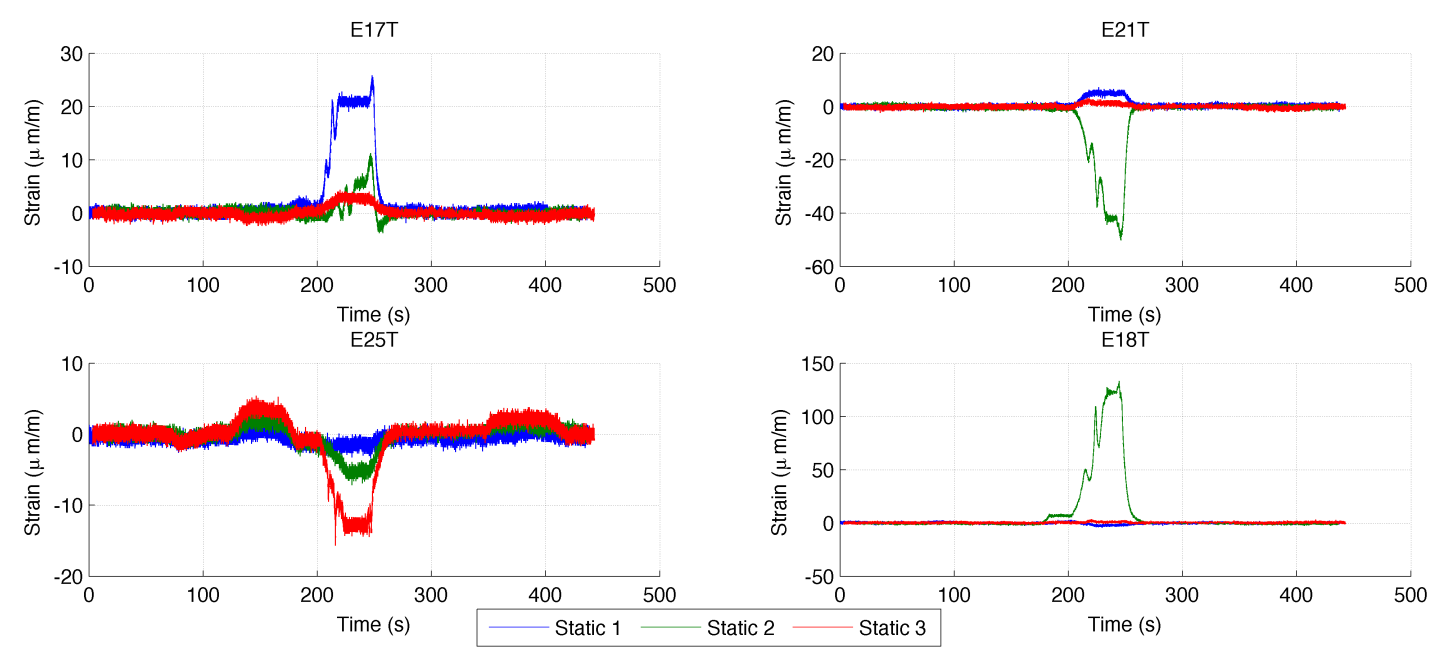

Figure 3.23.: Aligned strain time-histories, of selected sensors in the transverse direction of the deck, for the three static tests.

Figure 3.23 shows the aligned signals for the sensors installed at the deck on section S3. The results show considerable flexure occurring for test 2, which occurs when the truck is on the center of the deck slab. For the other two tests no significant changes in flexure appear at the center of the slab. However, for sensors E17T and E25T located where the slab joins the girder, changes are registered for tests 1 and 3. In these cases it would be expected that tensile strain were to occur, however, due to the superelevation more complex stress distributions may play a part on the recorded signals, as seen in channel E25T.

The same alignment procedure was applied to the dynamic tests, additionally the alignment also considered the Static Test 1, since it was in this lane that the dynamic tests were carried out. In the same manner as for the static tests, Figure 3.24, shows the results from the sensors located at the bottom reinforcement of the longitudinal girders. These results show that for the test vehicle used and speeds tested, some changes were detected between the dynamic and static cases. In the case of sensors E14 and E32, the static response was higher than the dynamic, this is most likely due to the fact that, at higher speeds, the driver was not able to stay as close to the inner concrete barrier as was done in the static case. For the sensors located in the outer girder, there are some levels of amplification during the higher speed runs. However, it is difficult to state that this is due to dynamic factors or due to mispositioning of the test vehicle. The results shown in Figure 3.25 demonstrate that the hypothesis of mispositioning is probable: there is considerably more flexure in the center of the deck slab for the $90 \mathrm{~km} / \mathrm{h}$ case than for the 
other cases.
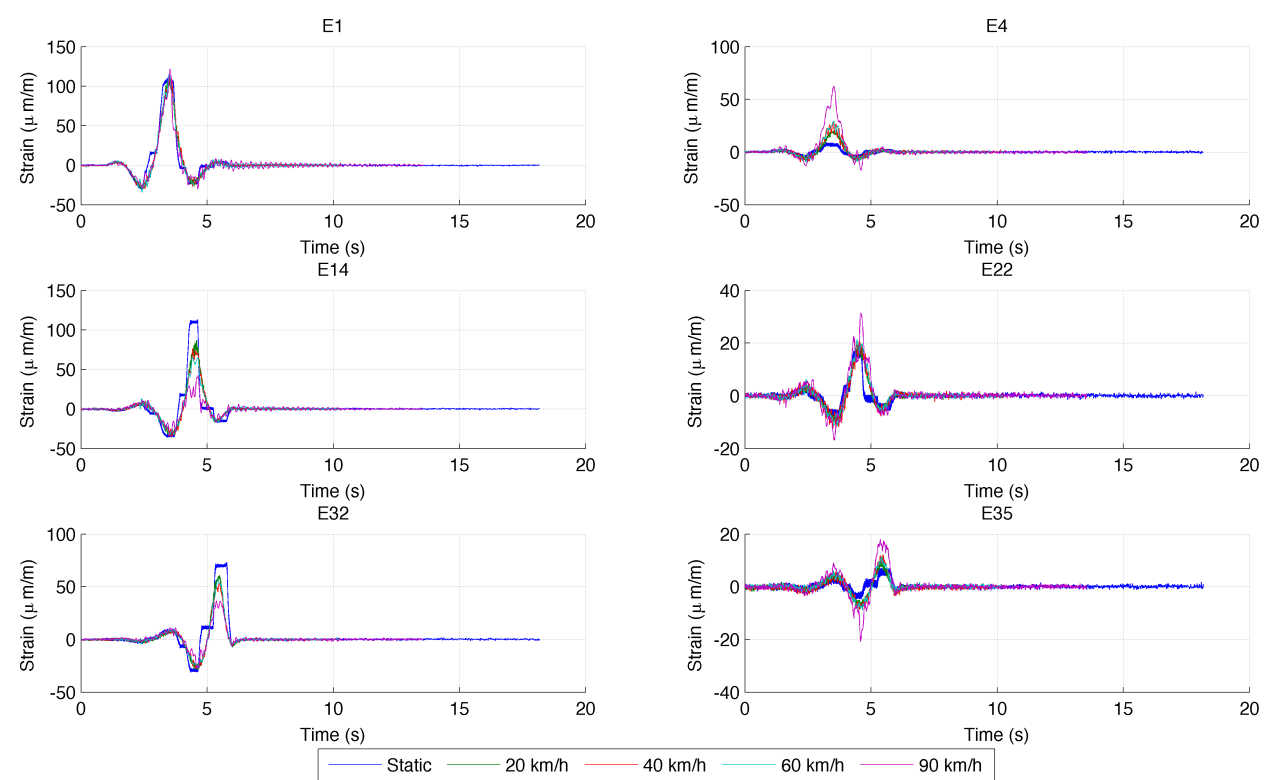

Figure 3.24.: Aligned strain time-histories, of selected sensors in the girders, for the dynamic tests.
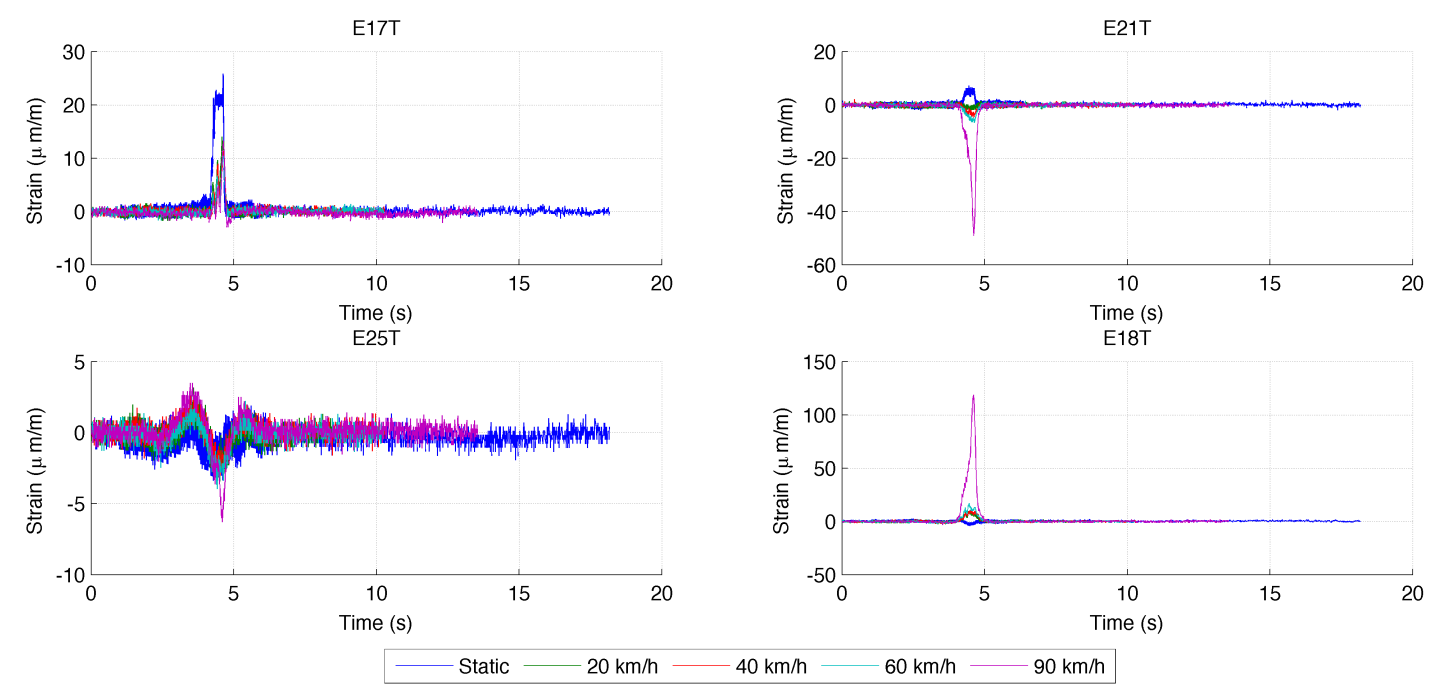

Figure 3.25.: Aligned strain time-histories, of sensor in the transverse direction of the deck, for the dynamic tests.

\subsubsection{Short-Term Monitoring Results}

During the short-term monitoring 18 hours of response time-histories were collected. A sample of this data is shown in Figure 3.26; it can be seen that, each peak seen in that chart corresponds to a loading event. These time histories were later processed, using a digital trigger approach, where the individual loading events were extracted. These individual loading events, which correspond to a single heavy vehicle passage or a combination of 
vehicles over the bridge, were then processed for maximum and minimum values. This allowed for the creation of a database of maximum and minimum strains due to loading events.

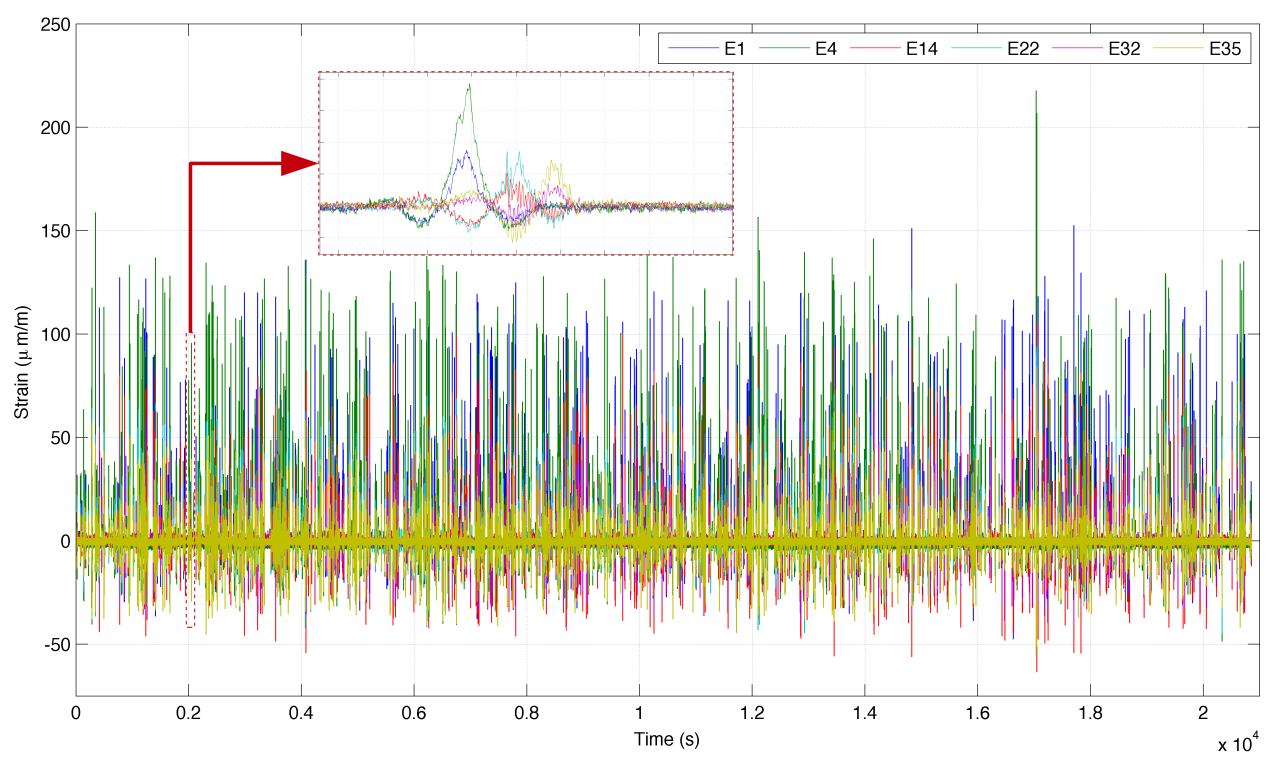

Figure 3.26.: Example of collected data in the short-term monitoring.

Strains at the bottom reinforcement of the longitudinal girders at the midspans (E1, E4, E14, E22, E32, and E35) were used for the digital trigger. The signals were analyzed in segments of 100 samples, and once the peak to peak values reached $25 \mu \mathrm{m} / \mathrm{m}$ the trigger was set off. Once the trigger is set, the trigger subsample and the following subsamples are stored until the peak to peak values fall below $15 \mu \mathrm{m} / \mathrm{m}$.

Figure 3.27 shows the histograms of the maximum strains, obtained from the 3,055 registered loading events, for the trigger channels. The shape of these histograms are dependent on the traffic characteristics, as well as on the load distribution between the two girders. Since the bridge has two main traffic lanes and most of the heavy vehicles stay on the right lane, which distributes more load to the outer girder than the inner, it is expected that the histograms for the inner girders show two modes. One with lower strain intensities, which corresponds to the partial loading due to heavy traffic on the right lane as well as any concurrent vehicle that may have passed in the left lane during the loading event. The other mode, with greater strain intensities, corresponds to the passage of heavy vehicles on the left lane. These tendencies can be noticed in histograms for E1 and E32.

It is possible to notice similarities in the shapes of the histograms for E4, E22, and E35 which are located in the outer girder of the bridge. The mode located in the region with greater strain intensities shows larger frequencies than reported for the inner girder sensors. This again, is explained due to the fact that heavy traffic tends to stay on the right lane. 
3.2 Visual Inspection, Testing, and Short-Term Monitoring

For comparison, Table 3.2 shows the maximum and minimum values obtained for the strain sensors during the 18 hours of monitoring. Relatively low strain values were registered at sections S2 and S4, which are located over the supports. In a continuous beam, these regions are usually stiffer and, therefore, the flexural moments at these sections should be higher. Two factors can be attributed to these results: one is the fact that the analyzed sections were located exactly over the supports where a transverse beam is located. The presence of the transverse beam creates a much greater local stiffness resulting in very little strains. If the analyzed section was moved immediately before or after the transverse beam, these strains would be considerably higher. The other factor that contributes to the smaller values is that, at these regions, the girders have $1.0 \mathrm{~m}$ in width. This increased stiffness also contributes to the low strain values that were recorded.

\begin{tabular}{|c|c|c|c|c|c|c|c|c|c|c|c|c|c|}
\hline Section & Sensor & Max & Min & Sensor & Max & Min & Section & Sensor & Max & Min & Sensor & Max & Min \\
\hline \multirow{3}{*}{ S1 } & E1 & 179.0 & -55.8 & E4 & 217.8 & -55.4 & \multirow{3}{*}{$\mathrm{S} 2$} & E7 & 10.6 & -35.2 & $\overline{E 12}$ & 19.7 & -39.4 \\
\hline & $\mathrm{E} 2$ & 7.9 & -3.9 & E5 & 10.1 & -7.4 & & $\mathrm{E} 8$ & 4.5 & -8.1 & E11 & 6.1 & -8.4 \\
\hline & E3 & 20.1 & -36.7 & E6 & 9.9 & -28.5 & & E9 & - & - & E10 & 43.5 & -11.8 \\
\hline \multirow{3}{*}{ S3 } & $\overline{\text { E14 }}$ & 136.5 & -67.5 & $\overline{\mathrm{E} 22}$ & 109.7 & -47.3 & \multirow{3}{*}{ Deck } & 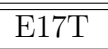 & 25.7 & -6.2 & $\overline{\text { E20 }}$ & 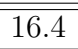 & "-50.0 \\
\hline & E15 & 14.6 & -20.3 & E23 & - & - & & E18T & 266.3 & -8.4 & E21T & 9.9 & -110.0 \\
\hline & E16 & 24.2 & -32.1 & E24 & 29.4 & -9.2 & & E19 & 64.5 & -41.4 & E25T & 7.8 & -20.5 \\
\hline \multirow{3}{*}{$\mathrm{S} 4$} & $\overline{\mathrm{E} 26}$ & 11.4 & -25.2 & $\overline{\mathrm{E} 29}$ & $\bar{~} 12.9$ & -32.1 & \multirow{3}{*}{ S5 } & $\overline{\text { E32 }}$ & 86.6 & -47.3 & $\overline{\text { E35 }}$ & 84.3 & -58.9 \\
\hline & E27 & 5.6 & -11.0 & E30 & 13.3 & -14.4 & & E33 & 9.8 & -8.3 & E36 & 6.4 & -6.0 \\
\hline & E28 & - & - & E31 & 33.1 & -14.8 & & E34 & 9.5 & -22.0 & E37 & 40.7 & -63.7 \\
\hline
\end{tabular}

Table 3.2.: Maximum and minimum recorded strains during the short-term monitoring.

The highest strain value was found for sensor E18T which is a strain gauge installed in the transverse bottom reinforcement of the deck. This result is compatible with what was seen in the load testing phases. The second largest strain value was found in sensor E4 which is the bottom gauge in the outer longitudinal girder of the $30 \mathrm{~m}$ span. The values are all within what is expected for service loads for this type of structure.

As mentioned, these results are compiled from a collection of time-history response data. This data was collected in 5 to 6 -hour intervals at different times of the day and on different days. Therefore, these results are not capable of properly characterizing the traffic load condition of the structure. They are able to demonstrate, however, that the bridge response is compatible with a structure in good service condition. In the following section, the long-term monitoring project of the Jaguari River Bridge will be shown. This system allows for the characterization of the bridge traffic loading, as well as the behavior due to environmental factors. 

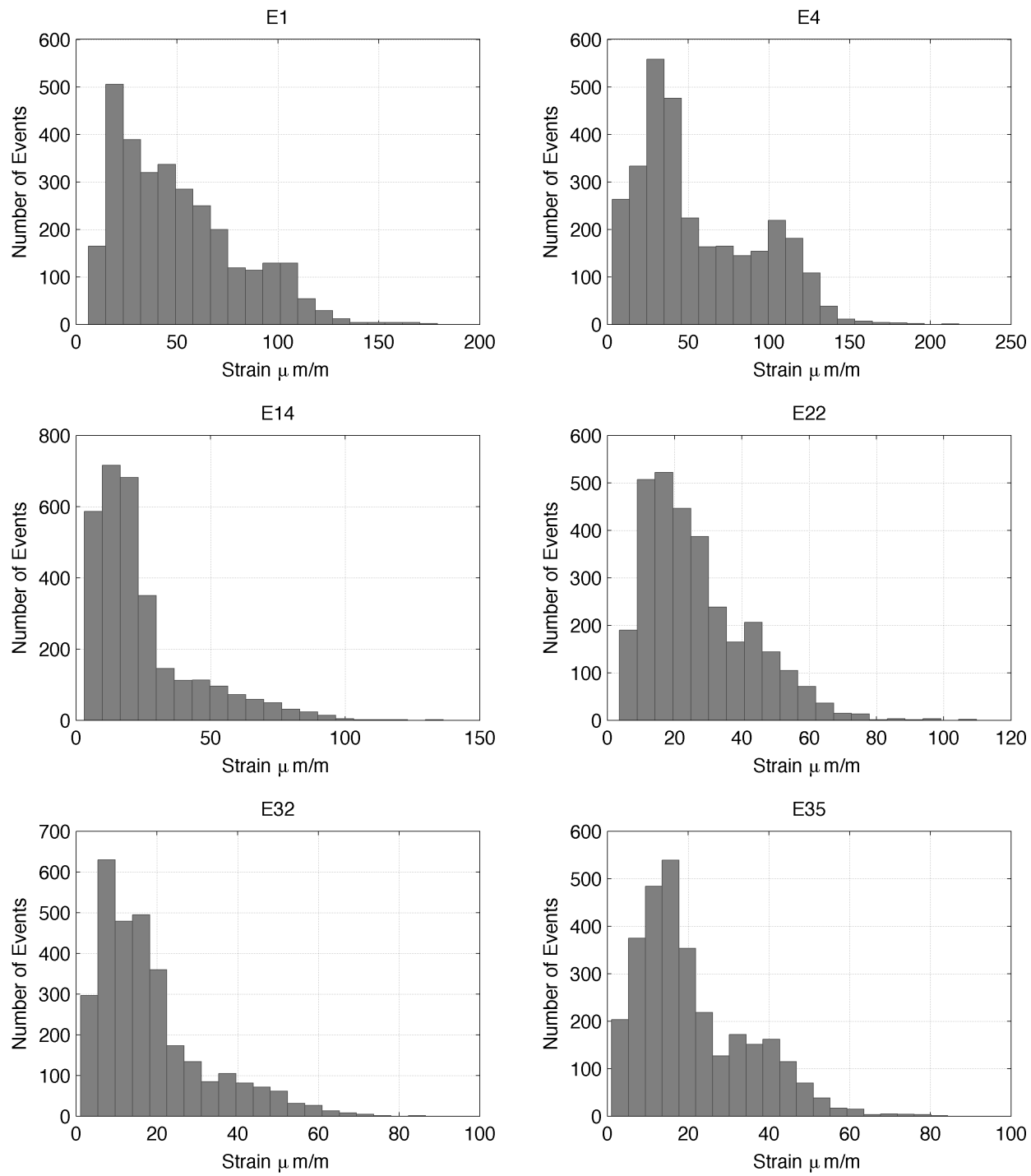

Figure 3.27.: Histograms of maximum strains obtained in the trigger channels for the collected short-term data.

\subsection{Long-Term Monitoring System}

As mentioned earlier, the developed monitoring system was aimed at creating a prototype for SHM. Therefore, two measurement systems were installed at the bridge site:

- Quasi-Static Monitoring System - this equipment has the objective of measuring the changes in response due to temperature variations and degradation;

- Dynamic Monitoring System - this monitoring scheme has the objective of measuring fast changing phenomenons such as bridge live-loads.

In practice, depending on the specific objectives of the application, one or both of these systems might be more adequate for use. The adopted setup is of greater research value since it establishes a testbed for measurement and data analysis techniques. In the next subsections an overview of the system is given. 


\subsubsection{Sensors}

Most of the sensors that are used for short-term monitoring may be used in long-term applications. In general two factors are usually taken into consideration when selecting sensors for long-term applications: signal stability and durability. For instance, some strain transducers that are used for short tests may present strong signal variations due to temperature. Therefore, their usage for long monitoring periods is limited. The durability issue is evident since the sensor and its fixtures must be in good state during the monitoring system lifetime.

Vibrating wire transducers are widely used in geotechnical long-term monitoring applications due to its signal stability and durability. The measurement principle is based on the fact that a change in the stress condition of a tensioned steel wire results in changes in its resonance frequency. This allows for taking measurements of physical quantities, such as strains and displacements, through a high-level frequency signal. This high-level signal is less prone to variations due to ambient noise and changes in cable temperature. The use of this technology in structural monitoring, however, is limited to cases in which the observed phenomenons are slowly changing. This is due to the fact that, for each reading, a coil which induces free vibration to the steel wire, must be enabled, and its vibration frequency read. This process takes a fraction of a second, depending on the equipment, and, therefore, cannot be used for applications which demand, for example, more than one reading per second. For the installed Quasi-Static Monitoring System, where a reading was taken once every 15 minutes, this type of sensor is very adequate.

For the Dynamic Monitoring System, in which reading were taken at $100 \mathrm{~Hz}$, resistive based sensors were used. As will be shown in detail, electrical strain gauges and full-bridge strain transducers were used for strain measurements and potentiometric displacement sensors were used for relative displacement measurements.

The instrumentation plan for the long-term monitoring system follows, approximately, the instrumentation plan shown in section 3.2.3.1. However, some changes were made due to budget and installation time constraints. As shown in Figure 3.28, displacements were measured at the midspans at sections S3 and S5 under each girder. The sensor installation was done using the same scheme, shown in Figure 3.14, that was used in the short-term monitoring. Displacements were not measured at S1, as done in the short-term monitoring, due to the impossibility of establishing a fixed point of reference due to the river.

At each displacement measurement point two different sensors were installed: a potentiometric and a vibrating wire transducer (Figure 3.29(a)). Longitudinal, transverse and vertical displacements were measured at the columns P4B, P5B, and P6B. An apparatus, shown in Figure 3.29(b), was fabricated to install these sensors. The moving ends of the 


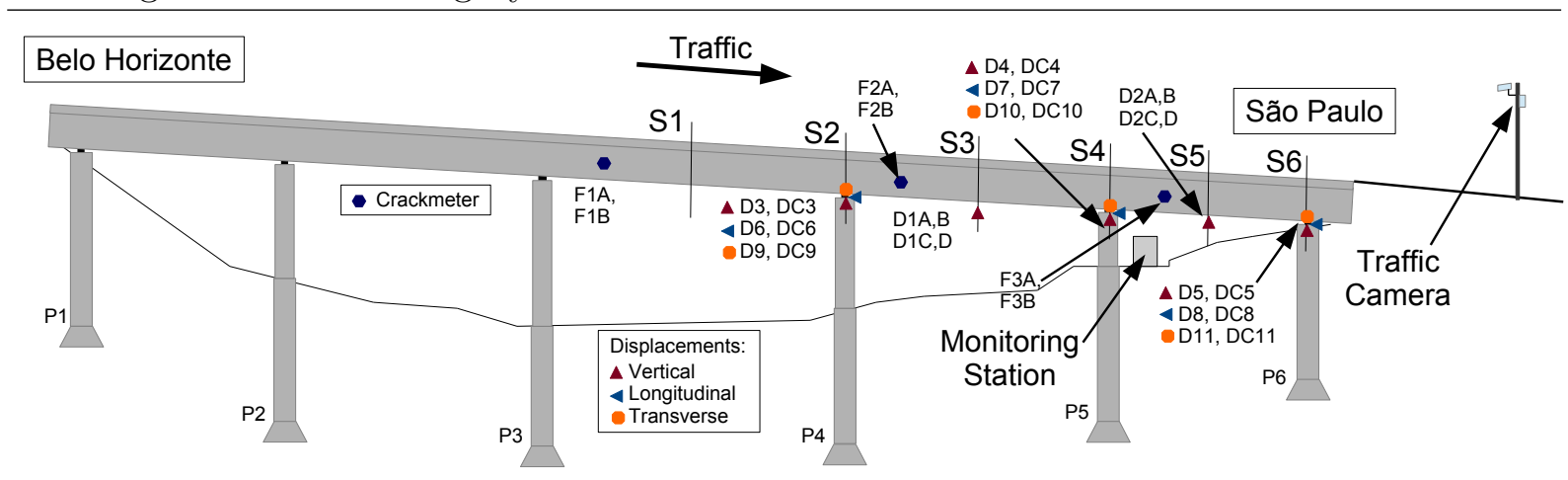

Figure 3.28.: Longitudinal view of the long-term monitoring system for the Jaguari River Bridge.

sensors were linked to the structure using thin steel cables which prevented bending on the bodies of the sensors due to lateral movements.

Vibrating wire crackmeters were installed in some of the cracks that were found along the longitudinal girder. Crackmeters are displacement sensors specially designed to monitor the evolution crack openings. The sensors installed in these girders have a full range of 4 $\mathrm{mm}$. As shown in Figures 3.28 and 3.30, these sensors were positioned close to columns P3, P4, and P5. These cracks were detected during the first visual inspections and were later treated with epoxy. However, monitoring was chosen in order to follow the progress of these cracks.

A modified vibrating wire displacement transducer was also used to monitor the progress of flexural cracking in the deck slabs. This $3 \mathrm{~mm}$ full scale displacement transducer, shown in Figure 3.31, is installed with a $75 \mathrm{~cm}$ base. The idea behind this sensor is to measure the total crack opening of any cracks that are present along this base. This total crack opening value may be divided by the base length to obtain the mean strain in that portion of the slab. Degradation in the slab may be detected trough monitoring of this output.

Strain measurements were taken at sections S1, S3, and S5, shown in Figure 3.28. Three measurement points were established along the height of each girder, the location of these points is shown in Figure 3.32. Again, in the locations in which flexural cracking was expected the strain measurements were taken on the reinforcement, elsewhere, the strain was measured at the concrete surface. At every measurement point a vibrating wire gauge was installed together with a resistive type sensor; this is illustrated in Figure 3.33.

In the long-term monitoring it was opted not to install gauges in the top surface of the structure. This was due to the difficulties in getting permission to work in the road surface since it required for openings to be made in the asphalt. Also, the sections over the supports were not instrumented with strain gauges as done in the short-term monitoring. This choice was made to simplify the instrumentation. It should be remembered that 


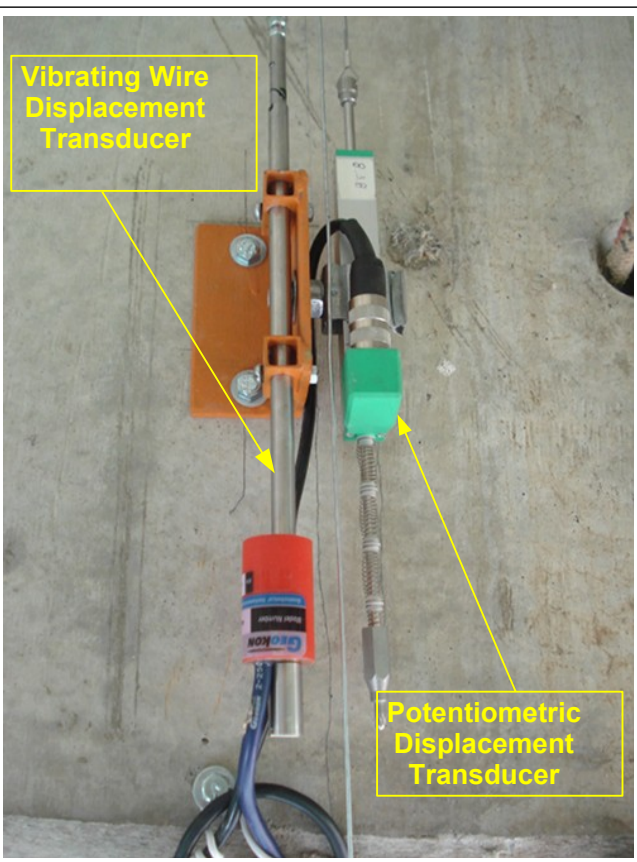

(a)

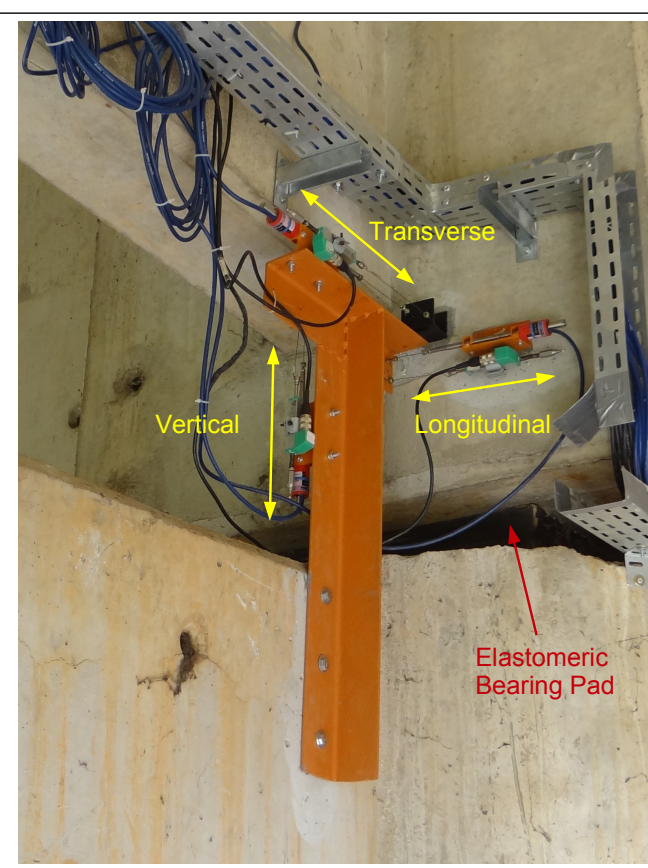

(b)

Figure 3.29.: Images showing details of the displacement transducers that were installed at the bridge site: (a) different displacement transducers used; (b) measurement scheme used to take measurements in three directions.

for the long-term monitoring each measurement point received two sensors and that the installation time of each one of these was considerably higher than the ones used for the short-term monitoring. Therefore this simplification was also done because of time constraints.

The vibrating wire strain gauges installed in the reinforcing steel were fixed to bases that were welded to the rebar. Electrical strain gauges were then bonded to the rebar, due to the long-term nature of the monitoring, the HBM 2-compound adhesive X60 was used for this purpose. For electrical isolation and physical protection, the installed sensors, seen in Figure 3.33(a), received an epoxy coating, for the electrical strain gauges, and a steel casing cover, for the vibrating wire gauge. The concrete cover opening, made for sensor installation, was later closed using a cement grout mix.

For the strain measurements in the concrete, full-bridge and vibrating wire strain sensors were used (Figure 3.33(b)). The full-bridge strain transducer is composed of four active strain gauges installed in a configuration that allows for the measurement of axial strain of the sensor body. These were fixed to the structure using anchors that were grouted into holes drilled in the structure. After hardening of the grouting material the sensors were fastened to the anchors using screws (vibrating wire) and nuts (full-bridge). 


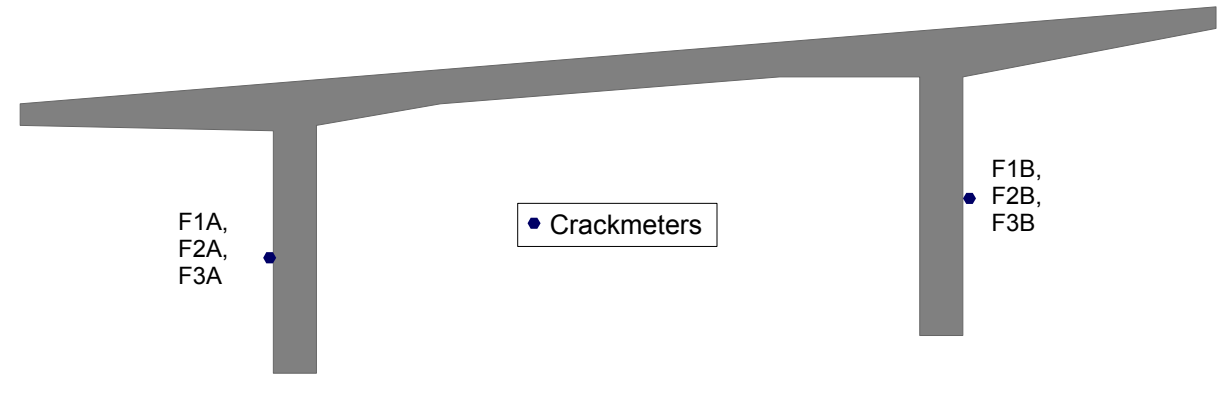

Figure 3.30.: Location of the crackmeters installed in the sides of the longitudinal girders.

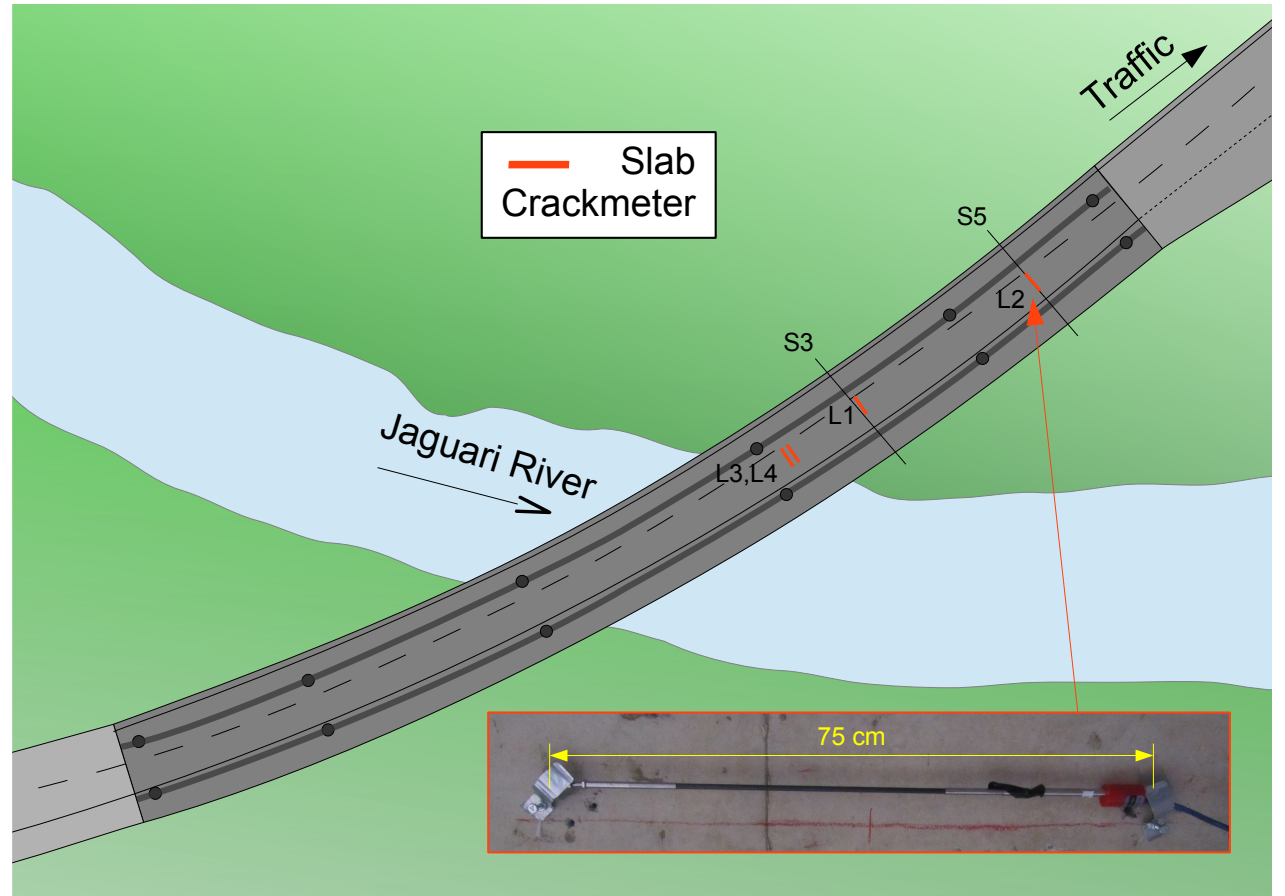

Figure 3.31.: Location of the crackmeters installed in the deck slab. 

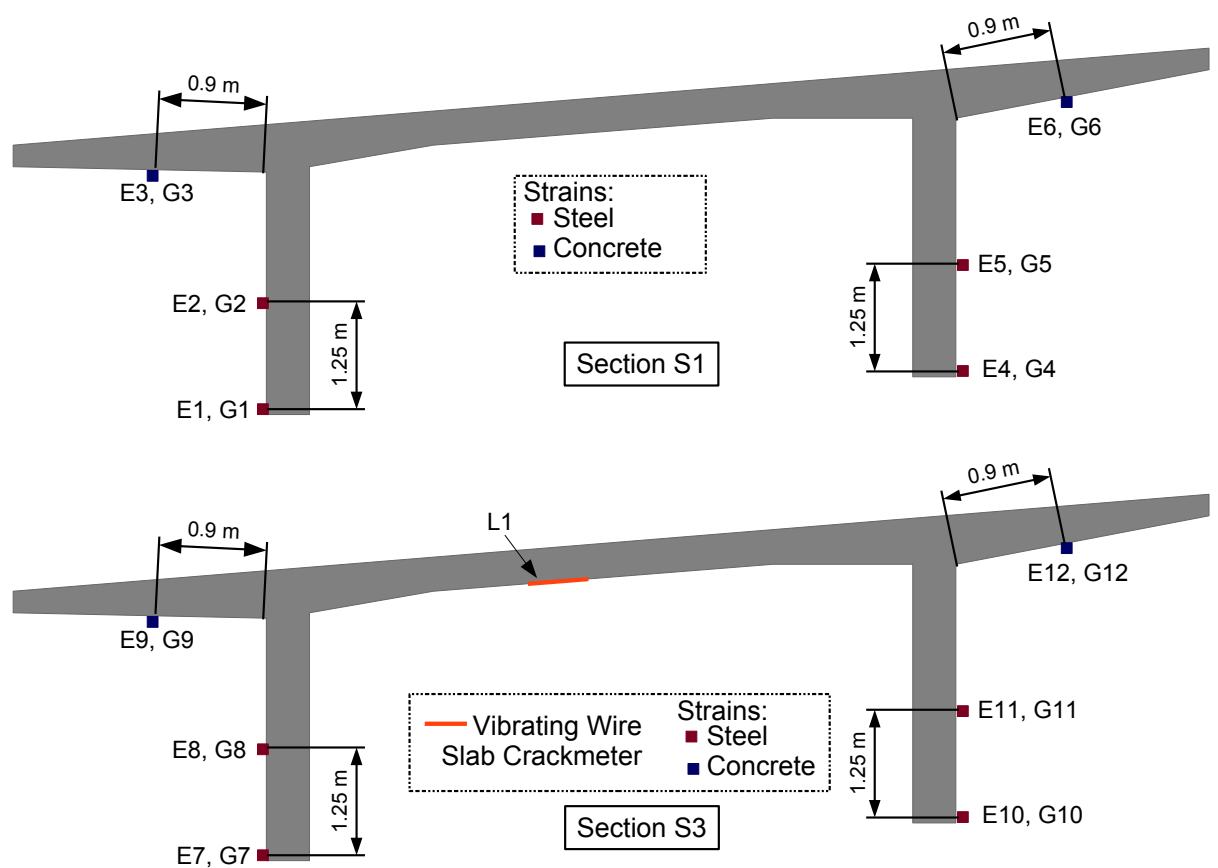

L2

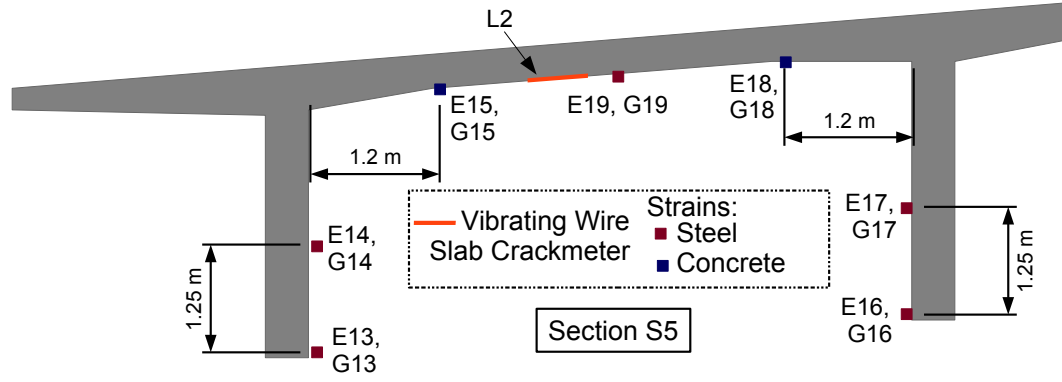

Figure 3.32.: Strain transducers installed in section S1.

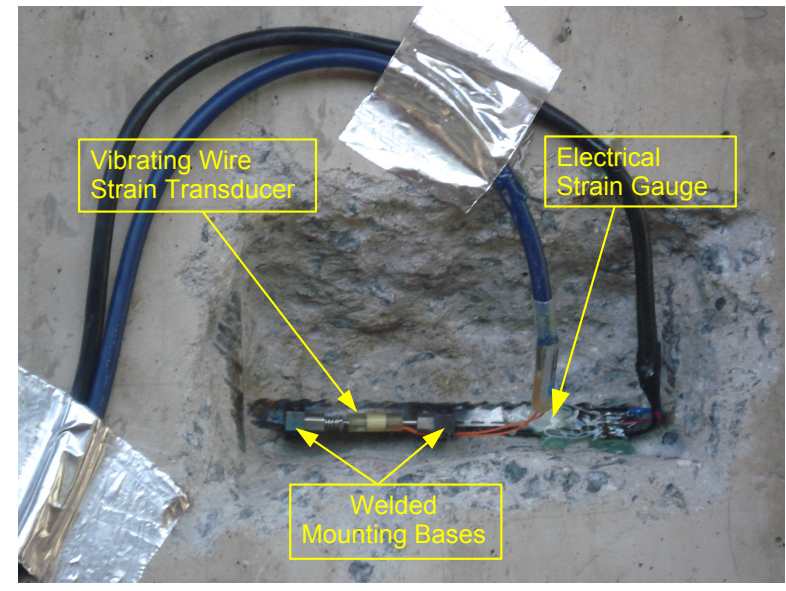

(a)

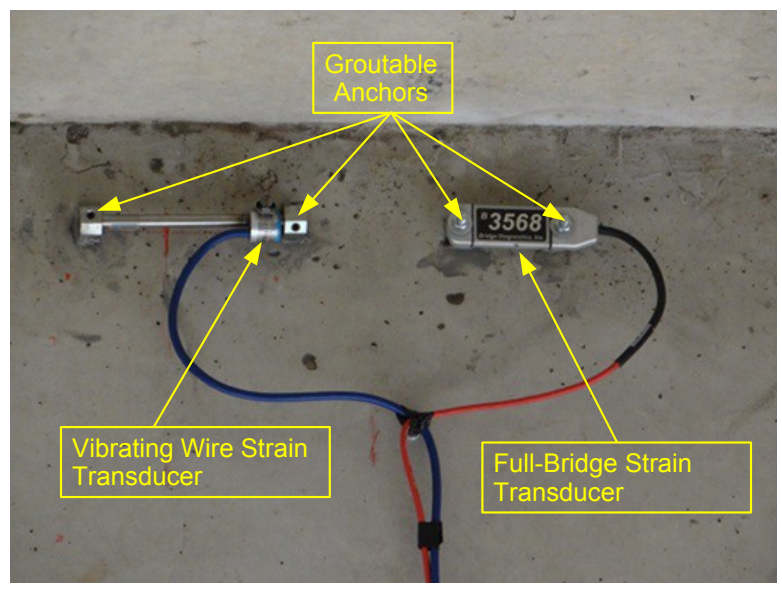

(b)

Figure 3.33.: Details showing the installation of the strain transducers in the reinforcement (a); and, on the concrete surface (b). 


\subsubsection{Data Acquisition and Logging Systems}

For the Quasi-Static Measurement System the Geokon MICRO-1000 datalogger was used. As mentioned the vibrating wire readings are made in terms of frequency readings. Internally this datalogger has a vibrating wire spectrum analyzer which is responsible for making these readings. The vibrating wire sensors, installed along the structure, are connected to multiplexers positioned at key points of the bridge. These multiplexers allow for the channeling of multiple sensors through a single signal wire. Internally, the datalogger sets which multiplexer channel it wants to read and initiates the reading of that particular channel using the vibrating wire spectrum analyzer. The measured results are then stored internally and made available for download through network protocols. Three 16-channel multiplexers were used, in this case, for the 42 sensors that were installed.

The datalogger was programmed to take readings of all channels in 15 minute intervals. To make sure that the structure is not under load during the readings, a signal from the Dynamic Measurement System indicating the load status of the bridge is checked. In case this signal indicates loading, the sensor reading is postponed until the structure returns to a unloaded state. It should be noted that loads that are lower than the trigger threshold may still influence the signals. However, as it will be shown in the data analysis, by taking reading at 15 minute intervals, the signal can be easily treated to remove outliers.

Due to the necessity of higher acquisition rates for the Dynamic Measurement System another datalogger was used for this case. The CompactRIO system from National Instruments (NI) was adopted. This equipment has a powerful controller and can be assembled using different modules, each one for a specific type of measurement. Quarter-bridge strain gauge, full-bridge, and voltage measurement modules were used in this setup. The controller was connected to a local access network (LAN) which allows for transferring the datalogging programs into memory, as well as, for status communication and data transfer.

Programming of the controller is done using the graphical programing language of LabVIEW. A program was developed for acquiring, applying digital filters, and saving the data based on a digital trigger. A response based digital trigger was developed, in which the detection of peak-to-peak values of $25 \mu \mathrm{m} / \mathrm{m}$ on signals from channels E1, E4, E7, E10, E13, or E16, resulted in data recording until the return of the signals to normal values. Additionally, the functionality of saving time frames from an IP camera, installed at the bridge to monitor the traffic, during the loading event was programmed. As previously mentioned, this system also indicated whether the structure is under load for the other datalogger. This is done through a digital output signal that is enabled during the period in which the trigger is set. The resulting data from the live-load monitoring system is a file with the recorded time-history and sequence of captured images corresponding to the loading event. It should be mentioned that, the recorded time history data and images 
allows for different types of analysis to be run, which is of special interest for academia since it can be used by other researchers with, different, specific purposes.

An internet connection was established at the site using a wireless antenna. This connection allowed for remote access to the installed dataloggers. An overview of the arrangement of the data acquisition system installed for the long-term monitoring system is shown in Figure 3.34.

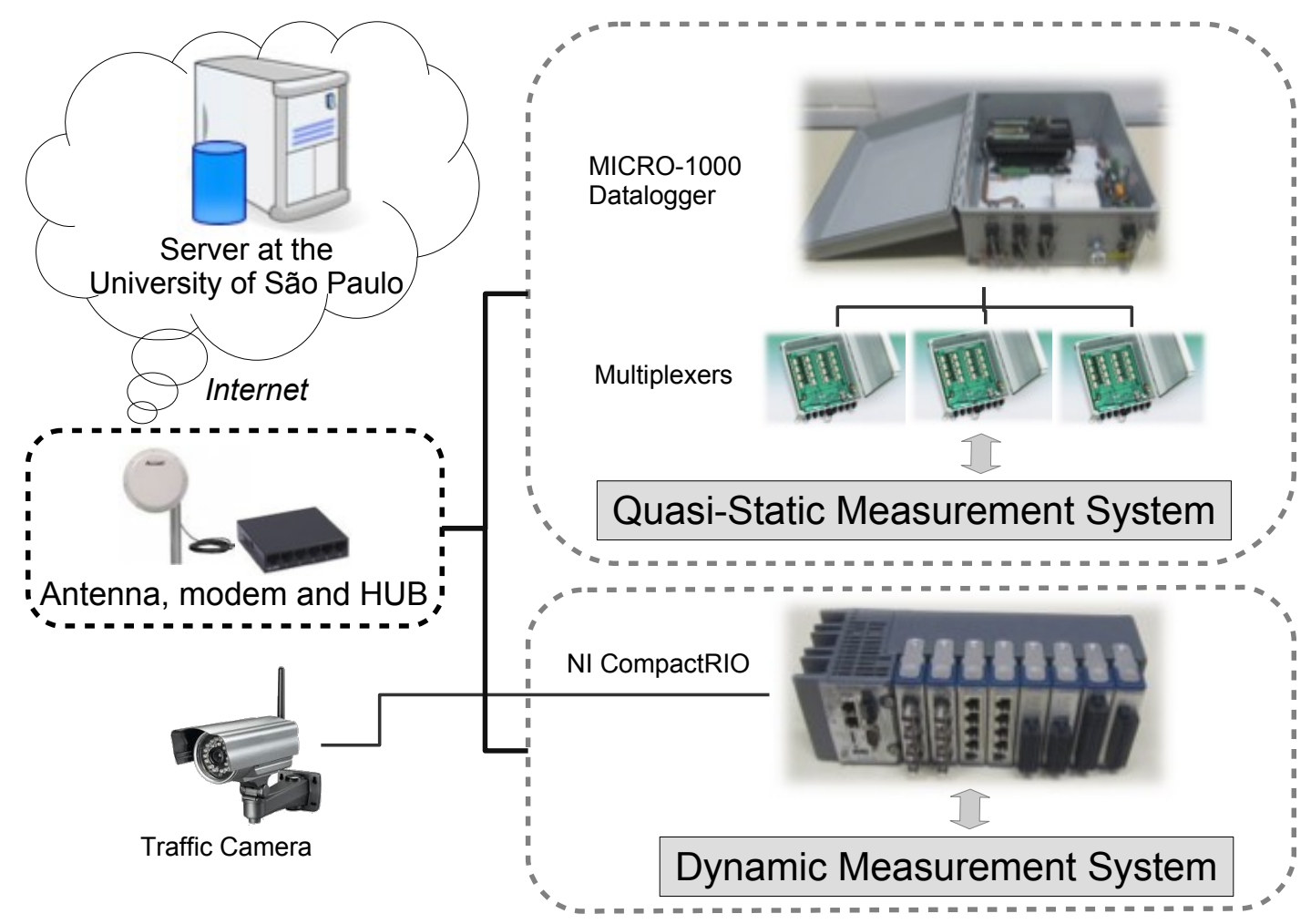

Figure 3.34.: Arrangement of the data acquisition systems used in the long-term monitoring of the Jaguari River Bridge.

\subsubsection{Data Management and Visualization}

Due to the large volume of data that ends up being generated when collecting live-load time history responses, in many cases, the collection of this data may not be feasible. However in this case due to the high-speed internet that was made available, it was possible to transfer this data for storage in computers located at the University of São Paulo. The generated files were made available through the built in FTP - File Transfer Protocol server of the CompactRIO controller. Using the, freely available, FTP client FileZilla these files were downloaded to the university computers. MATLAB scripts were written to process these files to remove temperature offsets and to obtain maximum and minimum values. 
For the vibrating wire sensor data the software Multilogger Suite was configured to automatically access the remote datalogger and download the newly available data. This software, which was also used for the datalogger programming, was configured to download the new data at every hour. This data is cumulatively stored in a comma separated text file. MATLAB scripts were developed to process the data file and analyze the data.

To be able to check the most recently collected data, as well as, its overall characteristics, a web visualization tool was used. The commercial software package Vista Data Vision was used for this purpose. This tool was configured to read the data file and create a database. It then continuously monitors the data file and adds new data to the database as it is found. A web interface was also configured to generate pages with graphs with the information stored in the databases. The user can then select a page and visualize these graphs and use the page tools to shift between different timeframes of the collected data. Figure 3.35 show a typical page that can be generated by accessing the website.

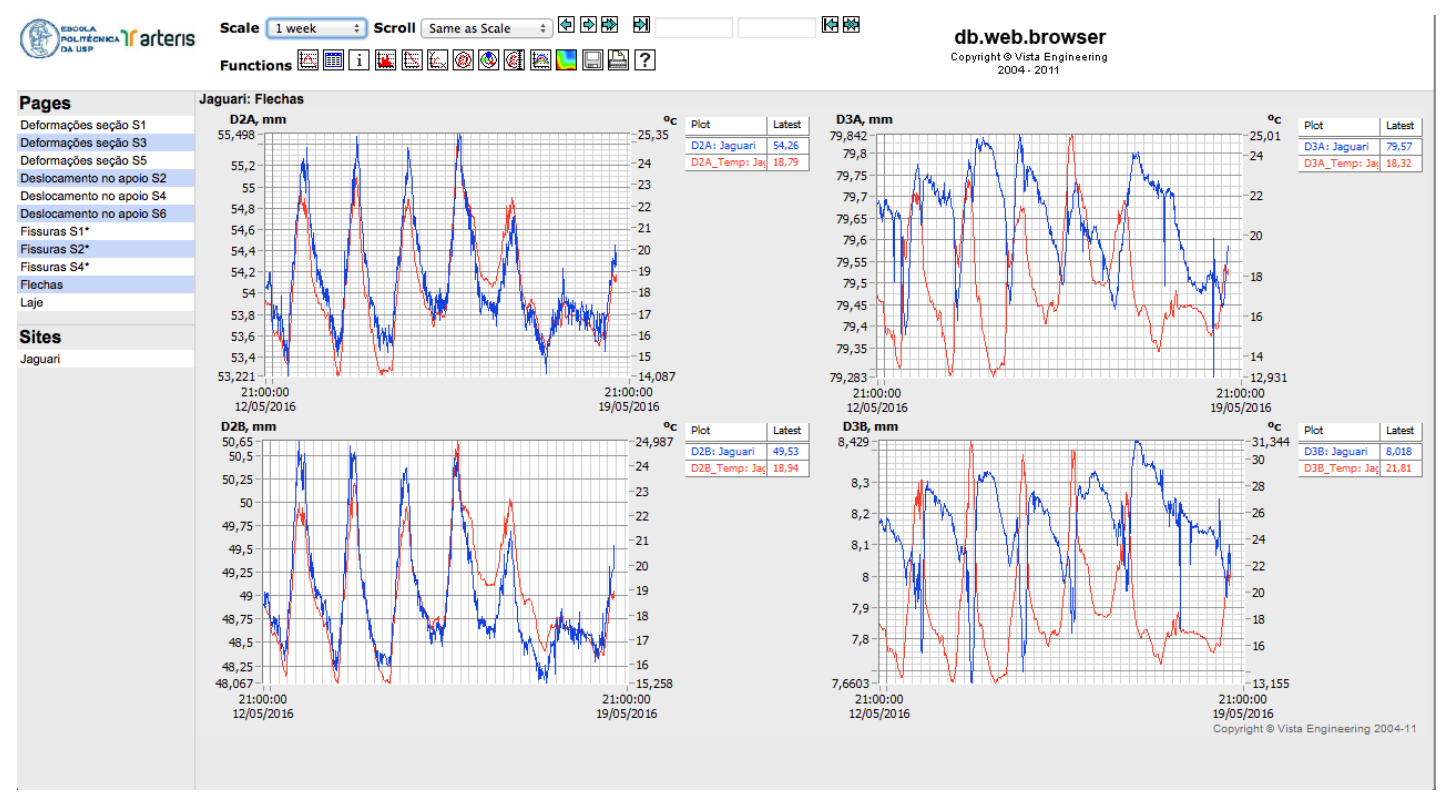

Figure 3.35.: Web interface for data visualization of the vibrating wire sensor data.

For live-load response data, however, the software package was not adequate and, therefore, a customized web visualization system was developed. Using the Java programming language routines for listing the collected data files, plotting the charts with the data for each event, and showing the collected images of the loading event were created. The resulting web page can be seen in Figure 3.36.

\subsection{Overview of the Collected Data}

This section presents a brief overview of the data collected during the long-term monitoring of the Jaguari River Bridge. It starts off by presenting the main findings from 
(1)

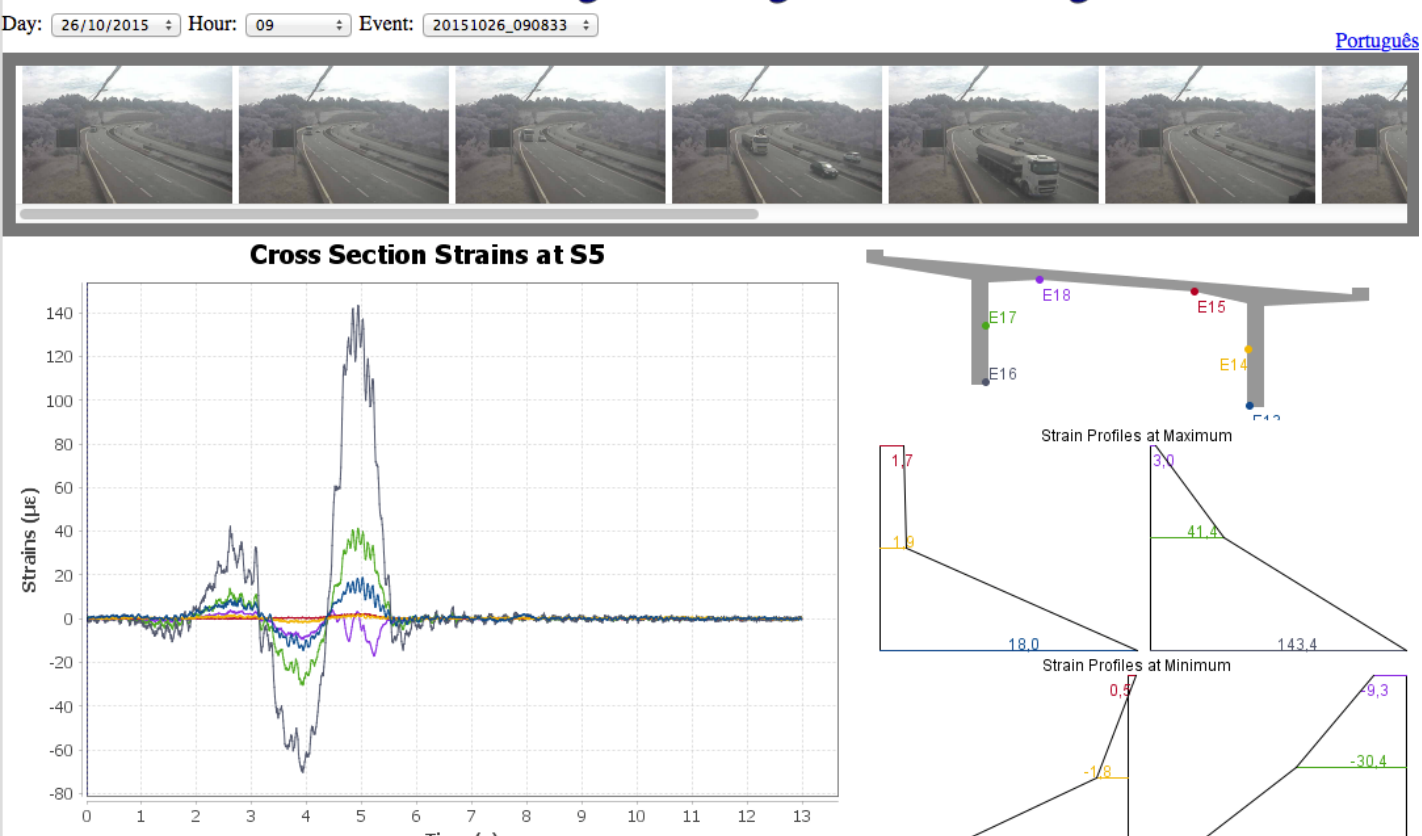

Figure 3.36.: Java based web interface for visualization of the recorded live-load data.

the live-load monitoring followed by a brief comparison between the data collected in the short and long-term monitoring. At last, the data collected by the vibrating wire sensors is analyzed. A complementary set of the data collected and processed in the structural testing, and short-term, and long-term monitoring of the Jaguari River Bridge is presented in Appendix A.

\subsubsection{Histograms of Live-Load Response}

As mentioned, data is stored by the long-term monitoring system whenever a significant loading event is registered. Using a MATLAB script, the maximum strains and displacements obtained for each of the registered events were determined. The data corresponding to the sensors installed at the bottom reinforcement of the longitudinal girders and deck slab are shown in the form of histograms in Figures 3.37 and 3.38.

Some similarities are clearly visible in the shapes of the histograms of the girder sensors. As expected, there is a clear difference between the shapes of the histograms for the sensors under the inner and outer girders. However, some slight changes are noticed in sensors E1 and E4 which are positioned at the larger of the five spans and due to the bridge geometry, larger lateral bending is expected at that point. 

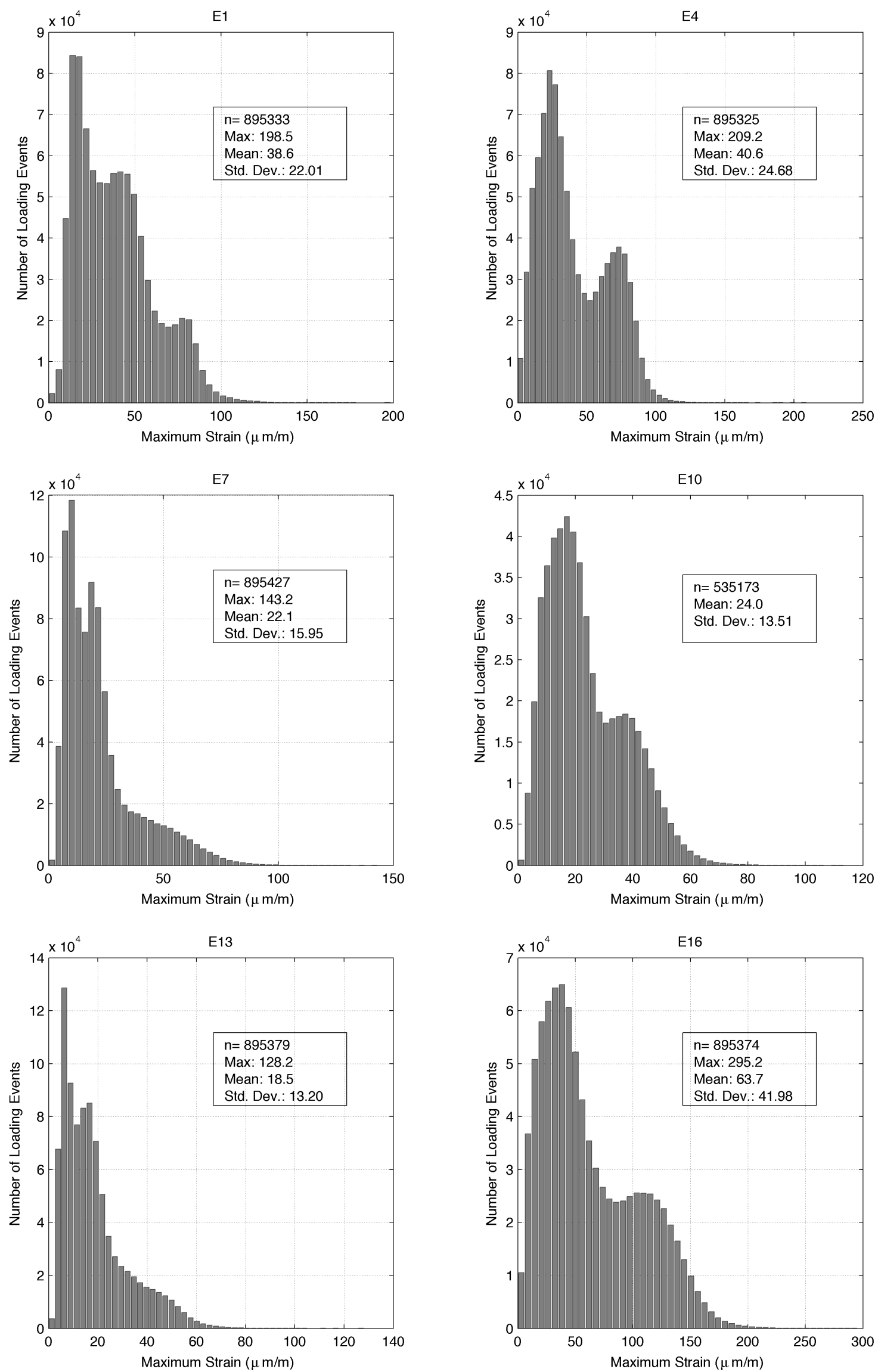

Figure 3.37.: Histograms of the maximum recorded strain for live-loading events in the bottom reinforcement of the girders. 
It is expected that the heavier loads tend to use the right lane of the bridge, in which case, the right wheel of the vehicles are almost over the outer girder. This loading condition sends most part of the load to the outer and a smaller part to the inner girder. The results show that there is a greater frequency of lighter loading acting in the inner girder which is, therefore, consistent. Further verification of this hypothesis is given by the histogram in Figure 3.38. Loading on the left lane tends to result in smaller strains at the middle of the slab, this was shown in the load testing that was carried out. The histogram for sensor E19 shows greater frequency for these smaller strains, suggesting more lighter loads at the left lane.

The measured strains are relatively low, which is expected for a reinforced concrete structure with these dimensions under service loads. During the processing of the data, the loading events that caused the largest strains were registered. The images from these loading events were then examined to determine the type of vehicle that crossed the bridge. For most of the sensors, the extreme values were due to the crossing of oversize load vehicles.

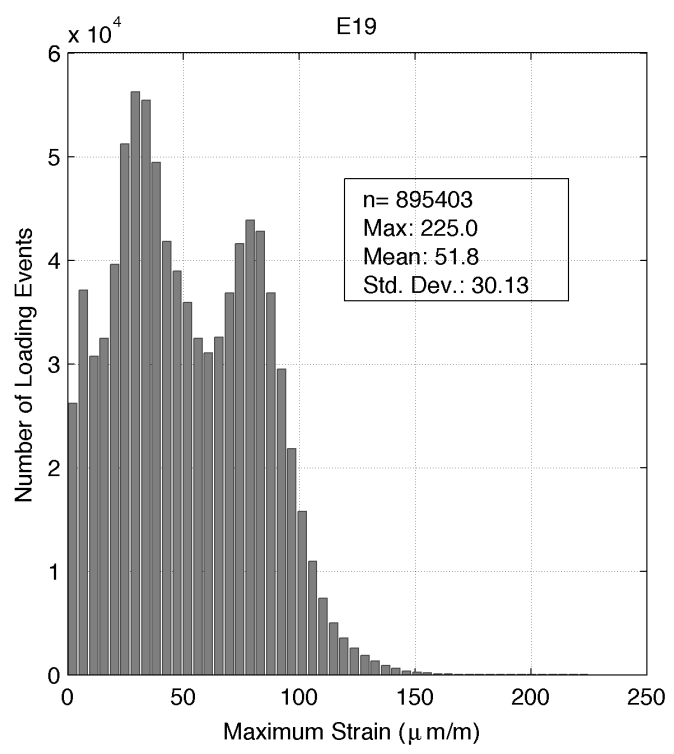

Figure 3.38.: Histograms of the maximum recorded strain for live-loading events in the bottom reinforcement of the deck slab.

\subsubsection{Comparison between Short-Term and Long-Term Monitoring}

In order to further verify the consistency of the collected data, comparison between the long-term and short-term monitoring was carried out. This comparison also allows for determining the influence of a longer monitoring period. In the figures below two sets of histograms are plotted, one for each case, also the data was fitted using kernel density functions which are plotted in the rightmost graph of the figures. 

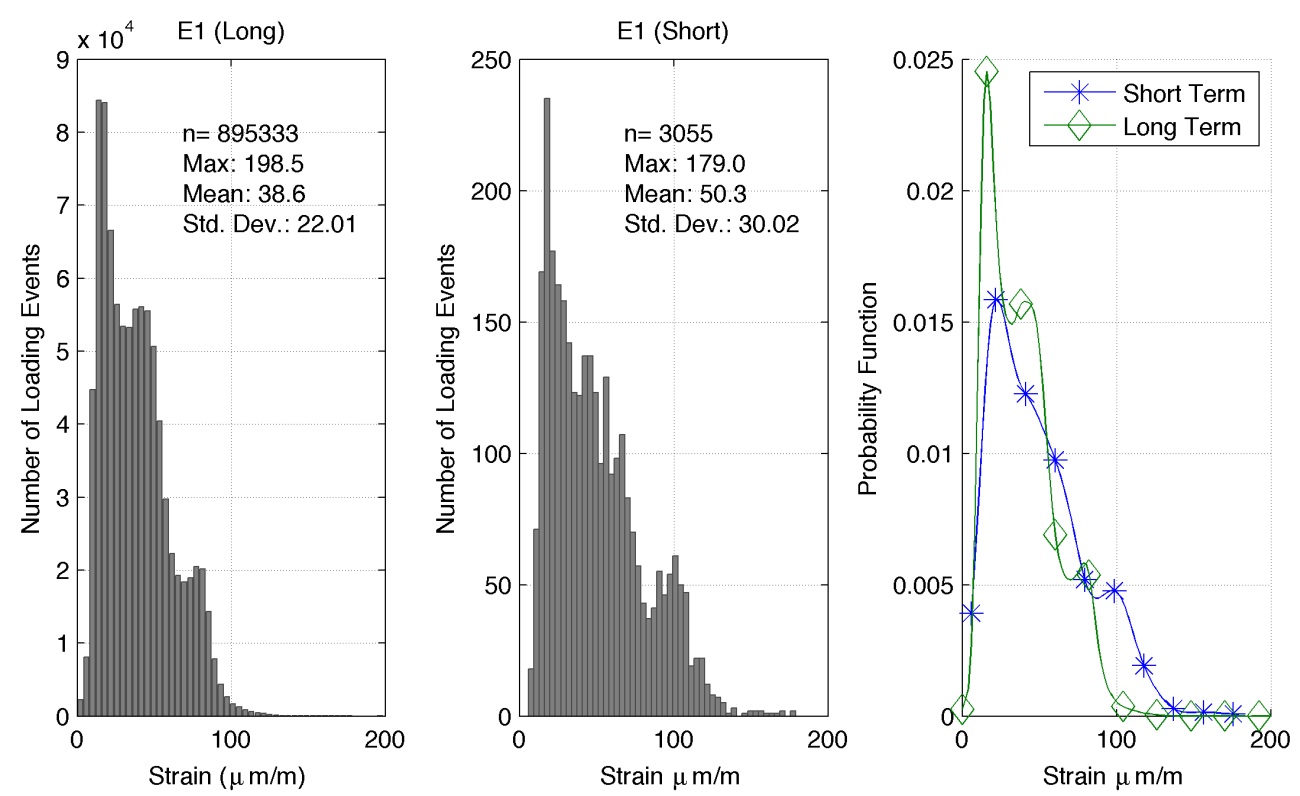

Figure 3.39.: Histograms and probability density plots comparing results for short and long-term monitoring of bottom reinforcement of inner girder in section S1.

Obviously the relatively small sample size obtained during the short-term monitoring allows for only limited conclusions. However, the considerable differences between the datasets shown in Figure 3.44 should be mentioned. In this case, significantly larger strain values were recorded during the long-term monitoring in comparison with the short-term data. Since the results of strain gauges is highly dependent on quality of the installation, an issue in that part may be the cause.

Overall, the data collected during both investigations seem to be consistent. Nevertheless, due to the relatively small sample size that was obtained during the short-term monitoring the response was not adequately determined. This can be seen in the comparisons between the kernel density functions, where these changes are significant specially at the upper tails. 

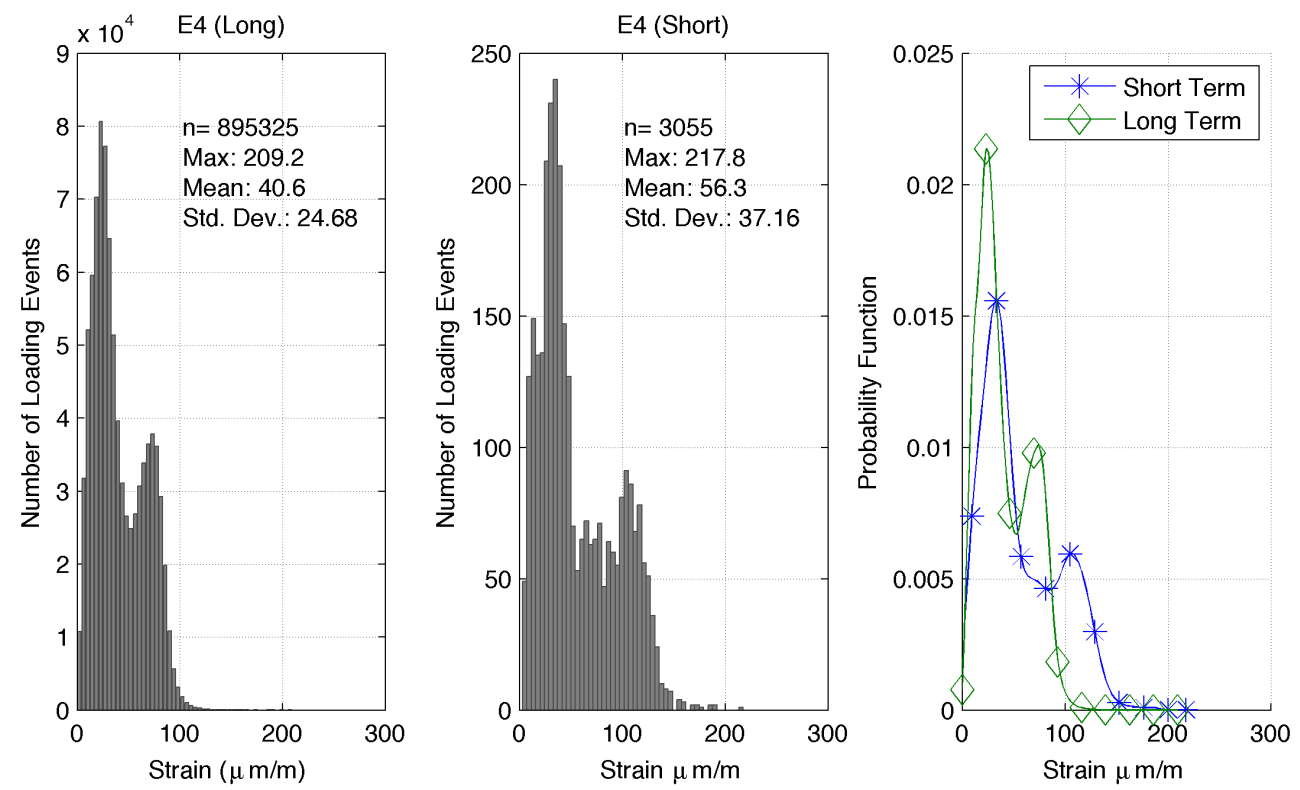

Figure 3.40.: Histograms and probability density plots comparing results for short and long-term monitoring of bottom reinforcement of outer girder in section S1.
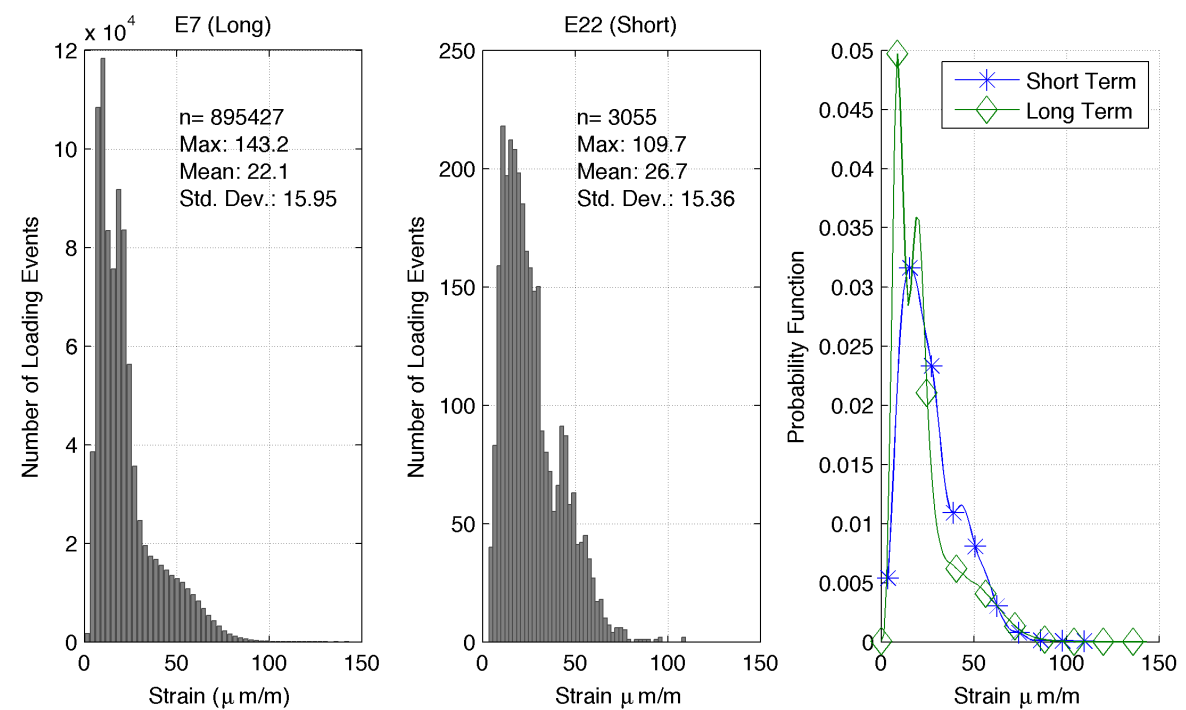

Figure 3.41.: Histograms and probability density plots comparing results for short and long-term monitoring of bottom reinforcement of inner girder in section S3. 

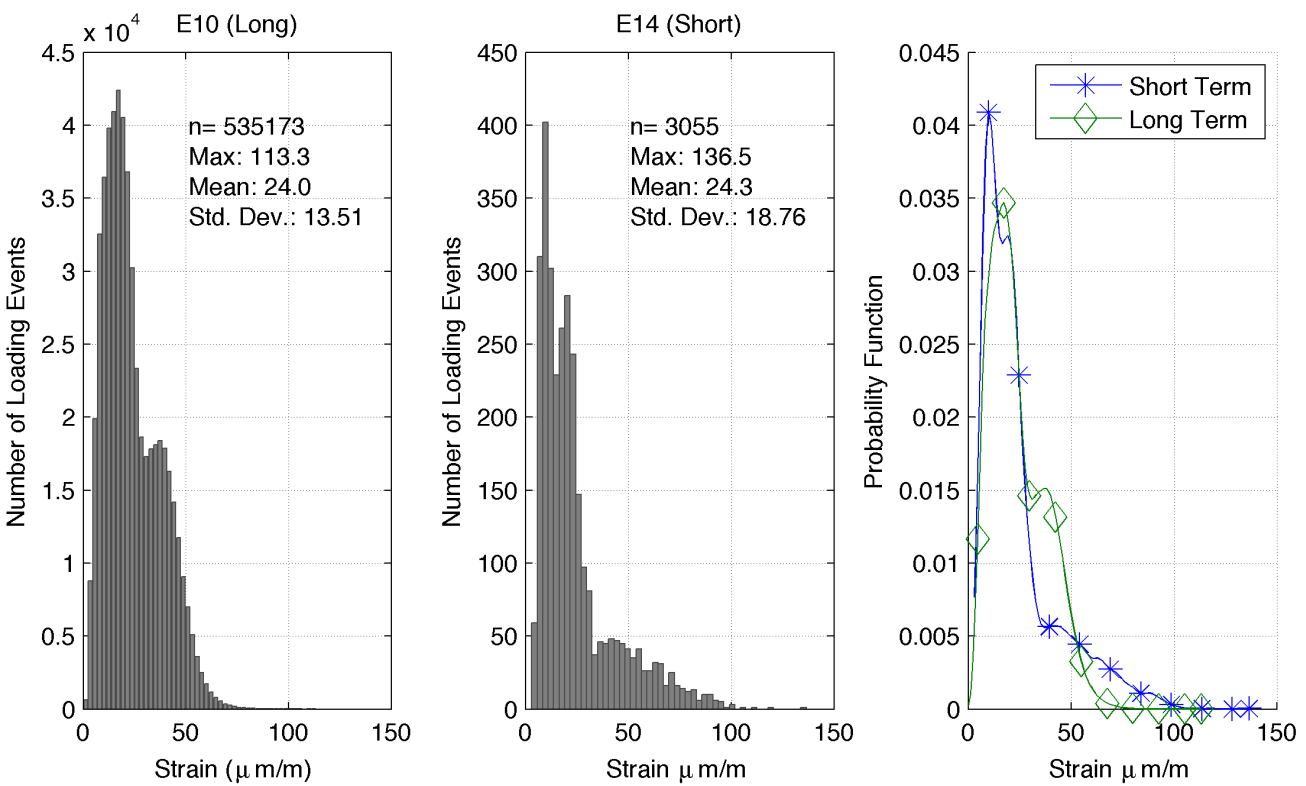

Figure 3.42.: Histograms and probability density plots comparing results for short and long-term monitoring of bottom reinforcement of outer girder in section S3.
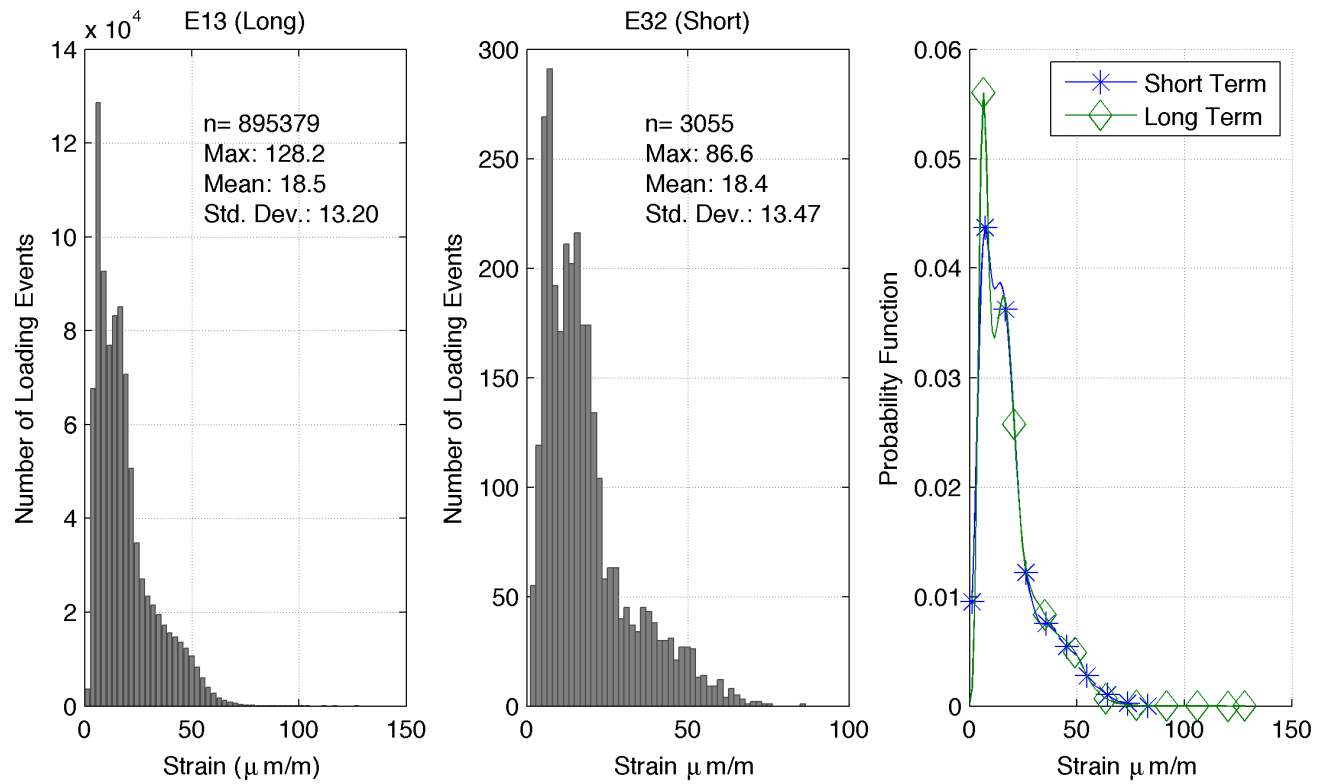

Figure 3.43.: Histograms and probability density plots comparing results for short and long-term monitoring of bottom reinforcement of inner girder in section S5. 

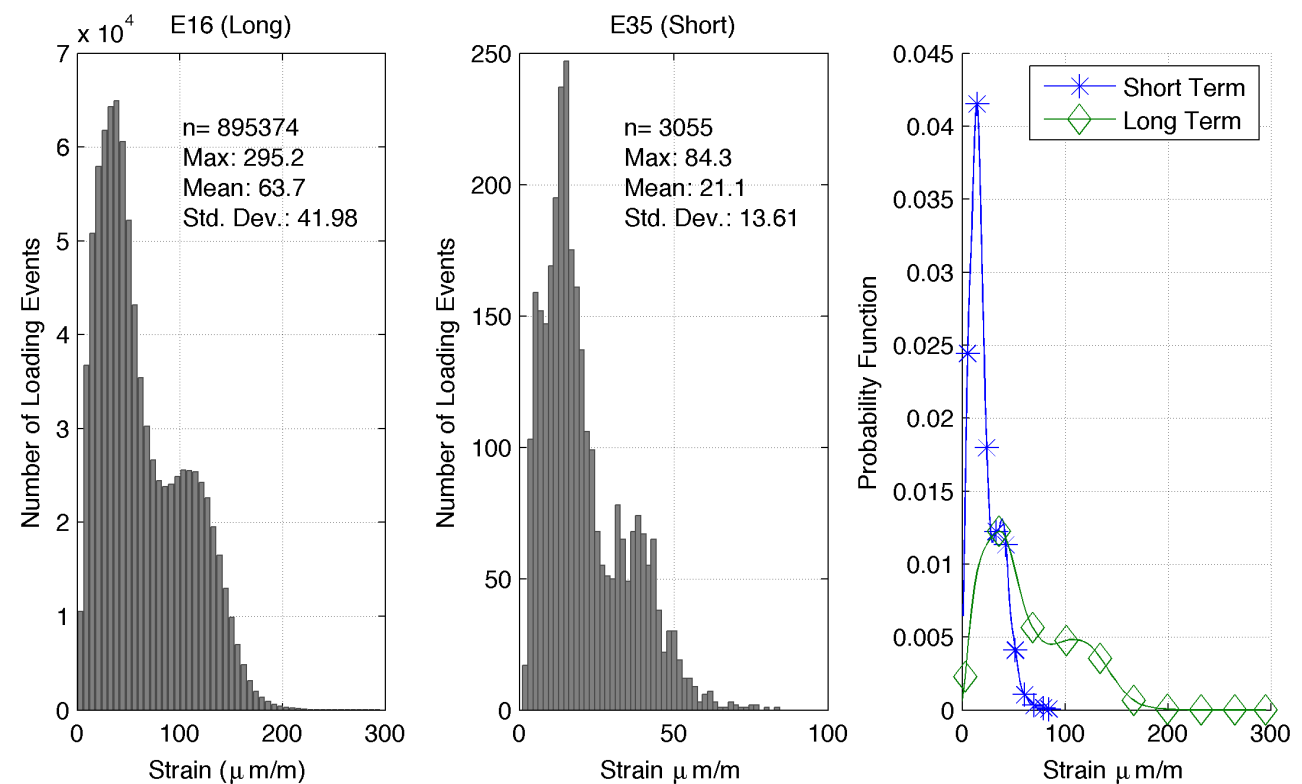

Figure 3.44.: Histograms and probability density plots comparing results for short and long-term monitoring of bottom reinforcement of outer girder in section S5.
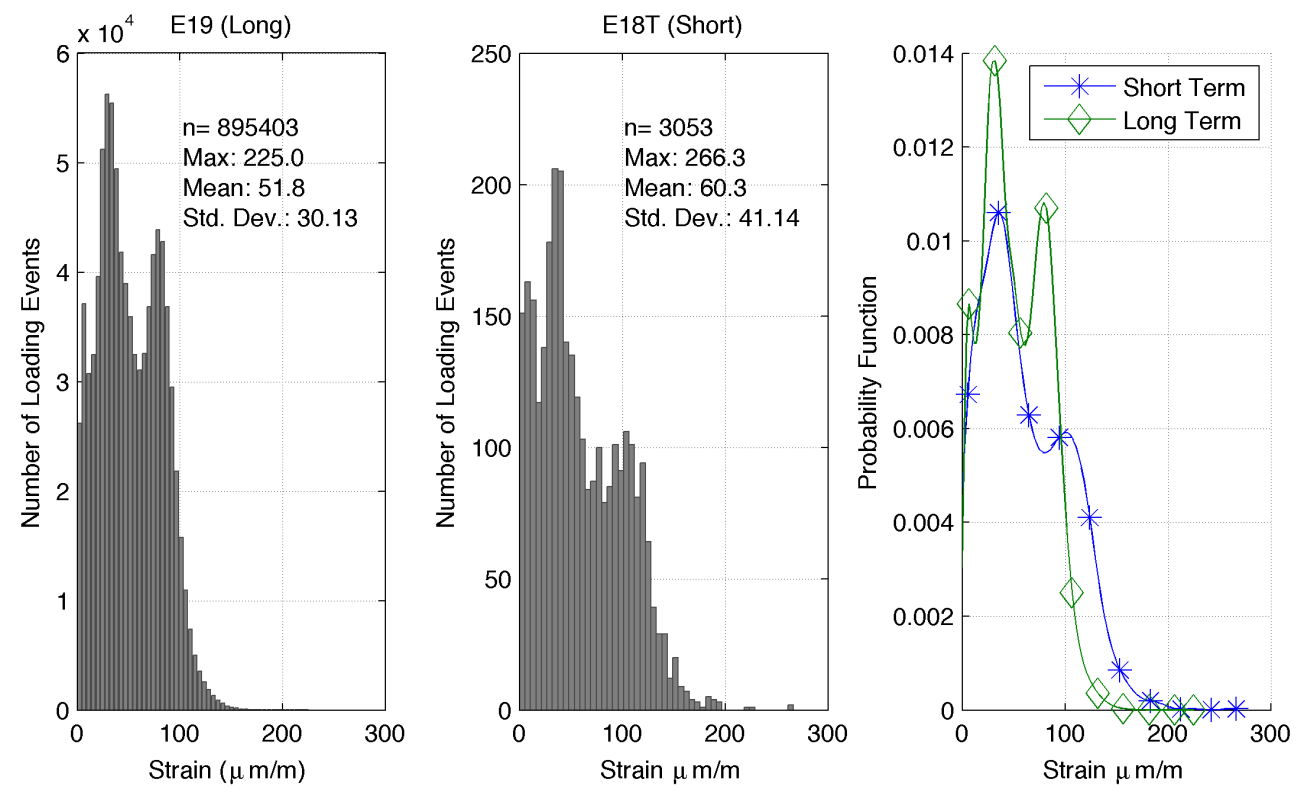

Figure 3.45.: Histograms and probability density plots comparing results for short and long-term monitoring of transverse bottom reinforcement of deck slab. 


\subsubsection{Vibrating Wire System}

In section A.3 the processed data from the vibrating wire sensors, collected between September of 2014 and July of 2015, is presented. The registered temperature variations were within the design limits which is a change of 10 to $15^{\circ} \mathrm{C}$ from the average temperature. No significant change in response, in the long term, was detected from this data. It can be observed that some signals show significant trends, however these follow the annual temperature variations.

\subsection{Final Remarks}

This chapter focused on the monitoring of the Jaguari River Bridge and the several stages of the implementation of this system. The installed monitoring system showed consistent results when compared to the short-term monitoring, as well as, to the results of numerical models. The monitoring system performed well and has been collecting data nonstop since September of 2014. Overall the data collected indicates that the structure is in good condition. 


\section{Probabilistic Life-Cycle Assessment Using Structural Health Monitoring}

\subsection{Introduction}

Data collected in SHM may have uncertainties associated with signal noise, calibrations errors, and/or installation issues. In this aspect, reliability theory concepts provide a reasonable approach for dealing with these uncertainties. This approach is also convenient because it allows for handling the uncertainties present in life-cycle assessment, as done in chapter 2. In this chapter, techniques for integrating the health monitoring data into reliability and life-cycle assessment will be addressed.

\subsubsection{General Overview of the Literature}

One of the earliest works with the objective of considering continuos monitoring for the reliability assessment of structures was presented by Rafiq [83]. In this paper a theoretical framework to update the corrosion initiation time based on embedded sensors in reinforced concrete bridges using a Bayesian technique is proposed. This framework was also applied in the development of bridge management strategies by the same author [82]. A reliability model for reinforced concrete bridge decks that incorporates probabilistic data from embedded corrosion rate sensors was presented by Marsh and Frangopol [55]. This model was also used in determining the optimal spatial distribution for these sensors [54].

Hosser, Klinzmann and Schnetgöke [36] proposed a framework for reliability based system assessment using SHM. The framework is based on probabilistic model that is used to determine the system failure probability and also a prognostic of the failure probability. This model also generates information regarding the random variables of the problem that can be used to determine which variables should be monitored and the frequency in which they should be monitored. Once the data collection begins, the model is updated using Bayesian methods allowing for adjustments on the monitoring plan. The proposed framework is applied, for demonstration, to a substitute structure which is subjected to the same stressors as a real bridge. 


\subsection{Introduction}

Frangopol, Strauss and Kim [32] demonstrate the use of monitoring data for the reliability assessment of a the Lehigh River Bridge SR-33. The monitoring program which was carried out consisted on measurements during the erection of the steel truss and the pouring of the concrete deck, park and moving-load testing and a long-term data collection of the temperature and live-load responses (January 2002 to March 2005)[20, 19]. The measured strains were transformed into stress using Hooke's law. A limit state of yielding of the truss elements was formulated by comparing the stresses due to dead loads and live loads to the yield strength of the material. All of the random variables in this analysis were characterized by their mean value and standard deviation and are assumed to be normally distributed. The reliability index of some of the truss elements are calculated during the construction, testing and service phases of the bridge; the effect of measurement errors is also investigated. A second performance indicator that was analyzed in this study is the fatigue behavior using the AASHTO Standard Specification for Highway Bridges [1]. Using the measured data, the effective stress ranges and number of cycles per day were determined for each sensor. The reliability analysis was carried out by comparing the allowed effective stress range with the measured stress range for each sensor. This type of reliability based assessment of fatigue life using SHM is a topic of special interest, due to the possibility of adequately characterizing the number of stress cycles and ranges through SHM while incorporating the measurement errors and model uncertainties in a probabilistic approach. This has been also explored by other authors([86, 67]).

Catbas, Susoy and Frangopol [14] presented an application to a long span truss bridge. Using 2D and 3D finite element models, which were calibrated through tests so that the behavior of the model would match the behavior of the actual structure, a reliability analysis of the bridge was undertaken. Dead, wind and vehicle loads were considered in a first analysis and the reliability indexes for each of the truss elements were determined using the First Order Reliability Method (FORM). By analyzing the long term strains that were recorded, the effect of temperature variations were characterized. These effects were fitted to a normal distribution and a new set of reliability indexes were determined for the truss elements. A system reliability analysis was also done using equivalent series/parallel systems. Significant differences were obtained between the member reliabilities and the system, indicating the importance of an accurate system modeling. The authors also mention that the temperature effects characterized through monitoring would have been very difficult to model using routine design analysis.

A general approach for performance prediction using monitored extreme data was proposed by Frangopol, Strauss and Kim [33]. Using polynomial prediction functions, a methodology is presented to determine the monitoring period and the prediction function coefficients in order to assure that the number of monitored extreme values that exceed the prediction function and the amount by which these value exceed the prediction function 
are limited. A time dependent reliability index associated with the difference between the prediction function and a given threshold is established. The probability of this reliability index falling below a certain limit (violation) is assumed to behave as Poisson process. With this approach, the time in between the occurrence of this violation can be used to determine monitoring interruption intervals. The use of Bayesian updating to determine the parameters of the extreme value considering the past monitored data is presented by Strauss, Frangopol and Kim [33] as an alternative to the use of the polynomial prediction functions.

Liu, Frangopol and Kim [48] proposed a safety assessment using a limit state equation based on measured strains and a strain limit. A random variable for the measurement error is inserted in equation to take this effect into consideration. In addition a condition function is considered for the case where the position of the sensor is not in a point of maximum strain. For predicting future maximum strain values, the maximum strain from each vehicle passage is fitted to a Gumbel distribution and the maximum expected strain in future years are obtained from this distribution. This methodology was applied to data from the Lehigh River Bridge SR-33; the strain limits were obtained from a proof load testing that was carried out, the data from different sensors were fitted to different distribution types. This limit state equation was also applied to the evaluation of bridge's system performance [49]. Sensitivity studies of the correlation of the sensor data, extreme value distribution type, measurement errors, and number of recorded vehicle passages were carried out in this study. Based on this study, the correlation was shown to have a significant effect on the system reliability; the measurement errors reduced the system reliability regardless of the system model; the type of distribution used did not influence the system reliability significantly due to the similar upper tails of the tested distributions; the number of observations reduced the uncertainty associated with the analysis; and the minimum number of observations may be defined by establishing a confidence interval.

Application of statistics of extremes to monitoring data for the reliability assessment and prediction of highway bridges was studied by Messervey, Frangopol and Casciati [58]. The authors used weigh-in-motion data from a 10-year study by [65] and SHM data from the Lehigh River Bridge SR-33 to study and develop a methodology for determining the extreme value distribution (EVD) using monitoring data. A methodology for obtaining the EVD for a timeframe from measured data and estimating the errors involved in this extrapolation is presented. These developments are also shown in Chapter 5 of [57], and the reader may also refer to this text for a good general overview of the possibilities regarding the use of SHM in assessment and management of civil infrastructure.

The use of Bayesian updating on the EVD using monitored live loads is presented in [73]. The updating is done based on a Metropolis-Hastings algorithm and a division of the data into smaller subsets is suggested in order to reduce numerical issues due to the likelihood 
function tending to zero as the sample size grows. In this paper, the use of SHM data in a reliability based life-cycle management framework is also demonstrated.

In the study by Orcesi and Frangopol [78], a performance indicator based on the cumulativetime failure probability (CTFP) due to heavy vehicle passage over a bridge was proposed. A methodology to calculate the indicator for each monitored component is presented. Using the indicators for each component, an importance index can be obtained and a methodology to detect potential damage or structural behavior changes is also proposed. The monitored data is also used to develop and test a framework to determine the optimal interruption intervals in the monitoring program. Other studies on optimization of the SHM systems can also be found in $[41,42,40]$.

\subsection{Live-Load Effect Characterization Using SHM Data}

As shown in the literature overview, data from SHM allows for the characterization of the actual live-load effects on the structure without having to recur to, sometimes complex, traffic simulations. In this section the characterization of these loading effects through the use of statistics of extremes is presented. The objective is to determine the live-load strain extreme value distribution (EVD) for return periods which can be used in the life-cycle assessment of a structure.

\subsubsection{Concepts of the Extreme Value Theory}

In engineering design and planning, it is fairly usual to have to deal with the occurrence of extreme events. Wether one is dealing with wind loads, river discharges, or traffic loads, the focus is associated, in most cases, with finding the probabilities associated with the occurrence of certain (extreme) values. The Extreme Value Theory provides a powerful toolset for dealing with problems in this context and, as mentioned above, was applied here for characterizing the extreme live loads. To provide a better context for understanding the data processing that was carried out for characterizing the extreme live loads through SHM, this subsection contains a brief overview of the main theoretical concepts of the Extreme Value Theory. For a more in-depth look the following references are recommended $[4,13,45,5]$.

To explain the basic concepts of the theory, consider the set $\left(X_{1}, X_{2}, \ldots, X_{n}\right)$ made up of $n$ observations of a given population. The random variables associated with minimum and maximum values of this set are defined as $Y_{1}=\min \left(X_{1}, X_{2}, \ldots, X_{n}\right)$ and $Y_{n}=\max \left(X_{1}, X_{2}, \ldots, X_{n}\right)$. It can be shown that if $\left(X_{1}, X_{2}, \ldots, X_{n}\right)$ is made up of statistically independent samples that are identically distributed according to $X$, then 
the cumulative distribution function $(\mathrm{CDF})$ and probability distribution function (PDF) of the minimum, $Y_{1}$, and maximum, $Y_{n}$, can be defined as below.

The CDF and PDF of the distribution of the minimum $\left(Y_{1}\right)$ for the set are given, respectively, as:

$$
\begin{aligned}
& F_{Y_{1}}(y)=1-\left[1-F_{X}(y)\right]^{n} \\
& f_{Y_{1}}(y)=n\left[1-F_{X}(y)\right]^{n-1} f_{X}(y)
\end{aligned}
$$

For the maximum $\left(Y_{n}\right)$ of the set, the CDF and PDF are respectively:

$$
\begin{aligned}
& F_{Y_{n}}(y)=F_{X}(y)^{n} \\
& f_{Y n}(y)=n\left[F_{X}(y)\right]^{n-1} f_{X}(y)
\end{aligned}
$$

The equations above determine the exact distribution of the extreme values of a dataset of $n$ independent occurrences of $X$. It can be shown $[13,45]$ that, the above CDF's degenerate as $n$ tends to infinity. In other words, as $n$ tends to infinity the result of the CDF's will either be 0 or 1 . To overcome this problem, linear transformations are used and a set of limiting no-degenerate distributions are obtained. This process results in three families of asymptotic EVD's: Type I - Gumbel, Type II - Frechet, and Type III Weibull, depending on the tail behavior of the distribution of $X$.

\subsubsection{Type I - Gumbel Distribution for Live-Load Data}

In this chapter, the Type I - Gumbel EVD will be used to characterize the maximum values of live-load data. The choice of this distribution was based on the fact that it has been shown to adequately model the maximum loads of WIM data [70]. In order to formalize the basic concepts that are applied in the next sections, the basic equations regarding this distribution are shown here. The CDF and PDF for the Gumbel distribution 
for maxima are:

$$
\begin{aligned}
& F_{Y_{n}}(y)=\exp \left[-e^{-\alpha_{n}\left(y-u_{n}\right)}\right] \\
& f_{Y_{n}}(y)=\alpha_{n} e^{-\alpha_{n}\left(y-u_{n}\right)} \exp \left[-e^{-\alpha_{n}\left(y-u_{n}\right)}\right]
\end{aligned}
$$

In these equations, $u_{n}$ is the most probable value of $Y_{n}$ (location parameter) and $\alpha_{n}$ is the shape factor (shape parameter). These parameters can be related to the mean $\left(\mu_{Y_{n}}\right)$ and variance $\left(\sigma_{Y_{n}}^{2}\right)$ of $Y_{n}$ according to:

$$
\begin{aligned}
& \mu_{Y_{n}}=u_{n}+\frac{\gamma}{\alpha_{n}} \\
& \sigma_{Y_{n}}^{2}=\frac{\pi^{2}}{6 \alpha_{n}^{2}}
\end{aligned}
$$

Where $\gamma \approx 0.5772$ is the Euler-Mascheroni constant. By definition, $u_{n}$ is the value that, in a set of $n$ values of $X$, only one value is expected to be larger than $u_{n}$, therefore:

$$
n\left[1-F_{X}\left(u_{n}\right)\right]=1
$$

which, rearranged is:

$$
F_{X}\left(u_{n}\right)=1-\frac{1}{n}
$$

Therefore, with the CDF of the parent distribution, $F_{X}(x)$, known, $u_{n}$ may be obtained using eq. 4.9. Therefore, by using eq. 4.3 to calculate some points of $F_{Y_{n}}(y)$ to obtain $\alpha_{n}$ through a curve fitting method, both distribution parameters may be determined.

Another characteristic of this distribution that needs to be mentioned, regards the case where the random variable $X$ follows a Gumbel distribution. For this specific case, when eq. 4.3 is applied to eq. 4.5 , one obtains the relationships shown in eq. 4.10 , where $u_{X}$ 
and $\alpha_{X}$ are the parameters for the Gumbel distribution of $X$.

$$
\begin{gathered}
\alpha_{n}=\alpha_{X} \\
u_{n}=u_{X}+\frac{\ln (n)}{\alpha_{n}}
\end{gathered}
$$

Essentially this result demonstrates that, for this specific case, the shape factor of the two distributions stay the same, while the location parameter suffers a shift dependent on $n$. As shown in another part of this chapter, this characteristic can be used for obtaining estimates of the EVD for larger timeframes (1, 10 or 50-year) based on smaller ones (1, 3, 7-day).

\subsubsection{Application to the Jaguari River SHM Data}

Having established the basic concepts associated with the Extreme Value Theory, it is now possible to move on to the applications of this toolset to data obtained at the Jaguari River monitoring site. The main objective here is to obtain the distribution for the maximum yearly live-load strain that will be used in the life-cycle analysis in section 4.3. Two strategies are applied for this purpose: one assumes a Gumbel distribution for the extreme data in shorter timeframes (1-day, 3-days, etc.) and then uses the equations derived in section 4.2.1 to transform to the 1-year timeframe, while the other uses kernel density estimates to determine the parent distribution of the live-load strain before transforming it to the appropriate timeframe. For brevity, in the analysis that were carried out throughout this chapter, only data from strain gauge E16 was used.

Since these transformation methods rely on the assumption that the collected data is part of a population which follows a certain probability distribution, homogeneity of the data is important for attaining consistent results. Therefore, before applying these methods, a general evaluation of homogeneity of the data was carried out.

\subsubsection{Homogeneity of the Collected Live-Load Data}

The collected live-load data, as mentioned in Chapter 3, consists on a series of strain time-histories that correspond to loading events. As shown in the sections that follow, the data was compiled into sets of maximum strains per loading event and maximum daily, weekly and other timeframes.

Since the collected strain data is highly dependent on the traffic loading pattern which, may be subject to seasonal fluctuations. Any heterogeneity that yield from these fluctuations have to be removed in order to obtain consistent results. In section 4.2.2.3, an 
extrapolation is carried out by transforming the maximum strain per loading event distribution into a EVD for a 1-year timeframe. In order to do this, the number of daily loading events is used. Therefore, the homogeneity verification will start by examining if there are any significant seasonal changes in the average number of daily loading events.

The 895,442 loading events, that were recorded during 378 days between September 2014 and October 2015 at the Jaguari River Bridge site, were sorted by month and day of the week in which the event occurred. Figure 4.1 presents the results of this process in terms of mean and standard deviations of the daily number of loading events. It can be seen that the daily number of loading events during the weekdays (Monday to Friday) is practically constant while, during the weekends, this value is significantly lower. Also, although there are some changes during the different months of the year, no trend is observed. These results suggest that, for the transformation in section 4.2.2.3, more consistent results would be obtained by using only the data collected during the weekdays.

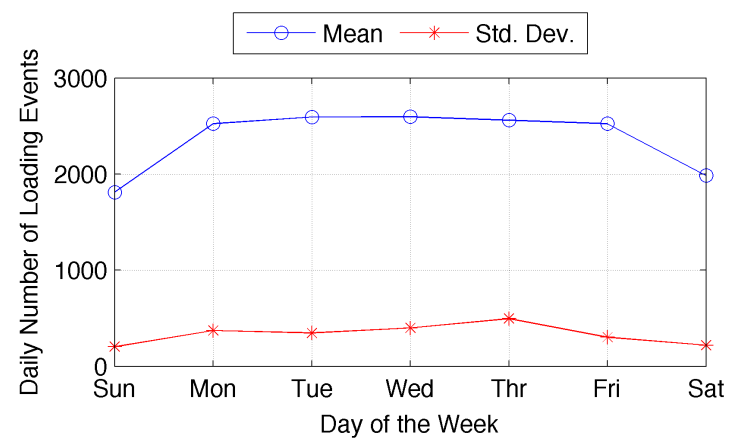

(a)

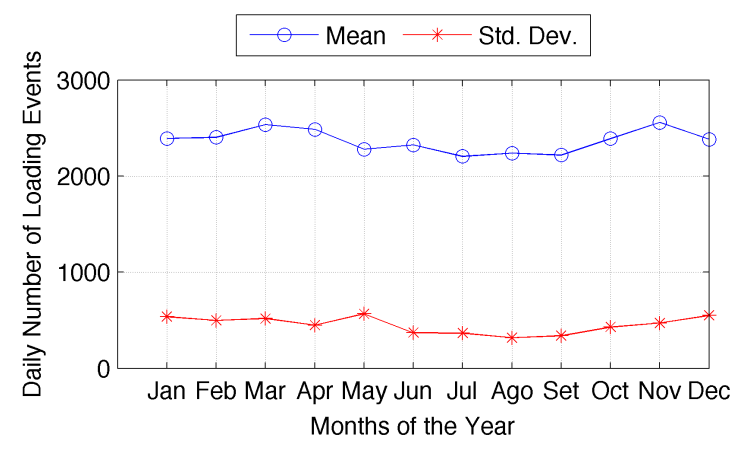

(b)

Figure 4.1.: Mean and standard deviation of the number of daily loading events sorted by: (a) day of the week; and (b) month of the year.

One other homogeneity test was carried out regarding the maximum strain values recorded during each loading event. For this purpose, the 895,442 values of the data vector were also separated per day of occurrence and the mean and standard deviation were calculated for each day of monitoring. These parameters serve as indicators of the behavior of the maximum strain per loading event throughout the different days of monitoring. This data was then separated by month and day of the week of occurrence. The finding of this compilation are presented in Figure 4.2. It shows that the mean maximum strain for the loading events are generally stable during the weekdays and show deviations during the weekends. This result is consistent with the findings on the average number of loading events per day. Although some fluctuations, of these parameters, are found throughout the different months of the year, no significant seasonal effects can be observed.

Regarding the recorded daily maximum values, its consistency may also be checked by sorting these values in terms of day of the week and month of the year. In the same 


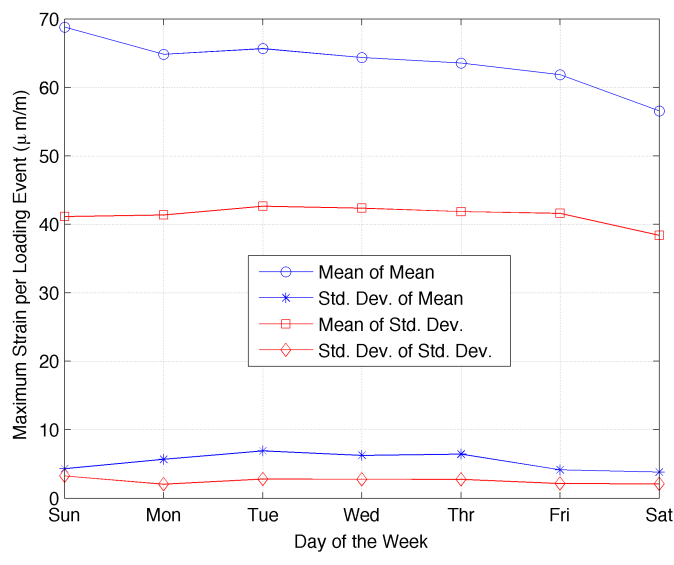

(a)

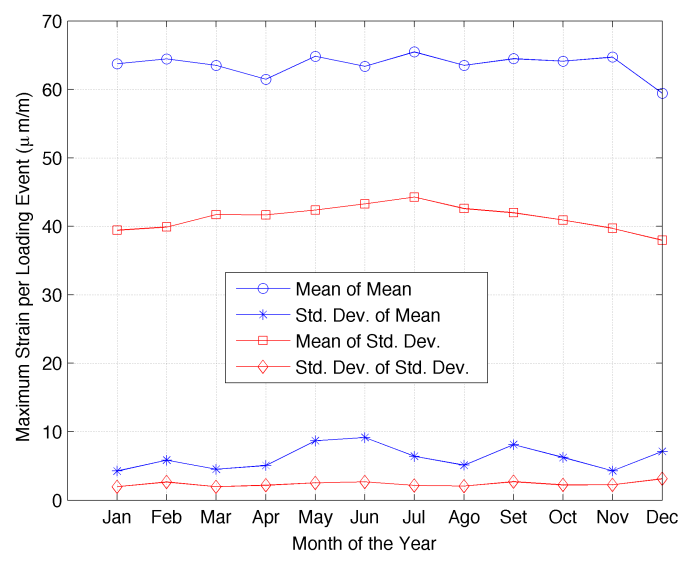

(b)

Figure 4.2.: Plots showing the behavior of the mean and standard deviation of the means and standard deviations of the maximum strain per loading event relative to: (a) day of the week and; (b) month of the year.

manner as above, the sorted daily maximum data, presented in Figure 4.3, is shown in terms of mean and standard deviation. Additionally, the maximum value obtained for all weeks and months of monitoring are shown as well. Although there are a few drops in the maximum recorded values (take into consideration the month of August for example), the mean values and standard deviations do not show significant changes throughout the different days of the week and months of the year. These results indicate that the maximum daily strain is not influenced by seasonal effects.

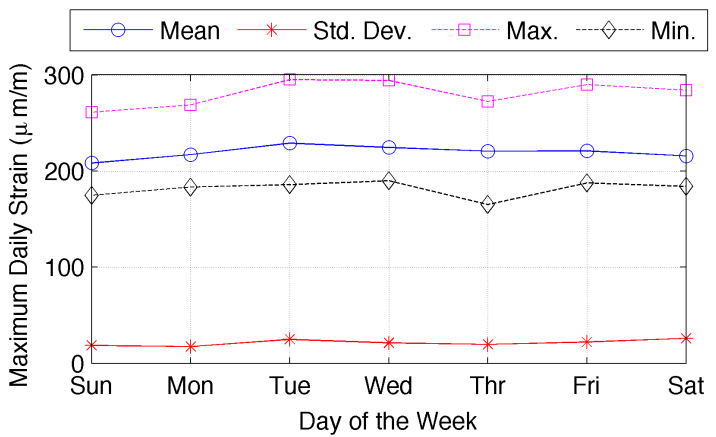

(a)

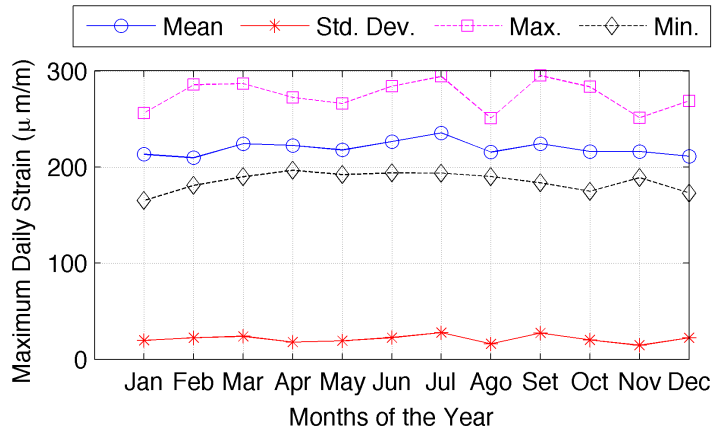

(b)

Figure 4.3.: Mean and standard deviation of the number of daily loading events sorted by: (a) day of the week; and (b) month of the year.

With the homogeneity of the data checked, the different strategies for determining the distribution of the maximum yearly live-load strain can be applied to the data. Since the daily maximum strain there are no apparent restrictions on using all of the collected data. However, with regard to the maximum strain per loading event, the analysis should consider only the data collected during the weekdays. The following sections show the 
results of the different strategies.

\subsubsection{Transformation assuming Gumbel Distribution}

By processing the collected data and obtaining the maximum recorded strains in $1,3,7$, 14, and 30 day intervals and fitting Gumbel distributions to this data, using maximum likelihood estimation (MLE) [5], the respective distributions for these timeframes are determined. Through eq. 4.10 it is possible to estimate the Gumbel distribution parameters associated with a year long timeframe, i.e., distribution of yearly maximum strain. This strategy was based on the methodology presented by Messervey et. al. [57, 58].

\begin{tabular}{c||c|c|c|c|c} 
Timeframe & 1-day & 3-day & 7-day & 14-day & 30-day \\
\hline \hline Mean $\left(\mu_{X}\right)$ & 219.8 & 238.9 & 253.7 & 263.3 & 277.5 \\
Std. Dev. $\left(\sigma_{X}\right)$ & 23.6 & 22.8 & 24.0 & 18.4 & 16.8 \\
Sample Size & 378 & 126 & 54 & 27 & 12 \\
$n$ & 365 & 121.7 & 52.1 & 26.1 & 12.2 \\
Mean yearly $\left(\mu_{Y_{\text {1year }}}\right)$ & 328.2 & 324.3 & 327.7 & 310.0 & 310.1 \\
Std. Dev. yearly $\left(\sigma_{Y_{\text {1year }}}\right)$ & 23.6 & 22.8 & 24.0 & 18.4 & 16.8
\end{tabular}

Table 4.1.: Results of the MLE of Gumbel distribution for the 1, 3, 7, 14, and 30-day maximum strain, along with the, respective, transformation to the yearly maximum strain distribution.

Table 4.1 summarizes the results of the data processing along with the transformation for the one year timeframe. The obtained results show two different trends, one presents the yearly maximum distribution with mean around $326 \mu \mathrm{m} / \mathrm{m}$, for the 1,3 , and 7 -day timeframes, while for the 14, and 30-day timeframes this mean is around $310 \mu \mathrm{m} / \mathrm{m}$. As shown in Figure 4.4 there are two sets of resulting distributions which present significant differences. The different results obtained in the transformations are due to the differences in the standard deviations obtained in each set of data $(1,3,7,14$, and 30-day).

As mentioned in section 4.2.2.1, the homogeneity of the collected data is important in the distribution characterization process. When working with short timeframes, for instance one or three days, changes in the traffic characteristics due to decrease in commercial trucking during weekends and holidays may generate heterogeneities in the collected data. Therefore, by using a larger observation timeframe, it is possible to have less variability in the collected data which leads to a better suited distribution for transforming to larger timeframes. In another study, which used the monitored live-load effect data from the Lehigh River Bridge SR-33, the use of 3-day observation timeframe yielded lower standard deviations than obtained by 1, 2, 6, 9, and 12-day observation timeframes [58]. This study was, however, carried out using data from only 90 days of monitoring which leaves a limited amount of samples for characterizing the larger timeframes. 


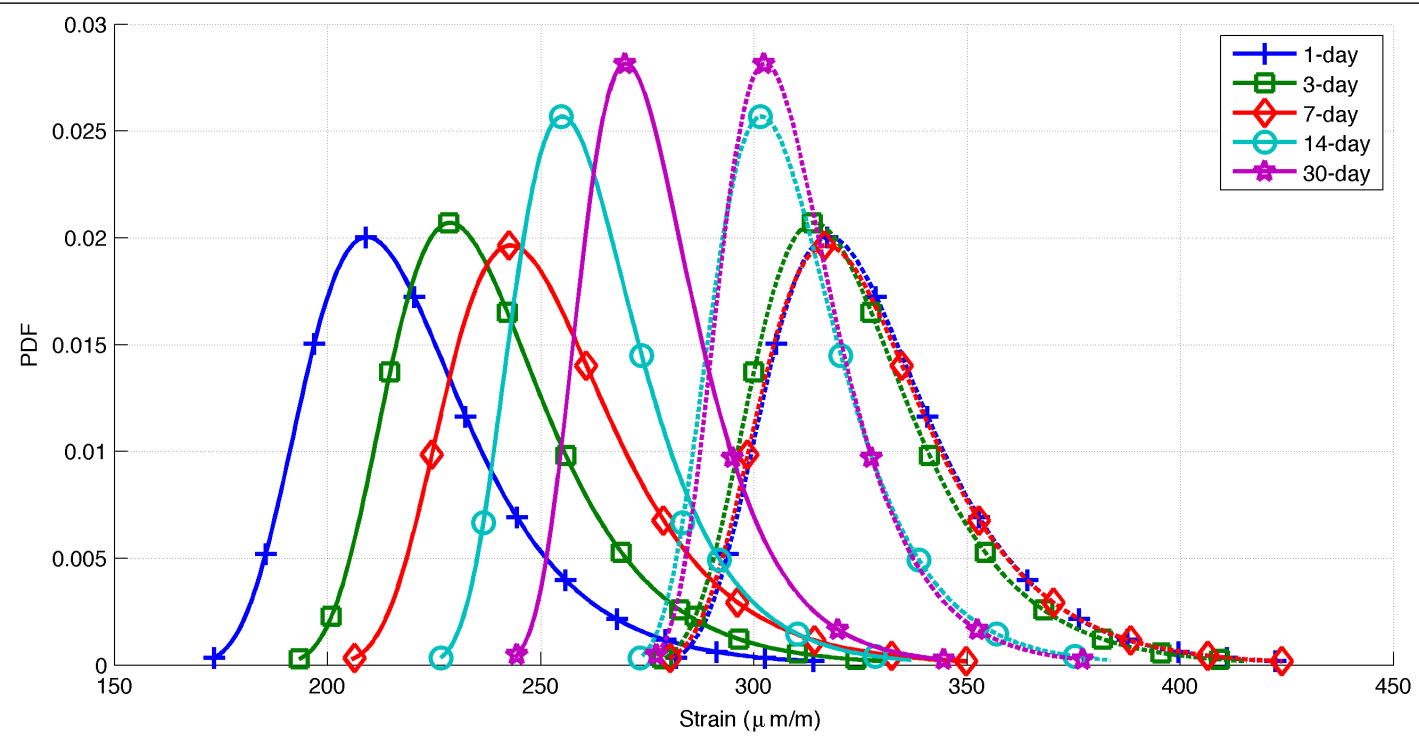

Figure 4.4.: Probability density plots (solid lines) of the Gumbel MLE, for the different timeframes considered, and, respective, shifted yearly maximum distribution (dashed lines).

Obviously, the better data homogeneity associated with a larger observation timeframe comes with the price of a longer monitoring period in order to be able to adequately characterize it. Hence, the objective in this case is to determine a timeframe which results in a low standard deviation that has an adequate number of samples. By tracking the rolling average and standard deviation of the data samples (see Figure 4.6), it is possible to check if convergence of these parameters is obtained. The fact that these parameters stabilize once the samples reach a certain size is an indicator that the there are sufficient samples have been considered. It should be mentioned, however, that although this stabilization indicates that the sample size is adequate it is not a guarantee that this sample properly characterizes the population.

From the results of the rolling mean and standard deviation shown in Figure 4.6, of the collected data for the different timeframes considered, it is unclear wether the available datasets are adequate for characterizing the associated distribution parameters. There is some indication of stabilization for the daily maximum data, however for the other timeframes there is still some fluctuation. These result do not allow for asserting which transformation from Table 4.1 is better suited for use.

In this case, another option would be to try and censor the data in order to remove any heterogeneities. Based on the results from the homogeneity study of the data from the Jaguari River Bridge, the data from the weekends was removed. Additionally days that fell on public holidays were also removed from the analysis. Using MLE to determine the Gumbel fits for this censored data the results shown in Table 4.2 and Figure 4.5 were obtained. From these results, it can be observed the standard deviations associated 
with the considered timeframes suffered slight changes which, in turn, yielded much more consistent transformed yearly maximum distributions when compared to the ones obtained from the uncensored data.

Although the censoring of data reduces the total number of available datasets (at least for some of the shorter timeframes), as shown in Figure 4.7, the rolling mean and standard deviation seem to stabilize with a fewer number of samples than when compared to the uncensored data. This is expected since heterogeneities slow down the convergence process by the presence of data points that are unrepresentative of the underlying population.

\begin{tabular}{c||c|c|c|c|c} 
Timeframe & 1-day & 3-day & 5-day & 10-day & 20-day \\
\hline \hline Mean $\left(\mu_{X}\right)$ & 223.1 & 240.8 & 250.0 & 261.3 & 272.9 \\
Std. Dev. $\left(\sigma_{X}\right)$ & 21.6 & 21.9 & 20.7 & 19.4 & 18.8 \\
Sample Size & 260 & 86 & 52 & 26 & 12 \\
$n$ & 254 & 84.7 & 50.8 & 25.4 & 12.7 \\
Mean yearly $\left(\mu_{Y_{\text {1year }}}\right)$ & 316.3 & 316.6 & 313.6 & 310.3 & 309.6 \\
Std. Dev. yearly $\left(\sigma_{Y_{\text {1year }}}\right)$ & 21.6 & 21.9 & 20.7 & 19.4 & 18.8
\end{tabular}

Table 4.2.: Results of the MLE of Gumbel distribution for the 1, 3, 5, 10, and 20-day maximum strain for business days, along with the, respective, transformation to the yearly maximum strain distribution.

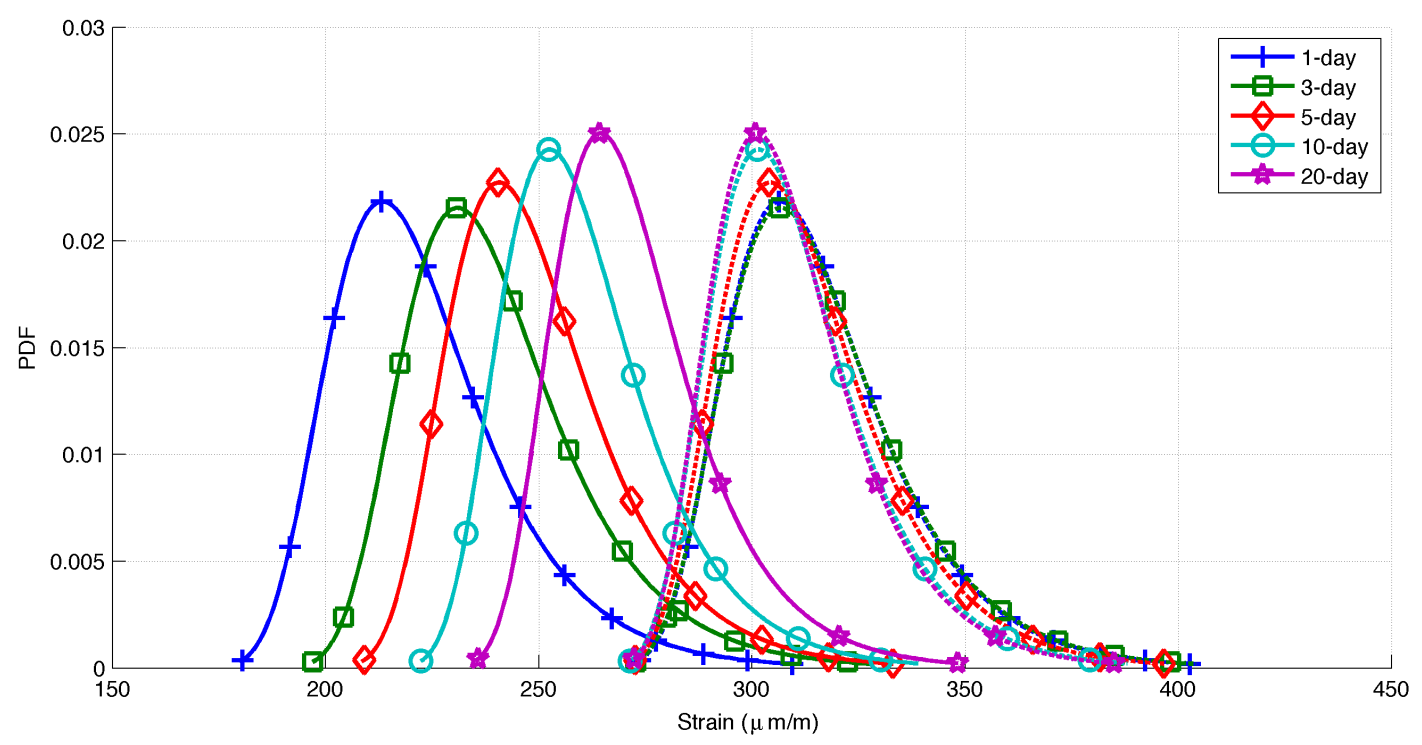

Figure 4.5.: Probability density plots (solid lines) of the Gumbel MLE for the censored data (only business days), for the different timeframes considered, and, respective, shifted yearly maximum distribution (dashed lines). 

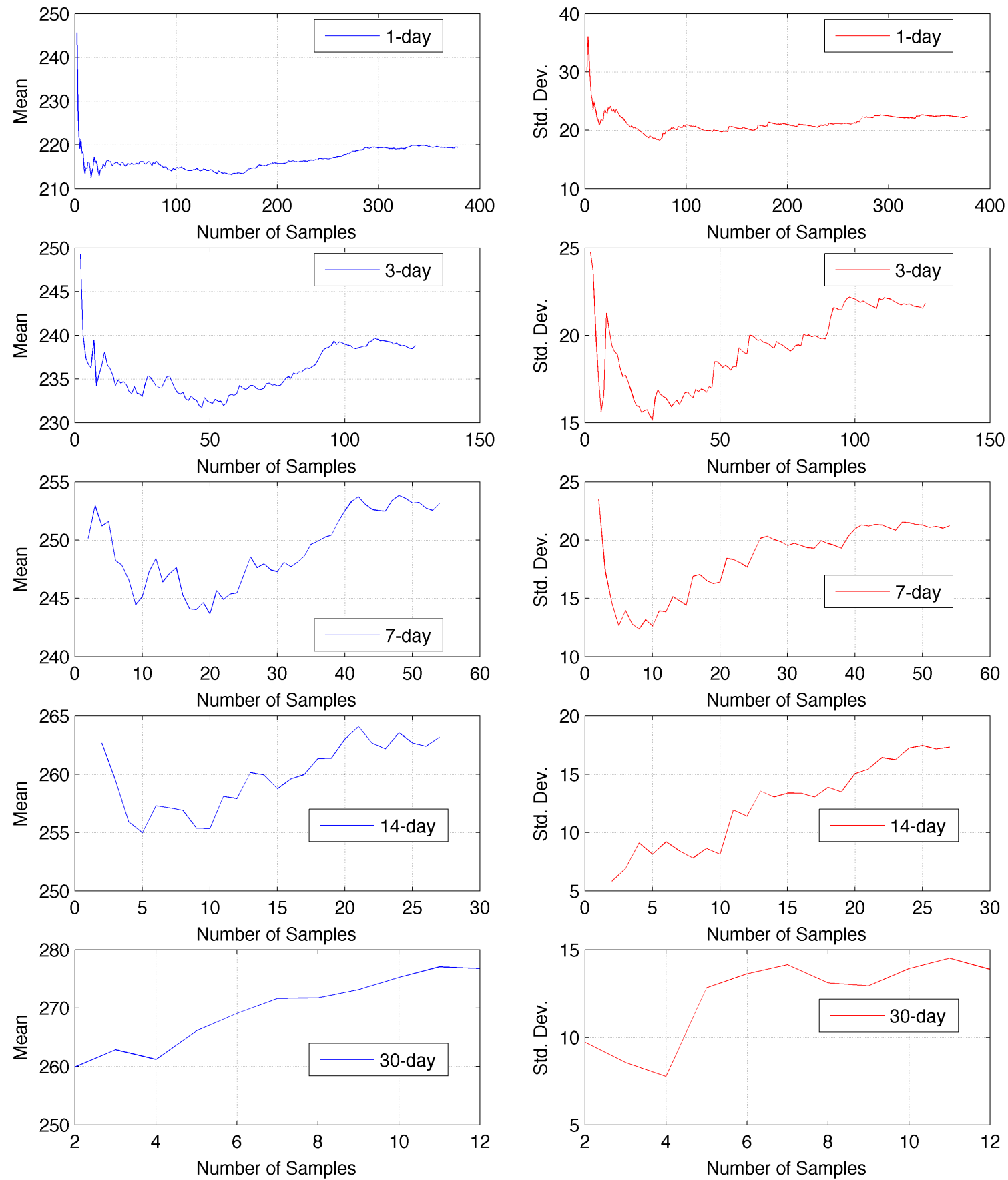

Figure 4.6.: Rolling mean and standard deviation of the collected data samples; in these plots, the parameters are calculated for an increasing number of samples to check convergence. 

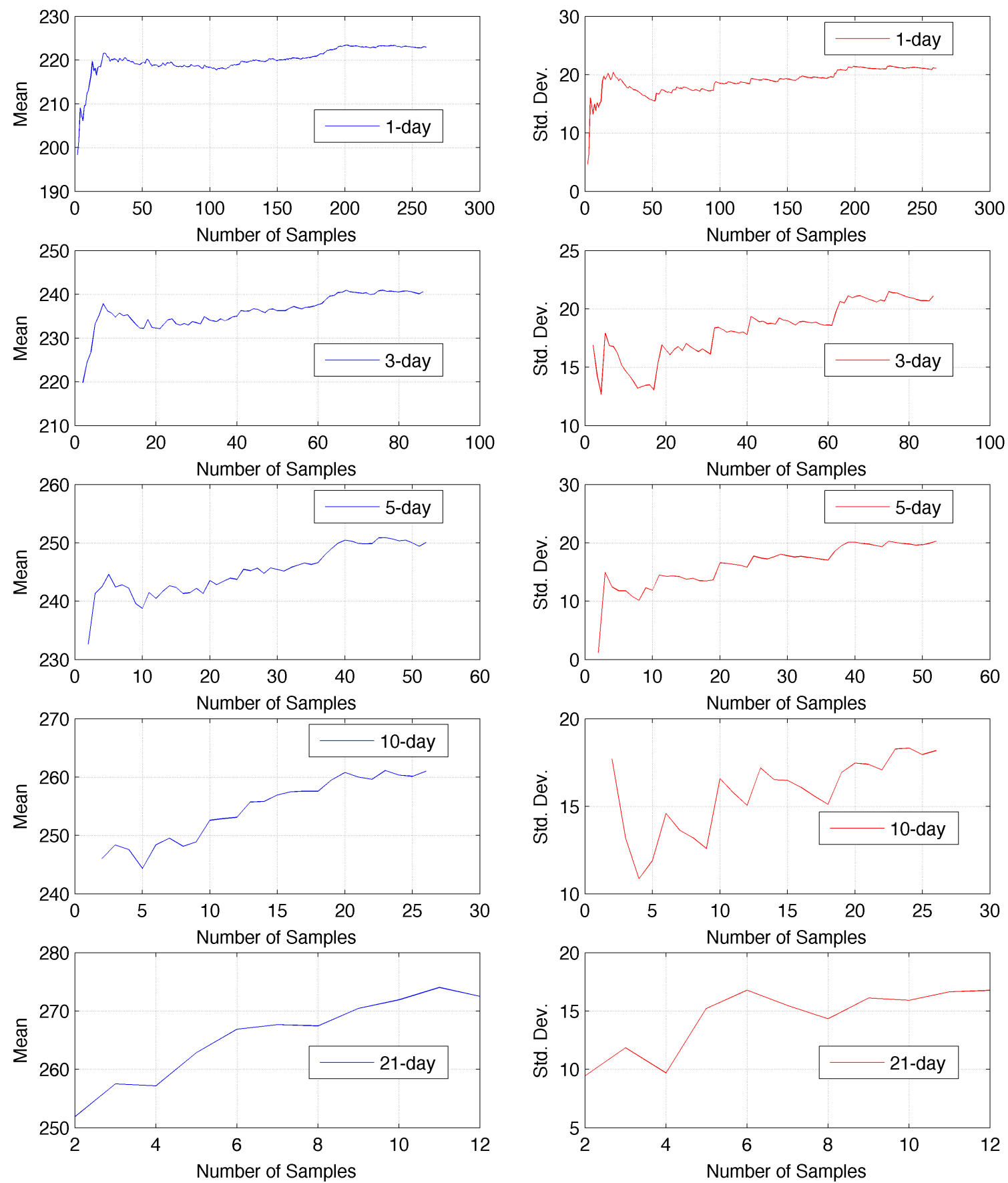

Figure 4.7.: Rolling mean and standard deviation for the censored data (weekends and holidays removed). 


\subsubsection{Transformation using Underlying Phenomenon Distribution}

For strategy shown in the last section, data from several days of monitoring is required however, in some cases, it may not be possible to obtain monitoring data for longer periods of time. The present section shows another strategy that can be applied to data obtained in shorter monitoring times. This methodology consists on approximating the distribution maximum live-load effect per vehicle (or loading event) and the total number of vehicles in a certain timeframe. By assuming that $X$ is the random variable that represents the maximum strain per vehicle passage and $n$ is the number of passages in a certain period, then the parameter $u_{n}$ of the EVD may be obtained through eq. 4.9. The shape parameter can then be obtained by a curve fitting method, such as least squares, by using 4.3 or 4.4 to evaluate sample points of the $Y_{n}$ distribution.

This methodology was applied to the data from the Jaguari River Bridge, due to the characteristics of the monitoring system, the data available was parsed in terms of loading events and not individual vehicle passages. The maximum strain due to each loading event was processed and organized, due to the mixed mode characteristic of this data, MATLAB's kernel density estimate was used to fit a distribution to the data. For illustration, Figure 4.8 shows the PDF and CDF which were fitted to the total data that was collected.
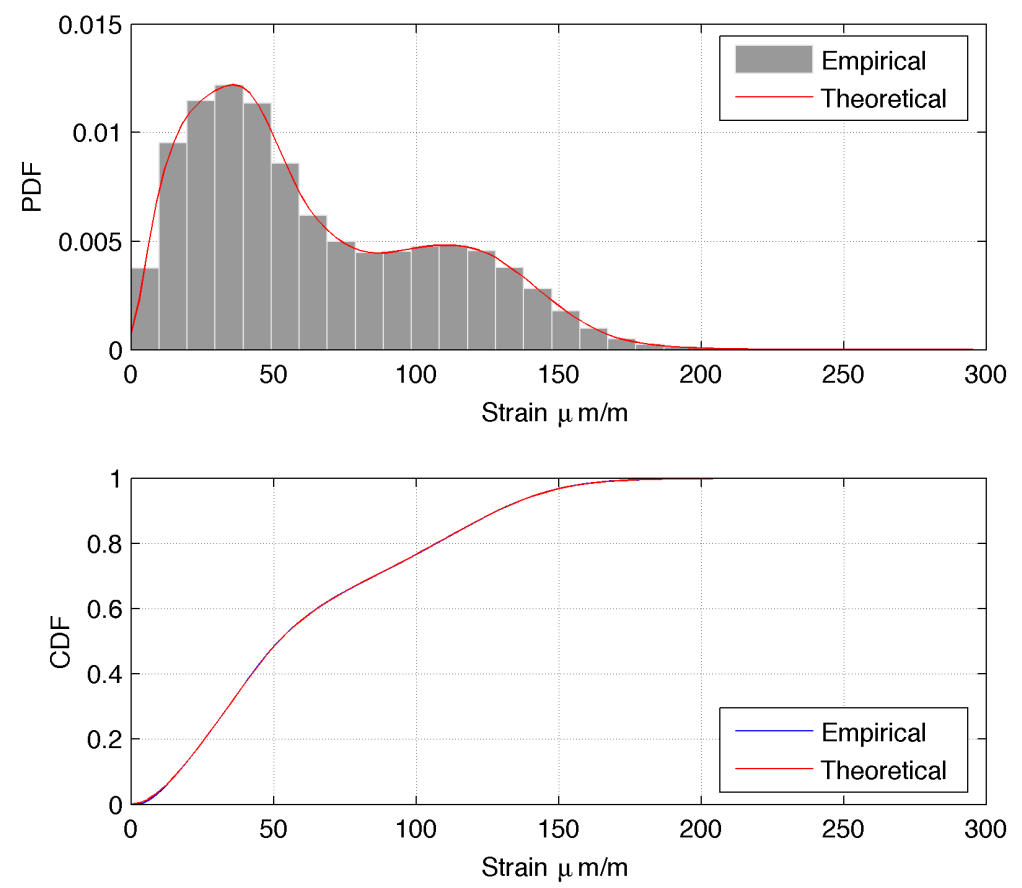

Figure 4.8.: Kernel density estimation of the maximum strain for the registered loading events for strain gauge E16.

The idea behind this approach is to estimate the value of $n$ in eqs. 4.9, 4.3 and 4.4 based 


\subsection{Live-Load Effect Characterization Using SHM Data}

on the number of observed loading events. Therefore, for a daily timeframe $n$ could be adopted as the average number of daily loading events and for a yearly timeframe $n$ would be 365 times that number. While using this value for the daily timeframe, as shown ahead, yields reasonable results, the extrapolation to the yearly timeframe can present issues. As mentioned in section 4.2.1, as $n$ grows eq. 4.3 tend to degenerate, therefore, care should be taken when using these equations to extrapolate with large values of $n$. To avoid this issue in this case study, the transformation is carried out to a daily timeframe and the yearly maximum distribution is then obtained by shifting the Gumbel distribution fitted to the extrapolated daily maximum data, as done in the previous section.

To better illustrate this procedure, the transformation will be carried out using the monitoring data collected in the Jaguari River Bridge. Again, based on the findings of the homogeneity tests, data collected during weekends and public holidays were removed from the dataset. The first step was to fit a kernel density estimate to the maximum strain per loading event data. By setting $n_{1 \text { day }}=2578.7$, which is the average number of daily loading events, and using eqs. 4.4 and 4.3 the PDF and CDF of the transformation from the kernel density estimate to the daily maximum strain EVD is obtained (shown as the red line with dots in Figure 4.9). The location parameter of the Gumbel EVD for the daily maximum strain, $u_{n_{1 d a y}}$, was then determined through eq. 4.9. The shape factor, $\alpha_{n_{1 d a y}}$, was determined by least squares using the transformed CDF data.

For comparison, Figure 4.9 shows the histograms of the recorded daily maximum data (based on the 260 days of observation) with a Gumbel fit to this data along with, the transformed EVD and the respective Gumbel fit based on this transformation. The same information is shown in terms of the CDF's, showing the empirical CDF (based on the daily maximum data) along with the Gumbel fit to the daily data and the transformed CDF with its respective Gumbel fit. These plots show that by using this transformation strategy it is possible to estimate with reasonable accuracy the daily maximum strain. In fact the mean and standard deviation of the obtained using this transformation are, respectively, 222.5 and 20.37 while, using MLE for the daily maximum data, respectively, 223.1 and 21.6 are obtained.

The next step is to use eq. 4.10 to transform the obtained Gumbel distribution to the yearly timeframe. In the same manner as done in section 4.2.2.2, for this transformation a total of 254 business days were considered in each year. The yearly maximum strain distributions that were obtained were very close to each other having mean values and standard deviations of $313.4 \mu \mathrm{m} / \mathrm{m}$ and $20.37 \mu \mathrm{m} / \mathrm{m}$, for the transformation based on the underlying and, $316.3 \mu \mathrm{m} / \mathrm{m}$ and $21.6 \mu \mathrm{m} / \mathrm{m}$ for the transformation based on the MLE for the daily maximum data. This result, which is illustrated in Figure 4.10, is expected since the Gumbel fits to the daily maximum data generated close distributions. 

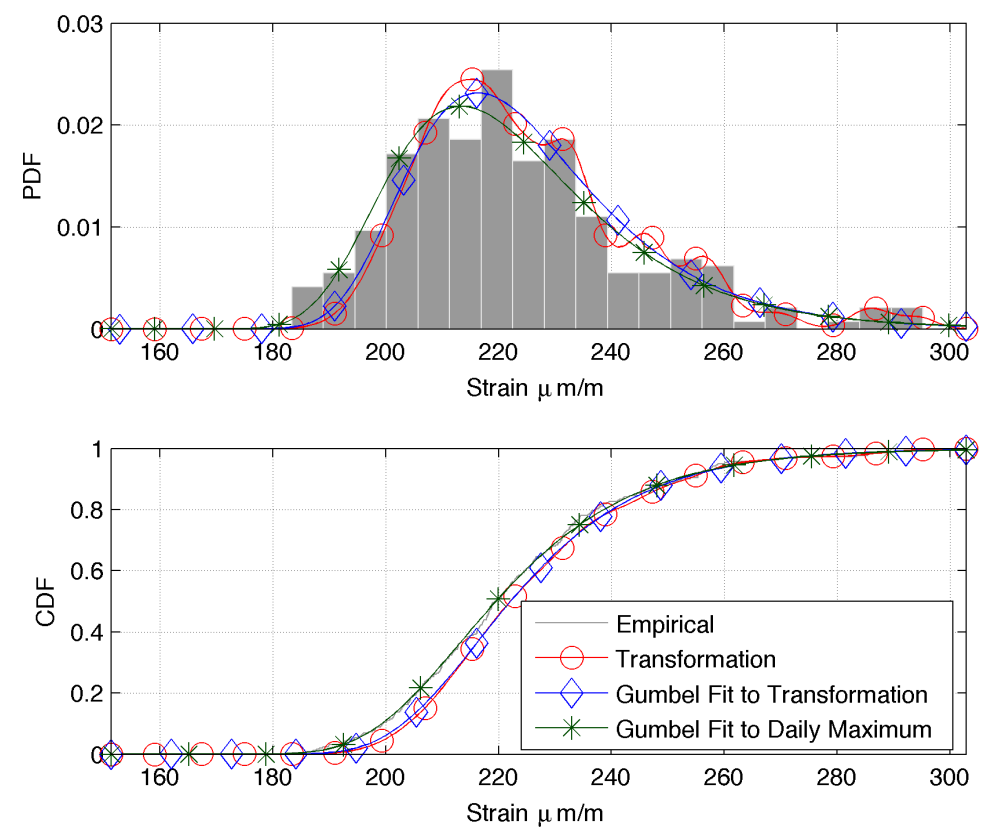

Figure 4.9.: Transformed 1-day EVD showing the empirical results, the exact transformation from the kernel density estimate, and the corresponding fitted Gumbel distribution for strain gauge E16.
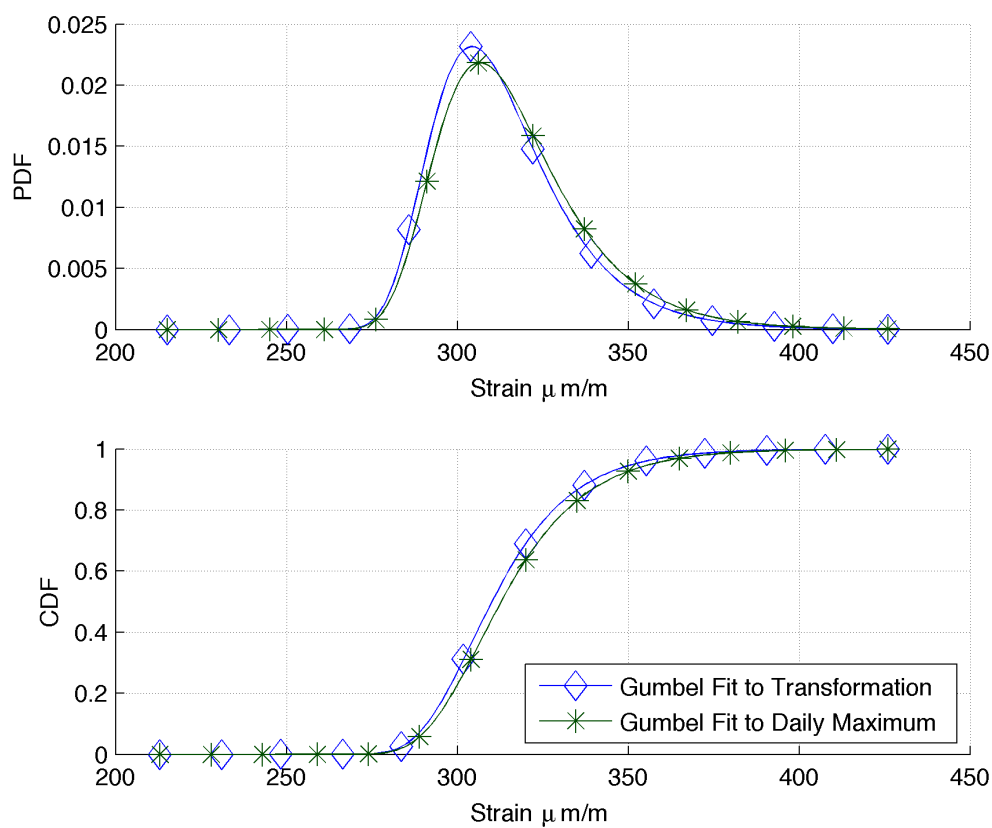

Figure 4.10.: Resulting PDF's and CDF's for yearly maximum strain distribution obtained by transforming from a Gumbel fit of the transformed underlying distribution and fit to the daily maximum data. 


\subsection{Life-Cycle Assessment of the Jaguari River Bridge Using SHM Data}

In the last sections, focus was given towards characterizing the live-load effects through SHM data. The focus of this final part of the chapter will be on the integration of this data to the probabilistic life-cycle analysis of the structure. For simplicity, only the flexural ultimate limit state of one of the cross-sections of the longitudinal girders was analyzed here. The corresponding limit state equation, given by eq. 4.11, is pretty straightforward, having the failure event, $g(X, t) \leq 0$, when the active bending moments, $M_{L I V E}(X)+M_{D E A D}(X)$, surpass the ultimate bending moment, $M_{U L T}(X, t)$.

$$
g(X, t)=M_{U L T}(X, t)-M_{L I V E}(X)-M_{D E A D}(X)
$$

The analyzed cross-section will be the outermost girder in Section S5 (see Figure 3.28). It was idealized as a $0.4 \mathrm{~m} \times 2.8 \mathrm{~m}$ rectangular cross-section with the reinforcement distribution as shown in Figure 4.11. The reinforcement was divided into groups according to the concrete cover values. In order to obtain the live load moment from the SHM data, a behavioral model based on Branson's formula[3] was used. Using the equivalent stiffness from Branson's formula the measured strains were then transformed into active bending moments. For this procedure, it was assumed that the structure did not present any damage at the time of monitoring.

\section{Section S5}

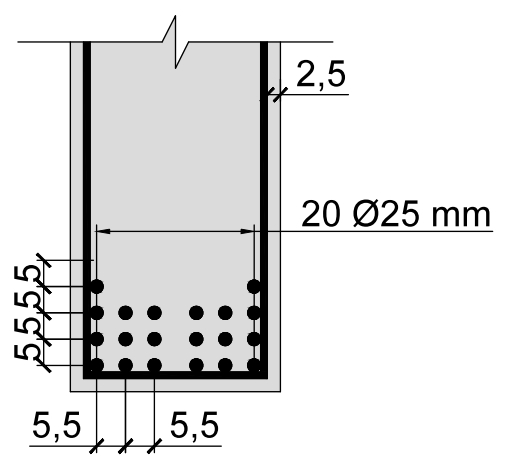

Figure 4.11.: Reinforcement distribution for the longitudinal girder at Section S5.

Using the measured live load response and the methodology from item 4.2.2.2 the yearly maximum live-load strain distribution was determined as a Gumbel distribution with mean and standard deviation of 309.6 and $18.8 \mu \mathrm{m} / \mathrm{m}$, respectively. A measurement uncertainty factor, $\gamma_{\varepsilon}$, was considered with a normal distribution mean one and c.o.v. of 
$5 \%$. This factor was multiplied to the live-load strain during the simulation of the active moment. Due to the nonlinear material behavior an estimate of the dead load moment acting on the section at the time of the monitoring was also considered. Table 4.3 presents the definition of all the random variables considered in this analysis. The parameters for the concrete strength were determined using the results from testing of concrete samples during the construction process which were available in the as-built documentation. For the carbonation constant distribution the data used for parameter estimation came from phenolphthalein testing.

\begin{tabular}{|c|c|c|c|c|c|}
\hline \multirow{2}{*}{$\begin{array}{c}\text { Model } \\
\text { Parameter }\end{array}$} & \multicolumn{3}{|c|}{ Distribution } & \multirow{2}{*}{ Unit } & \multirow{2}{*}{ Description } \\
\hline & Type & & arameters & & \\
\hline \multirow{2}{*}{$f_{c}$} & \multirow{2}{*}{ Lognormal } & 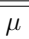 & 32.14 & \multirow{2}{*}{$\mathrm{MPa}$} & \multirow{2}{*}{ Concrete strength } \\
\hline & & $\sigma$ & 2.98 & & \\
\hline \multirow{2}{*}{$f_{\text {ctmo }}$} & \multirow{2}{*}{ Lognormal } & $\mu$ & 1.61 & \multirow{2}{*}{$\mathrm{MPa}$} & \multirow{2}{*}{ See eq. $(2.8)$} \\
\hline & & $\sigma$ & 0.322 & & \\
\hline \multirow{2}{*}{$E_{c 0}$} & \multirow{2}{*}{ Normal } & $\mu$ & $2.05 \times 10^{4}$ & \multirow{2}{*}{$\mathrm{MPa}$} & \multirow{2}{*}{ See eq. $(2.6)$} \\
\hline & & $\sigma$ & $1.64 \times 10^{3}$ & & \\
\hline \multirow{2}{*}{$f_{s y}$} & \multirow{2}{*}{ Lognormal } & $\mu$ & 560 & \multirow{2}{*}{$\mathrm{MPa}$} & \multirow{2}{*}{ Steel yield strength } \\
\hline & & $\sigma$ & 28.0 & & \\
\hline \multirow{2}{*}{$E_{s}$} & \multirow{2}{*}{ Normal } & $\mu$ & $2.1 \times 10^{5}$ & \multirow{2}{*}{$\mathrm{MPa}$} & \multirow{2}{*}{ Modulus of elasticity of steel } \\
\hline & & $\sigma$ & $6.93 \times 10^{3}$ & & \\
\hline \multirow{2}{*}{$c$} & \multirow{2}{*}{ Lognormal } & $\mu$ & 25 & \multirow{2}{*}{$\mathrm{mm}$} & \multirow{2}{*}{ Reinforcement cover } \\
\hline & & $\sigma$ & 8 & & \\
\hline \multirow{2}{*}{$K$} & \multirow{2}{*}{ Lognormal } & $\mu$ & 6.05 & \multirow{2}{*}{$\mathrm{mm} \cdot \mathrm{yr}^{-1 / 2}$} & \multirow{2}{*}{ Carbonation front constant } \\
\hline & & $\sigma$ & 2.19 & & \\
\hline \multirow{2}{*}{$\nu$} & \multirow{2}{*}{ Lognormal } & $\mu$ & $1.2 \times 10^{-3}$ & $\mathrm{~cm} \cdot \mathrm{vr}^{-1}$ & Corrosion rate \\
\hline & & $\sigma$ & $0.3 \times 10^{-3}$ & & Comtosion late \\
\hline$M_{D E A D}$ & Normal & $\mu$ & 1.05 & - & Dead load bending moment \\
\hline${ }^{M} D E A D$ & & $\sigma$ & 0.105 & & \\
\hline & Gumbel & $\mu$ & 309.6 & $\mu \mathrm{m} / \mathrm{m}$ & Yearly maximum live load strain \\
\hline Elive year & & $\sigma$ & 18.8 & $\mu \Pi 11111$ & 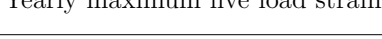 \\
\hline$\gamma_{\varepsilon}$ & Normal & $\mu$ & 1 & - & Measurement uncertainty \\
\hline IE & & $\sigma$ & 0.05 & & \\
\hline
\end{tabular}

Table 4.3.: Random variable definitions for the life-cycle analysis of cross-section S5.

Since the strength degradation due to reinforcement corrosion depends on the corrosion initiation time, it is convenient to calculate the yearly probability of failure according to eq. 4.12. The integral in this equation was solved by applying the Monte Carlo method with 1,000 samples, Figure 4.12 shows the sampled output along with the fitted lognormal distribution for $T_{i}$.

$$
P_{f_{\text {yearly }} i}=\int_{z} P_{f_{y} i}(X \mid z) \cdot f_{T_{i}}(z) d z
$$

In this case, the First Order Reliability Method (FORM) [56] was used to determine $P_{f_{y} i}(X \mid z)$, which is the yearly probability of failure conditional to a given initiation time $z$. The time-dependent reliability of the structure may be approximated using the yearly probabilities of failure using eq. 4.13 as suggested in [94, 56, 95]. Figure 4.13(a) shows the annual reliability indices (associated with the yearly probability of failure) throughout the 


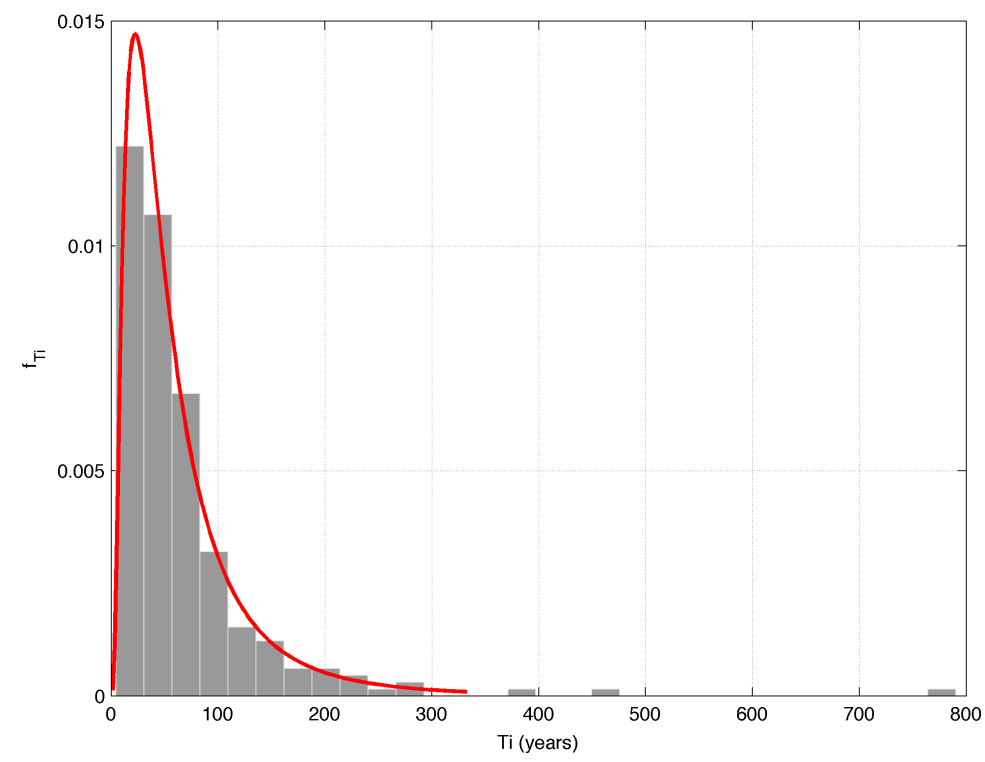

Figure 4.12.: Simulated corrosion initiation time along with the fitted PDF.

cross-section's lifetime and Figure 4.13(b) shows the estimated time dependent reliability for the analyzed cross-section.

$$
P_{f_{t}}=1-\prod_{i=1}^{t}\left[1-P_{f_{\text {yearly }} i}\right]
$$

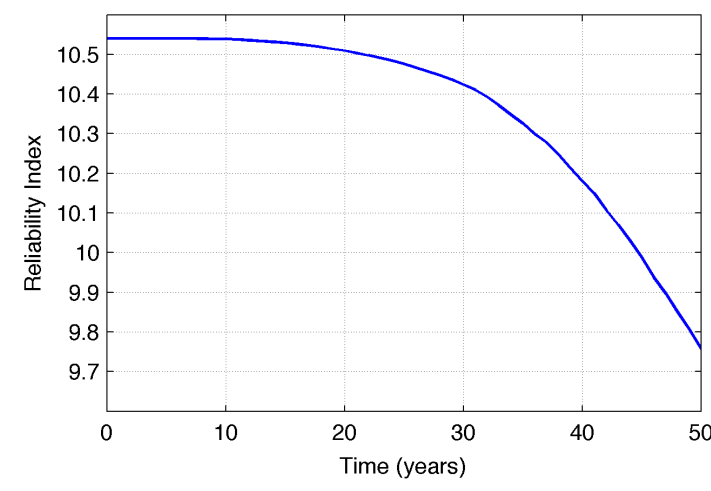

(a)

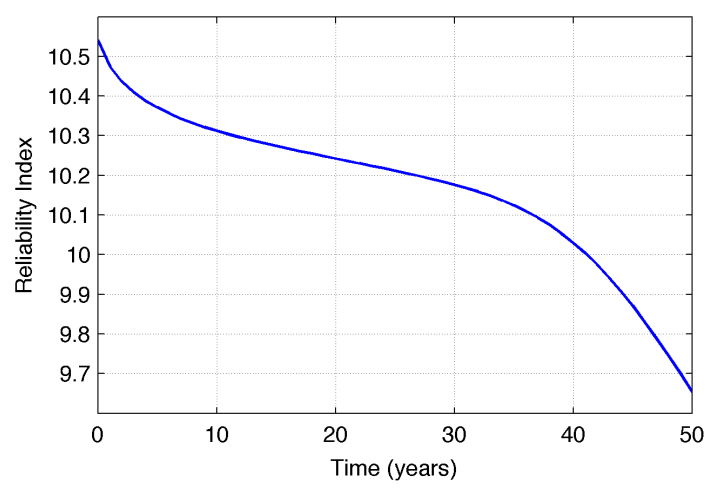

(b)

Figure 4.13.: Plots showing: (a) annual reliability indices; (b) time-dependent reliability indices.

From these results it can be seen that a very high level of reliability is associated with the flexural mode of failure when the live-loading is characterized through monitoring. These reliability values most likely indicate that the live-load model used in the design is very conservative for this structural type. In continuos bridges such as this one, the 


\subsection{Final Considerations}

design truck and the crowd loading are positioned in such a pattern as to maximize the loading at the analyzed section. Which is a condition that was not detected during the monitoring period. While this is certainly one of the reasons behind these results, it may not be the only one. However, during the load testing shown in Chapter 3 relatively low strains were recorded, this indicates that the safety level of the structure is relatively high.

Regarding the lifetime reliability profiles, they indicate that through the analyzed years an adequate safety level is maintained. As mentioned, the field data from the carbonation depth measurements were used to update the parameters of the carbonation constant $(K)$ however, no information regarding the corrosion rate was taken into consideration. Therefore, in these results, the rate at which the reliability decreases is based solely on the corrosion rate established based on the available literature which, in this case, was based on a corrosion current density $i_{\text {corr }}=1 \mu \mathrm{A} / \mathrm{cm}^{2}[95,88,87]$. Having said this, it is recommended that, at the point in the near future where this model indicates that corrosion is probable to initiate, additional inspections should be carried out in order to evaluate the current state. Monitoring during future stages of this degradation process could give a better idea regarding the rate at which this degradation occurs.

\subsection{Final Considerations}

This chapter takes into consideration some aspects of integrating SHM data into life-cycle assessment of structures. The use of the measured structural response to determine the live-load model for structural assessment is an interesting use of SHM data. The load models for structural design must take into consideration data from weight stations as well as traffic flow behavior over the bridge. This demands simulation of with different axle loads and combinations. Therefore, the use of the site specific monitored live-load response can lead to significant improvement in the assessment process.

Concepts of extreme value theory were applied to determine the probabilistic models for the live-loads. In particular the interest was towards determining the yearly maximum live-load effect distribution. Two methods were applied, the first which used data processed for maximum values during different observation timeframes and another which used data processed for maximum strain due to individual loading events. It was demonstrated that in order to obtain consistency in the resulting transformation to the yearly timeframe, homogeneity of the collected data is important. Both methodologies gave compatible results indicating that they can be used for the purpose of determining live-load effect distribution.

In this chapter the life-cycle assessment of RC structures was also taken into consideration. In most of the consulted literature, mentioned in section 4.1.1, the applications have been 
limited to steel structures. Generally the measured strains from SHM are transformed into stresses and a limit state equation is formulated in terms of the acting stresses and a stress limit. Due to the nonlinear behavior of reinforced concrete this straightforward approach is not feasible. Requiring the use of adequate behavioral models in order to incorporate the measurements into a safety assessment. Branson's formula was used in this case to transform the measured strain information into active forces (bending moments). A timedependent reliability analysis was carried out with this information which indicated that, based on the monitoring results, the structure is in excellent state. 


\section{Conclusion and Future Works}

\subsection{Field Testing and Life-Cycle Assessment}

Structural field testing has proven to be a powerful tool for condition assessment. The dataset composed of structural response and loading is very useful for model updating. A lot of work in this direction has been done with steel structures, where the linear behavior allows for extrapolations regarding the ultimate state behavior of the structure. For RC structures this approach is not as straightforward, as the stiffness may change during loading, due to cracking, changing the load distribution characteristic. Therefore, care must be taken when using testing data in these structures.

In chapter 2 an approach that uses the correlation between the concrete strength and stiffness was tested. The correlation between these parameters was established through a series of constitutive models in a sectional analysis. Significant changes in the predicted strength were obtained with this methodology, this was mainly due to a much stiffer response than predicted. A bias factor was used in the likelihood function formulation in order to account for increases in stiffness that were not considered by the model, such as, support stiffness and railway track interaction. It should be noted that this behavior was found on other RC railway bridges tests which the author worked on.

Also, a fairly large measurement error was considered during these tests. This was due to uncertainties in the weigh-in-motion system installed in the rails. Testing of the algorithm with smaller measurement errors in the likelihood function resulted in better fit between the predicted and measured response and greater reduction in parameter uncertainty. These results were consistent with the review literature. However, the idea behind the example shown in the thesis was to show the potential issues that are present in practical applications.

The updating considering test done at different ages of the structure demonstrated that the methodology can be used to obtain better estimates of the degradation mechanisms. However, for the considered case study, the testing data improved the predicted response before and at the time of testing. The method in this case was not very effective in improving the prognosis of the behavior. This is due to the larger measurement errors which allows for more flexibility in the adjusted parameters. Resulting in a poor fit of 
the modeled lifetime behavior. Bayesian updating has been successfully applied to time dependent problems, however not considering large multiplicative errors as was the case.

Finally some suggestions for future works in this area include:

- Use of more advanced modeling techniques including better material models and inclusion of stiffness contributions from track and bridge bearings;

- Development of optimal testing intervals considering minimizing costs and maximizing damage detection capability;

- Application of weight functions to increase the influence of the data sets.

\subsection{Structural Health Monitoring of the Jaguari River Bridge}

One of the most important contributions of the work done in this thesis is the structural monitoring system installed in the Jaguari River Bridge. The experience and data collected by this system will benefit the research of several current and future members of the research group. Characteristics of this system, such as the use of two different monitoring systems, with different sensor technologies, allows for the evaluation of the long term behavior of these systems.

As more data becomes available, some of the evaluations shown here, such as the EVD characterization, can be reevaluated. With a bigger dataset more research towards determining sufficient monitoring times can be made. Also, with the installation of a Weighin-Motion station located a few kilometers from this bridge, correlation studies between these datasets can be made. At last, the processing of the captured images from the traffic camera is also a possibility.

\subsection{Use of SHM Data in the Life-Cycle Assessment}

An approach for using SHM data in the life-cycle assessment with RC was demonstrated. Measured strains were inputed into a sectional analysis model to determine the distribution of the live-load moment. Degradation due to corrosion of the reinforcing steel was also considered in the sectional analysis model. The results obtained in the time dependent analysis were satisfactory and showed the bridge to be in good state of conservation. These results are consistent with the findings of the bridge's inspection and testing.

Promising research topics in this area include the analysis of the effect of uniform and non-uniform damage in the load distribution characteristics of RC continuous beams. 
Research on damage detection using SHM has focused mainly on frequency data from accelerometers. Using the fact that changes in stiffness result in changes in natural frequency. When monitoring strains detecting changes in stiffness can be difficult since it is difficult to relate change in output to damage or load increase. In continuous beams SHM may be able to capture changes in stiffness due to the change in the load distribution characteristics. This is a promising approach to be pursued. 


\section{Bibliography}

[1] AASHTO. Standard specification for highway bridges. 2002.

[2] ABNT. NBR 12655: Portland cement concrete - Preparation, control, receipt and acceptance - Procedure, 2015.

[3] ACI Committee 318. Building Code Requirements for Structural Concrete ( ACI 318-08 ), volume 2007. 2008.

[4] Alfredo H-S. Ang and Wilson H. Tang. Probability concepts in engineering planning and design, Vol. II. Wiley, New York, 1984.

[5] Alfredo H-S. Ang and Wilson H. Tang. Probability Concepts in Engineering. John Wiley \& Sons, Inc., 2nd editio edition, 2007.

[6] Siu-Kui Au and James L. Beck. Estimation of small failure probabilites in high dimensions by subset simulation. Probabilistic Engineering Mechanics, 16:263-277, 2001.

[7] Baidar Bakht and Leslie G. Jaeger. Bridge Testing-A Surprise Every Time. Journal of Structural Engineering, 116(5):1370-1383, may 1990.

[8] Giorgio Barone, Dan M. Frangopol, and Mohamed Soliman. Optimization of LifeCycle Maintenance of Deteriorating Bridges with Respect to Expected Annual System Failure Rate and Expected Cumulative Cost. Journal of Structural Engineering, 140(2):04013043-1-13, feb 2014.

[9] James L. Beck and Siu-Kui Au. Bayesian Updating of Structural Models and Reliability using Markov Chain Monte Carlo Simulation. Journal of Engineering Mechanics, 128(No. 4):380-391, 2002.

[10] James L. Beck and L. S. Katafygiotis. Updating Models and Their Uncertainties. I: Bayesian Statistical Framework. Journal of Engineering Mechanics, 124(No. 4):455461, 1998.

[11] James L. Beck and Ka-Veng Yuen. Model selection using response measurements: Bayesian probabilistic approach. Journal of Engineering Mechanics, 130(2):192203, 2004 . 
Bibliography

[12] Wolfgang Betz, Iason Papaioannou, and Daniel Straub. Adaptive variant of the BUS approach to Bayesian updating. In 9th International Conference on Structural Dynamics (EURODYN), 2014.

[13] Enrique Castillo. Extreme value theory in engineering. Academic Press, 1988.

[14] F. Necati Catbas, Melih Susoy, and Dan M. Frangopol. Structural health monitoring and reliability estimation: Long span truss bridge application with environmental monitoring data. Engineering Structures, 30(9):2347-2359, sep 2008.

[15] CEB-FIP. CEB-FIP Model Code 2010. Technical report, Comite euro-international du beton, London, England, 2011.

[16] Michael J. Chajes, Dennis R. Mertz, and Brett Commander. Experimental Load Rating of a Posted Bridge. Journal of Bridge Engineering, 2(1):1-10, 1997.

[17] Sai Hung Cheung and James L. Beck. Bayesian Model Updating Using Hybrid Monte Carlo Simulation with Application to Structural Dynamic Models with Many Uncertain Parameters. Journal of Engineering Mechanics, 135(4):243-255, 2009.

[18] Michael P. Collins and Denis Mitchell. Prestressed concrete structures. Response Publications, 1997.

[19] Robert J. Connor and Justin R. Mccarthy. Report on Field Measurements and Uncontrolled Load Testing of the Lehigh River Bridge ( SR-33 ). Technical report, ATLSS Reports, ATLSS Reports, 2006.

[20] Robert J. Connor and Brian J. Santosuosso. Field Measurements and Controlled Load Testing on the Lehigh River Bridge (SR-33). Technical report, ATLSS Reports, ATLSS Reprts, 2002.

[21] Ove Ditlevsen and Henrik O. Madsen. Structural Reliability Methods. Wiley, New York, 1996.

[22] Tamer El Maaddawy, Khaled Soudki, and Timothy Topper. Analytical Model to Predict Nonlinear Flexural Behavior of Corroded Reinforced Concrete Beams. ACI Structural Journal, 102(No. 4):550-559, 2005.

[23] Bruce R. Ellingwood. Reliability-based condition assessment and LRFD for existing structures. Structural Safety, 18(2-3):67-80, jan 1996.

[24] Michael P. Enright and Dan M. Frangopol. Condition Prediction of Deteriorating Concrete Bridges Using Bayesian Updating. Journal of Structural Engineering, 125(No. 10):1118-1125, 1999.

[25] Allen C. Estes and Dan M. Frangopol. Updating Bridge Reliability Based on Bridge Management Systems Visual Inspection Results. Journal of Bridge Engineering, 8(December):374-382, 2003. 
Bibliography

[26] Michael Havbro Faber, Dimitri V. Val, and Mark G. Stewart. Proof load testing for bridge assessment and upgrading. Engineering Structures, 22(12):1677-1689, dec 2000.

[27] Daniel Fink. A Compendium of Conjugate Priors. Montana The Magazine Of Western History, (1994):1-47, 1997.

[28] Dan M. Frangopol. Life-cycle performance, management, and optimisation of structural systems under uncertainty: accomplishments and challenges. Structure and Infrastructure Engineering, 7(6):389-413, jun 2011.

[29] Dan M. Frangopol, Jung S. Kong, and Emhaidy S. Gharaibeh. Reliability-Based Life-Cycle Management of Highway Bridges. Journal of Computing in Civil Engineering, 15(January):27-34, 2001.

[30] Dan M. Frangopol, Kai-Yung Lin, and Allen C. Estes. Reliability of Reinforced Concrete Girders under Corrosion Attack. Journal of Structural Engineering, 123(March):286-297, 1997.

[31] Dan M. Frangopol and Min Liu. Maintenance and management of civil infrastructure based on condition, safety, optimization, and life-cycle cost. Structure and Infrastructure Engineering, 3(1):29-41, mar 2007.

[32] Dan M. Frangopol, Alfred Strauss, and Sunyong Kim. Bridge Reliability Assessment Based on Monitoring. Journal of Bridge Engineering, 13(3):258-270, may 2008.

[33] Dan M. Frangopol, Alfred Strauss, Sunyong Kim, Dan M. Frangopol, and Sunyong Kim. Use of monitoring extreme data for the performance prediction of structures: Bayesian updating. Engineering Structures, 30(12):3654-3666, dec 2008.

[34] W. Brent Hall and Maolin Tsai. Load testing, structural reliability and test evaluation. Structural Safety, 6:285-302, 1989.

[35] A. Herwig. Reinforced concrete bridges under increased railway traffic loads -fatigue behaviour and safety measures. PhD thesis, École Polytechnique Fédérale de Lausanne, 2008.

[36] D. Hosser, C. Klinzmann, and R. Schnetgöke. A framework for reliability-based system assessment based on structural health monitoring. Structure and Infrastructure Engineering, 4(4):271-285, aug 2008.

[37] International Federation for Structural Concrete Task Group 5.6 Model Code for Service Life Design of Concrete Structures. Model code for service life design. Number 34. International Federation for Structural Concrete (fib), 2006.

[38] E T Jaynes. Where Do We Stand on Maximum Entropy? In R. D. Levine and M. Tribus, editors, The Maximum Entropy Formalism, pages 15-118, Cambridge, Mass - USA, 1978. MIT Press. 
Bibliography

[39] Sylvia Keßler, Johannes Fischer, Daniel Straub, and Christoph Gehlen. Updating of service-life prediction of reinforced concrete structures with potential mapping. Cement and Concrete Composites, 47:47-52, mar 2014.

[40] Sunyong Kim. Integrated Life-Cycle Framework for Optimal Inspection, Monitoring and Maintenance under Uncertainty: Applications to Highway Bridges and Naval Ship Structures. Phd thesis, Lehigh University, 2011.

[41] Sunyong Kim and Dan M. Frangopol. Optimal planning of structural performance monitoring based on reliability importance assessment. Probabilistic Engineering Mechanics, 25(1):86-98, jan 2010.

[42] Sunyong Kim and Dan M. Frangopol. Cost-Effective Lifetime Structural Health Monitoring Based on Availability. Journal of Structural Engineering, 137(1):22-33, 2011.

[43] Sunyong Kim, Dan M. Frangopol, and Mohamed Soliman. Generalized Probabilistic Framework for Optimum Inspection and Maintenance Planning. Journal of Structural Engineering, 139(March):435-447, 2013.

[44] Jung S. Kong and Dan M. Frangopol. Life-Cycle Reliability-Based Maintenance Cost Optimization of Deteriorating Structures with Emphasis on Bridges. Journal of Structural Engineering, 129(6):818-828, jun 2003.

[45] S Kotz and S Nadarajah. Extreme Value Distributions: Theory and Applications. Imperial College Press, 2000.

[46] S. S. Law and J. Li. Updating the reliability of a concrete bridge structure based on condition assessment with uncertainties. Engineering Structures, 32(1):286-296, jan 2010.

[47] Min Liu and Dan M. Frangopol. Optimal bridge maintenance planning based on probabilistic performance prediction. Engineering Structures, 26(7):991-1002, jun 2004.

[48] Ming Liu, Dan M. Frangopol, and Sunyong Kim. Bridge Safety Evaluation Based on Monitored Live Load Effects. Journal of Bridge Engineering, 14(4):257-269, 2009.

[49] Ming Liu, Dan M. Frangopol, and Sunyong Kim. Bridge System Performance Assessment from Structural Health Monitoring : A Case Study. Journal of Structural Engineering, 135(6):733-742, 2009.

[50] Yafei Ma, Lei Wang, Jianren Zhang, Yibing Xiang, and Yongming Liu. Bridge Remaining Strength Prediction Integrated with Bayesian Network and In Situ Load Testing. Journal of Bridge Engineering, 19(No. 10):1-11, 2014.

[51] Yafei Ma, Jianren Zhang, Lei Wang, and Yongming Liu. Probabilistic prediction 
Bibliography

with bayesian updating for strength degradation of RC bridge beams. Structural Safety, 44:102-109, 2013.

[52] James G. Macgregor, Sher Ali Mirza, and Bruce R. Ellingwood. Statistical Analysis of Resistance of Reinforced and Prestressed Concrete Members. ACI Journal, 80(3):167-176, 1983.

[53] Marc A. Maes. Updating Performance and Reliability of Concrete Structures Using Discrete Empirical Bayes Methods. Journal of Offshore Mechanics and Arctic Engineering, 124(4):239, 2002.

[54] Philip S. Marsh and Dan M. Frangopol. Lifetime Multiobjective Optimization of Cost and Spacing Reinforced Concrete Bridge Deck. Journal of Structural Engineering, 133(6):777-787, 2007.

[55] Philip S. Marsh and Dan M. Frangopol. Reinforced concrete bridge deck reliability model incorporating temporal and spatial variations of probabilistic corrosion rate sensor data. Reliability Engineering 63 System Safety, 93(3):394-409, mar 2008.

[56] Robert E. Melchers. Structural Reliability Analysis and Prediction. Wiley, 2nd edition, 1999.

[57] Thomas B. Messervey. Integration of Structural Health Monitoring into the Design , Assessment, and Management of Civil Infrastructure. Phd thesis, University of Pavia, 2009.

[58] Thomas B. Messervey, Dan M. Frangopol, and Sara Casciati. Application of the statistics of extremes to the reliability assessment and performance prediction of monitored highway bridges. Structure and Infrastructure Engineering, 7(1-2):8799, jan 2011.

[59] Nicholas Metropolis, Arianna W. Rosenbluth, Marshall N. Rosenbluth, Augusta H. Teller, and Edward Teller. Equation of State Calculations by Fast Computing Machines. The Journal of Chemical Physics, 21(6):1087, 1953.

[60] Sher Ali Mirza, Michael Hatzinikolas, and James G. MacGregor. Statistical Descriptions of Strength of Concrete. Journal of the Structural Division, 105(ST6):10211032, 1979.

[61] Sher Ali Mirza and James G. MacGregor. Variability of Mechanical Properties of Reinforcing Bars. Journal of the Structural Division, 105(ST5):921-937, 1979.

[62] Sher Ali Mirza and James G. MacGregor. Variations in Dimensions of Reinforced Concrete Members. Journal of the Structural Division, 105(ST4):751-766, 1979.

[63] Yasuhiro Mori and Bruce R. Ellingwood. Methodology for reliability based condition assessment. Application to concrete structures in nuclear plants. Technical 
Bibliography

report, Nuclear Regulatory Commission, Washington, DC (United States). Div. of Engineering; Oak Ridge National Lab., TN (United States); Johns Hopkins Univ., Baltimore, MD (United States). Dept. of Civil Engineering, 1993.

[64] Fred Moses, Jean Paul Lebet, and Rolf Bez. Applications of Field Testing to Bridge Evalution. Journal of Structural Engineering, 120(6):1745-1762, 1994.

[65] Hani Nassif and Mayrai Gindy. Effect of bridge live load based on 10 years of WIM data. In 3rd International conference on bridge maintenance and safety, 2008.

[66] Luís C. Neves, Dan M. Frangopol, and Paulo J. S. Cruz. Probabilistic LifetimeOriented Multiobjective Optimization of Bridge Maintenance: Single Maintenance Type. Journal of Structural Engineering, 132(No. 6):991-1005, 2006.

[67] Y Q Ni, X W Ye, and J M Ko. Modeling of Stress Spectrum Using Long-Term Monitoring Data and Finite Mixture Distributions. Journal of Engineering Mechanics, 138(February):175-183, 2012.

[68] Andrzej S. Nowak and T. Tharmabala. Bridge Reliability Evaluation Using Load Tests. Journal of Structural Engineering, 114(10):2268-2279, 1988.

[69] Andrzej S. Nowak, Ahmed S. Yamani, and Sami W. Tabsh. Probabilistic Models for Resistance of Concrete Bridge Girders. ACI Structural Journal, 91(No. 3):269-276, 1994.

[70] Eugene J. O’Brien, F. Schmidt, D. Hajializadeh, X. Y. Zhou, Bernard Enright, Colin C. Caprani, S. Wilson, and E. Sheils. A review of probabilistic methods of assessment of load effects in bridges. Structural Safety, 53:44-56, 2015.

[71] Nader M. Okasha and Dan M. Frangopol. Lifetime-oriented multi-objective optimization of structural maintenance considering system reliability, redundancy and life-cycle cost using GA. Structural Safety, 31(6):460-474, nov 2009.

[72] Nader M. Okasha and Dan M. Frangopol. Novel Approach for Multicriteria Optimization of Life-Cycle Preventive and Essential Maintenance of Deteriorating Structures. Journal of Structural Engineering, 136(No. 8):1009-1022, 2010.

[73] Nader M. Okasha and Dan M. Frangopol. Integration of structural health monitoring in a system performance based life-cycle bridge management framework. Structure and Infrastructure Engineering, 8(No. 11):999-1016, jun 2012.

[74] Nader M. Okasha, Dan M. Frangopol, and André D. Orcesi. Automated finite element updating using strain data for the lifetime reliability assessment of bridges. Reliability Engineering \& System Safety, 99:139-150, mar 2012.

[75] Piotr Olaszek, Marek Lagoda, and Joan Ramon Casas. Diagnostic load testing and assessment of existing bridges: examples of application. Structure and Infrastructure Engineering, (July 2013):1-9, mar 2013. 
Bibliography

[76] André D. Orcesi and Dan M. Frangopol. Inclusion of Crawl Tests and Long-Term Health Monitoring in Bridge Serviceability Analysis. Journal of Bridge Engineering, 15(3):312-326, may 2010.

[77] André D. Orcesi and Dan M. Frangopol. Optimization of bridge maintenance strategies based on structural health monitoring information. Structural Safety, 33(1):2641, jan 2011

[78] André D. Orcesi and Dan M. Frangopol. Bridge Performance Monitoring Based on Traffic Data. Journal of Engineering Mechanics, 139(November):1508-1520, 2013.

[79] Iason Papaioannou, Kilian Zwirglmaier, and Daniel Straub. Assessment of MCMC Algorithms for Subset Simulation. (2):1-34, 2013.

[80] Frédéric Perrin and Bruno Sudret. Comparison of Markov chain Monte-Carlo simulation and a FORM-based approach for Bayesian updating of mechanical models. In 10th Int. Conf on Applications of Stat. and Prob. in Civil Engineering, pages 1-8, Tokyo, Japan, 2007.

[81] Frédéric Perrin, Bruno Sudret, and Maurice Pendola. Bayesian updating of mechanical models - Application in fracture mechanics. In 18ème Congrès Français de Mécanique, pages 27-31, Grenoble, France, 2007.

[82] M.Imran Rafiq. Health Monitoring in Proactive Reliability Management of Deteriorating Concrete Bridges. Phd, University of Surrey, 2005.

[83] M.Imran Rafiq, Marios K. Chryssanthopoulos, and Toula Onoufriou. Performance updating of concrete bridges using proactive health monitoring methods. Reliability Engineering 83 System Safety, 86(3):247-256, dec 2004.

[84] A. J. M. Siemes, A. C. W. M. Vrouwenvelder, and A. van den Beukel. Durability of buildings: A reliability analysis. Heron, 30(3):1-48, 1985.

[85] Mohamed Soliman, Dan M. Frangopol, and Sunyong Kim. Probabilistic optimum inspection planning of steel bridges with multiple fatigue sensitive details. Engineering Structures, 49:996-1006, apr 2013.

[86] Mohamed Soliman, Dan M. Frangopol, and Kihyon Kown. Fatigue Assessment and Service Life Prediction of Existing Steel Bridges by Integrating SHM into a Probabilistic Bilinear S - N Approach. Journal of Structural Engineering, 139(No. 10):1728-1740, 2013.

[87] Mark G. Stewart and David V. Rosowsky. Structural Safety and Serviceability of Concrete Bridges Subject to Corrosion. Journal of Infrastructure Systems, 4(4):146155, 1998.

[88] Mark G. Stewart and David V. Rosowsky. Time-dependent reliability of deteriorating reinforced concrete bridge decks. Structural Safety, 20:91-109, 1998. 
[89] Daniel Straub. Reliability updating with equality information. Probabilistic Engineering Mechanics, 26(2):254-258, 2011.

[90] Daniel Straub and Iason Papaioannou. Bayesian Updating with Structural Reliability Methods. Journal of Engineering Mechanics, 141(3):04014134, 2014.

[91] V. S. Sundar and C. S. Manohar. Updating reliability models of statically loaded instrumented structures. Structural Safety, 40:21-30, 2013.

[92] Qinghui Suo and Mark G. Stewart. Corrosion cracking prediction updating of deteriorating RC structures using inspection information. Reliability Engineering 8 System Safety, 94(8):1340-1348, aug 2009.

[93] P. Thoft-Christensen, F. M. Jensen, C. R. Middleton, and A. Blackmore. Assessment of the Reliability of Concrete Slab Bridges. In 7th IFIP WG 7.5 Working Conference, Boulder, Colorado, USA, 1996.

[94] Dimitri V. Val and Robert E. Melchers. Reliability of Deteriorating RC Slab Bridges. Journal of Structural Engineering, 123(No. 12):1638-1644, 1997.

[95] Dimitri V. Val, Mark G. Stewart, and Robert E. Melchers. Life-cycle performance of RC bridges: probabilistic approach. Computer-Aided Civil and Infrastructure Engineering, 15(1):14-25, 2000.

[96] M. W. Vanik, James L. Beck, and Siu-Kui Au. Bayesian Probabilistic Approach to Structural Health Monitoring. Journal of Engineering Mechanics, 126(No. 7):738745,2000 .

[97] Frank J. Vecchio and Michael P. Collins. The modified compression-field theory for reinforced concrete elements subjected to shear, 1986.

[98] Naiyu Wang, Curtis O'Malley, Bruce R. Ellingwood, and Abdul-Hamid Zureick. Bridge Rating Using System Reliability Assessment . I : Assessment and Verification by Load Testing. Journal of Bridge Engineering, 16(No. 6):854-862, 2011.

[99] Ka-Veng Yuen, James L. Beck, and Lambros S. Katafygiotis. Unified Probabilistic Approach for Model Updating and Damage Detection. Journal of Applied Mechanics, 73(4):555, 2006.

[100] Y. Zhou, B. Gencturk, K. Willam, and A. Attar. Carbonation-Induced and Chloride-Induced Corrosion in Reinforced Concrete Structures. Journal of Materials in Civil Engineering, pages 04014245-1 - 04014245-17, 2014.

[101] Benjin Zhu and Dan M. Frangopol. Reliability assessment of ship structures using Bayesian updating. Engineering Structures, 56:1836-1847, nov 2013.

[102] Konstantin M. Zuev, James L. Beck, Siu-Kui Au, and Lambros S. Katafygiotis. Bayesian post-processor and other enhancements of Subset Simulation for estimat- 
Bibliography

ing failure probabilities in high dimensions. Computers and Structures, 92-93:283$296,2012$. 
Appendices 


\section{A. Collected Data from the Monitoring of the Jaguari River Bridge}

\section{A.1. Introduction}

In this appendix a collection of the data obtained during the testing, short-term monitoring and long-term monitoring of the Jaguari River is shown.

\section{A.2. Load-Test Results}

The strain measurements from the load-tests are shown in this section. Due to slippage of some of the displacement sensors moving end from the reference plate, the displacement were not recorded correctly in all the spans e will not be shown here. 

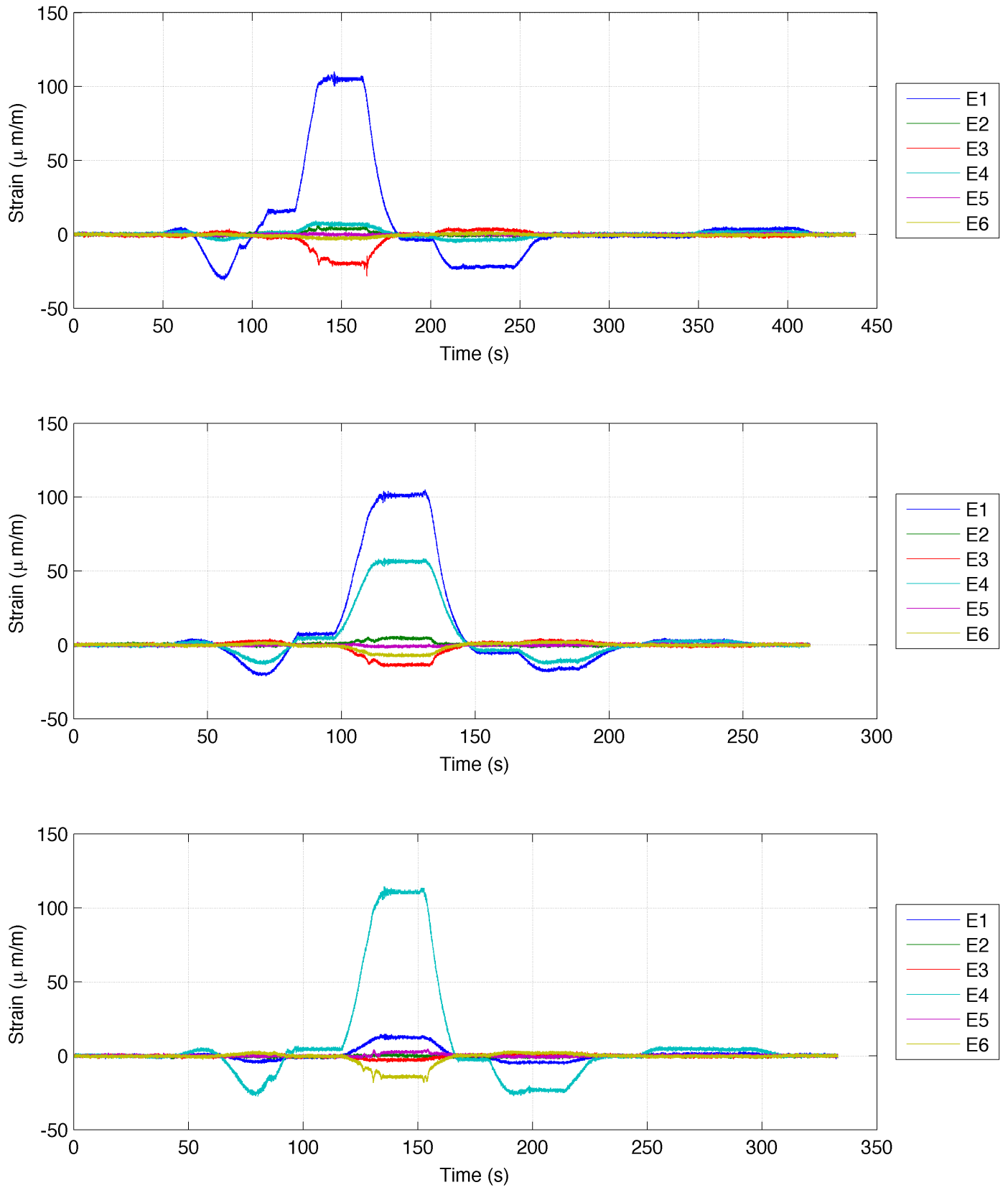

Figure A.1.: Recorded strains in the static load test in section S1. 

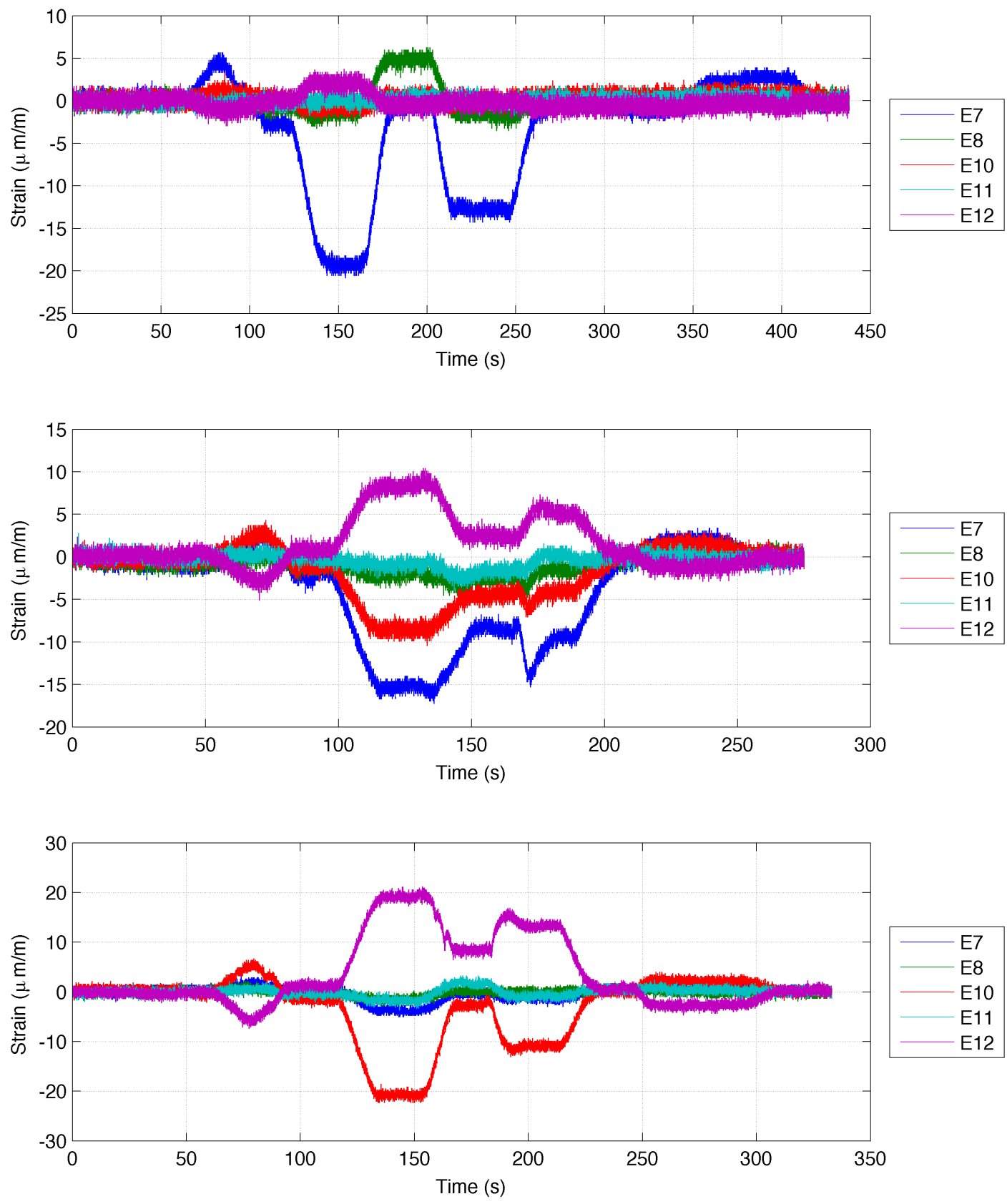

Figure A.2.: Recorded strains in the static load test in section S2. 

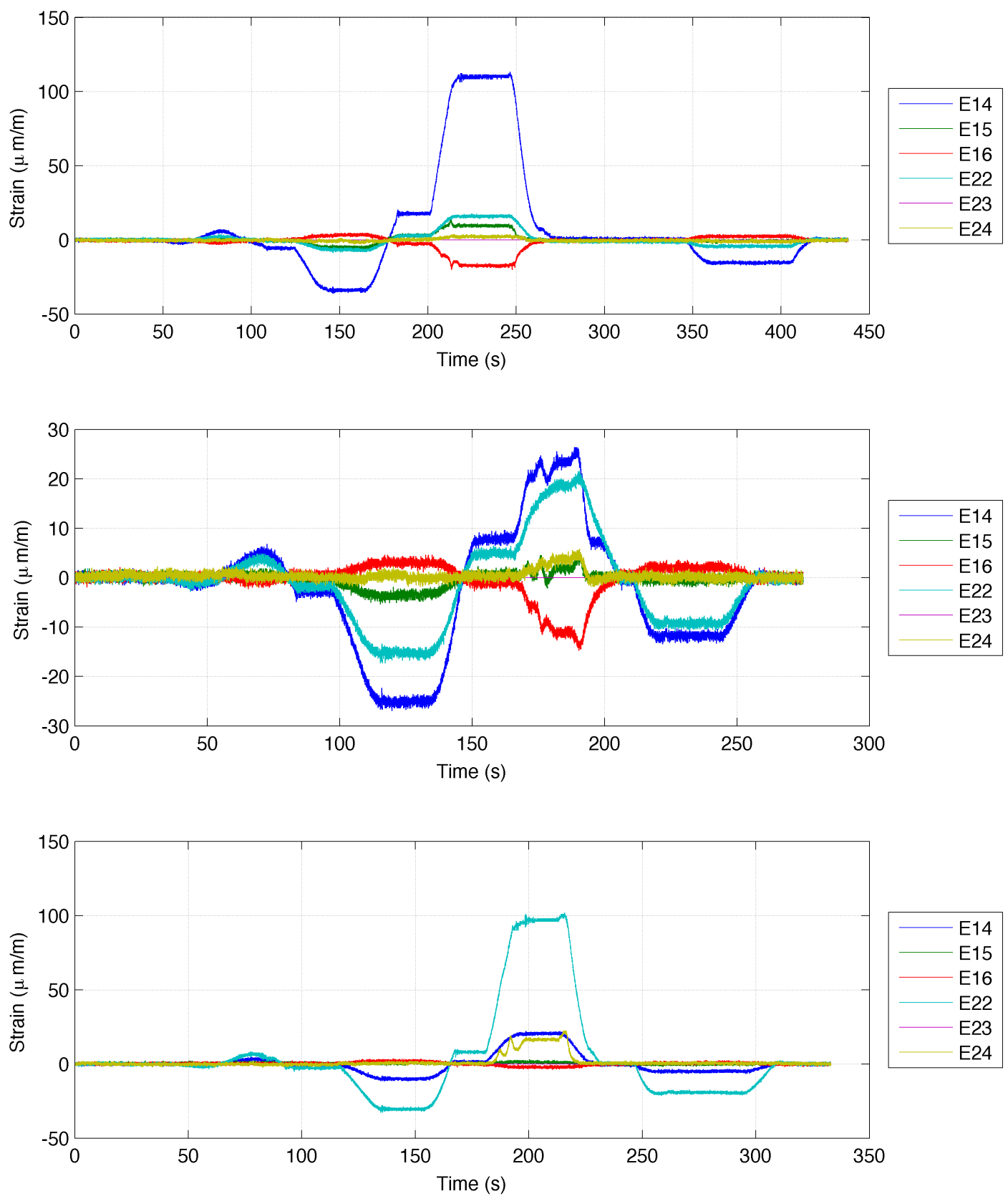

Figure A.3.: Recorded strains in the static load test in section S3. 

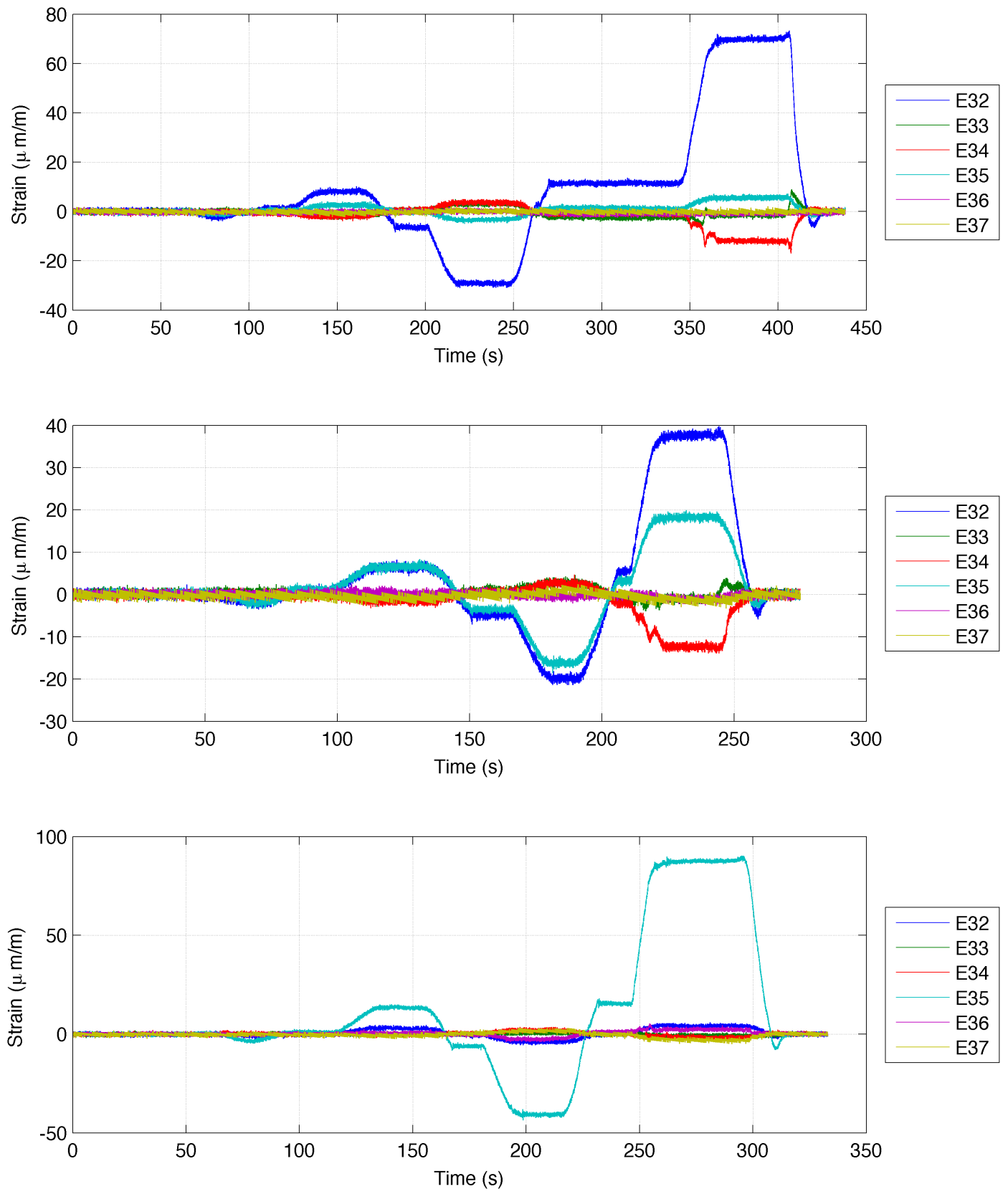

Figure A.4.: Recorded strains in the static load test in section S5. 

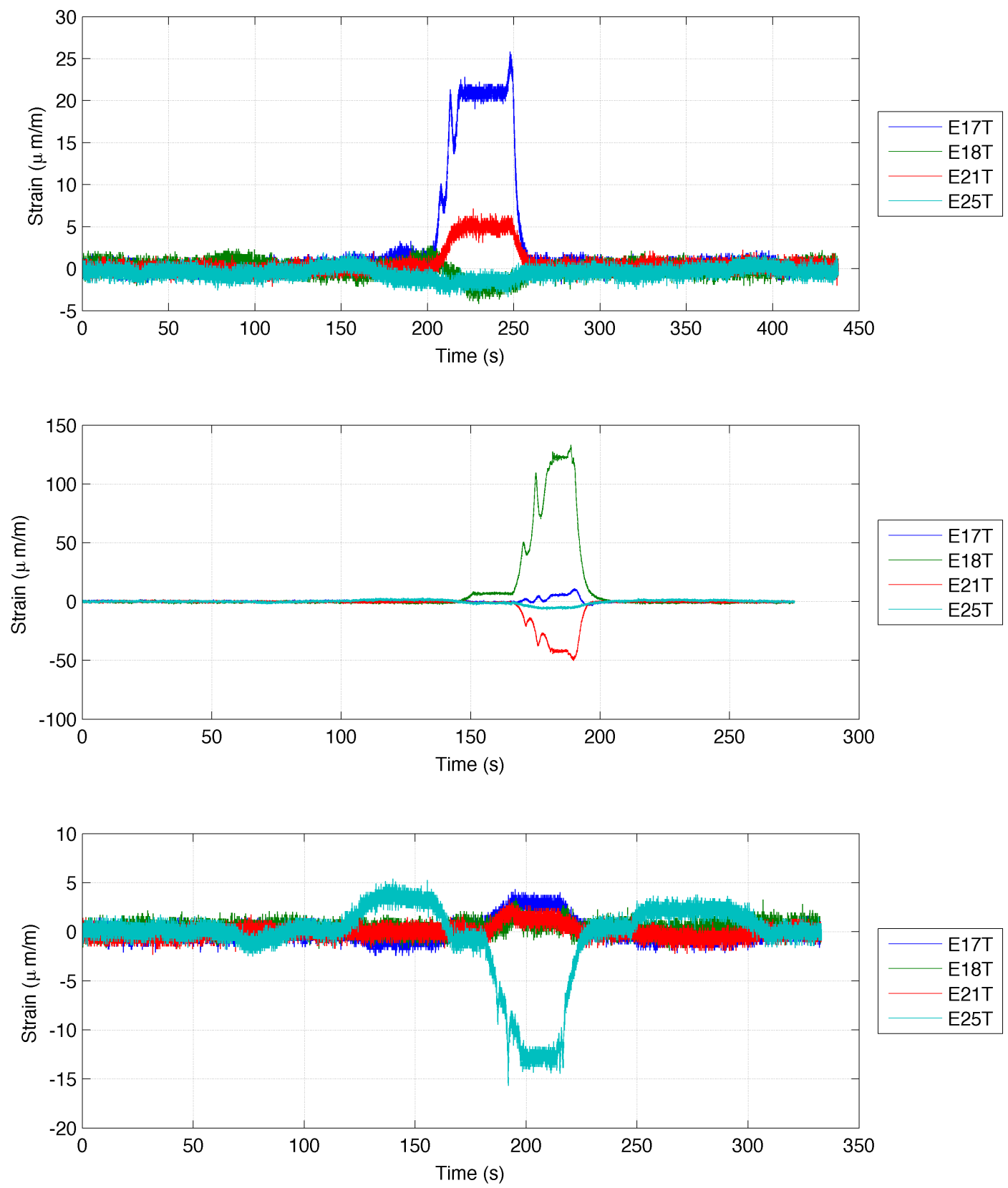

Figure A.5.: Recorded strains in the static load test at the deck (transverse direction at section S3). 


\section{A.3. Vibrating Wire Data}
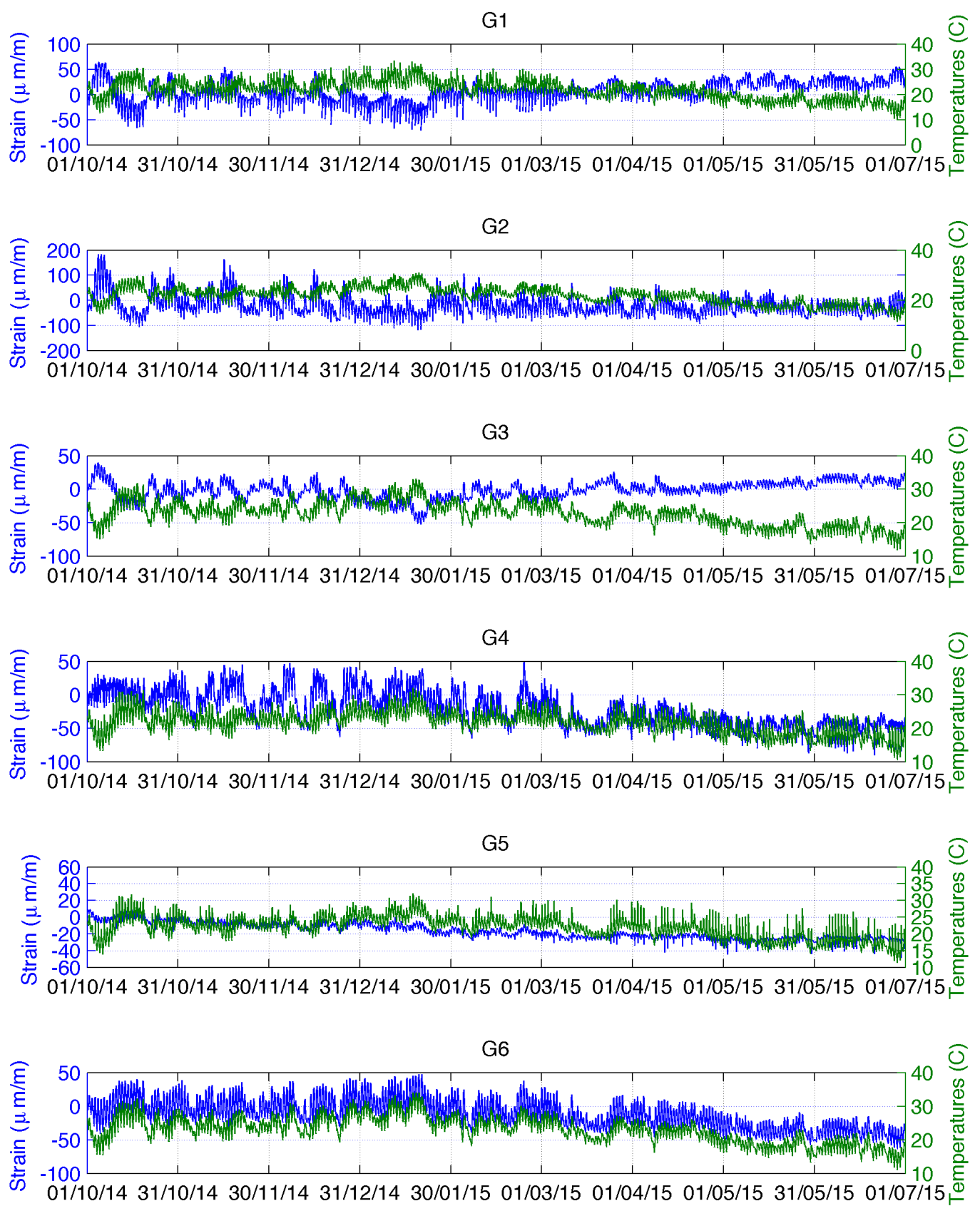

Figure A.6.: Temperatures and strains recorded at Section S1. 

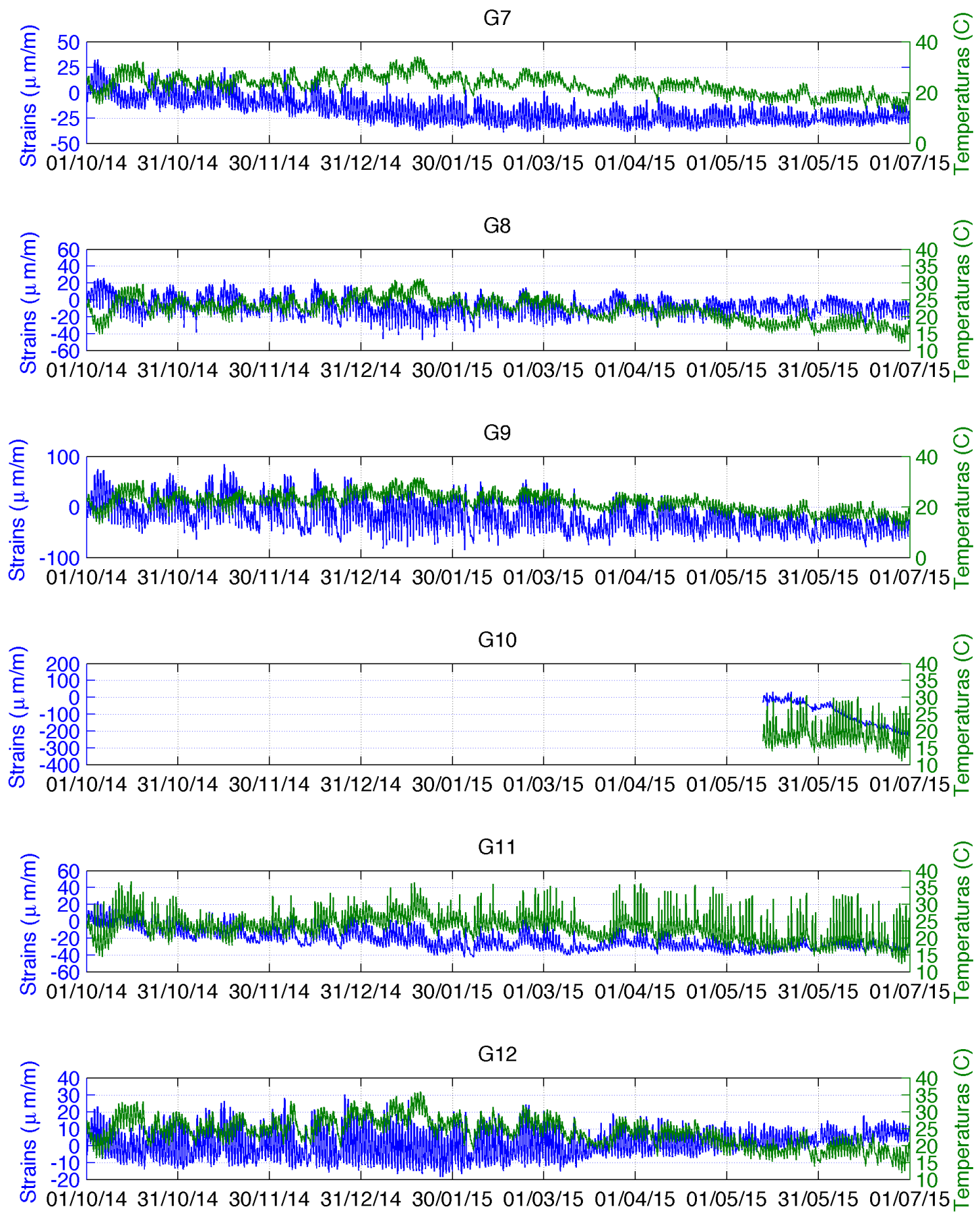

Figure A.7.: Temperatures and strains recorded at Section S3. 

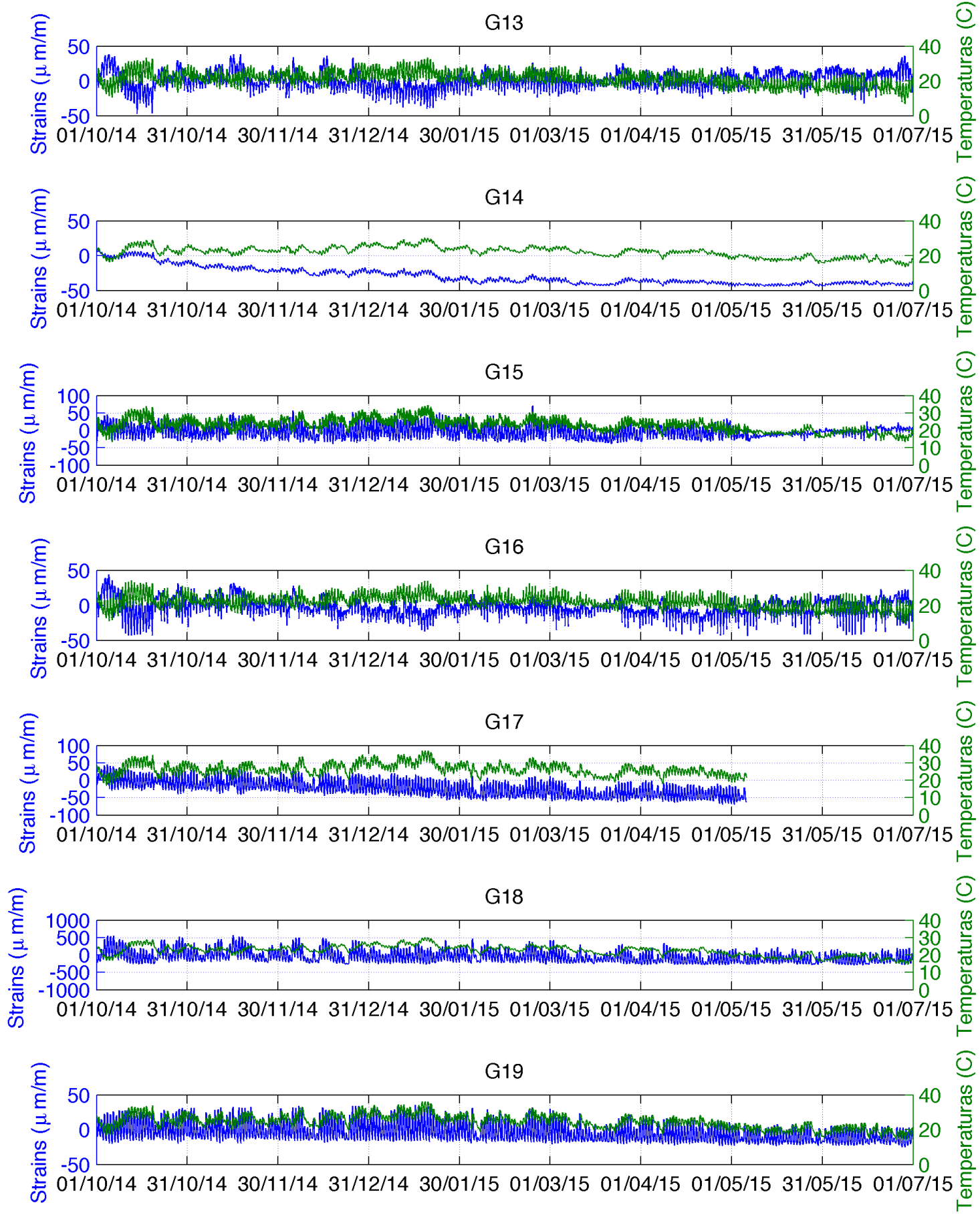

Figure A.8.: Temperatures and strains recorded at Section S5. 

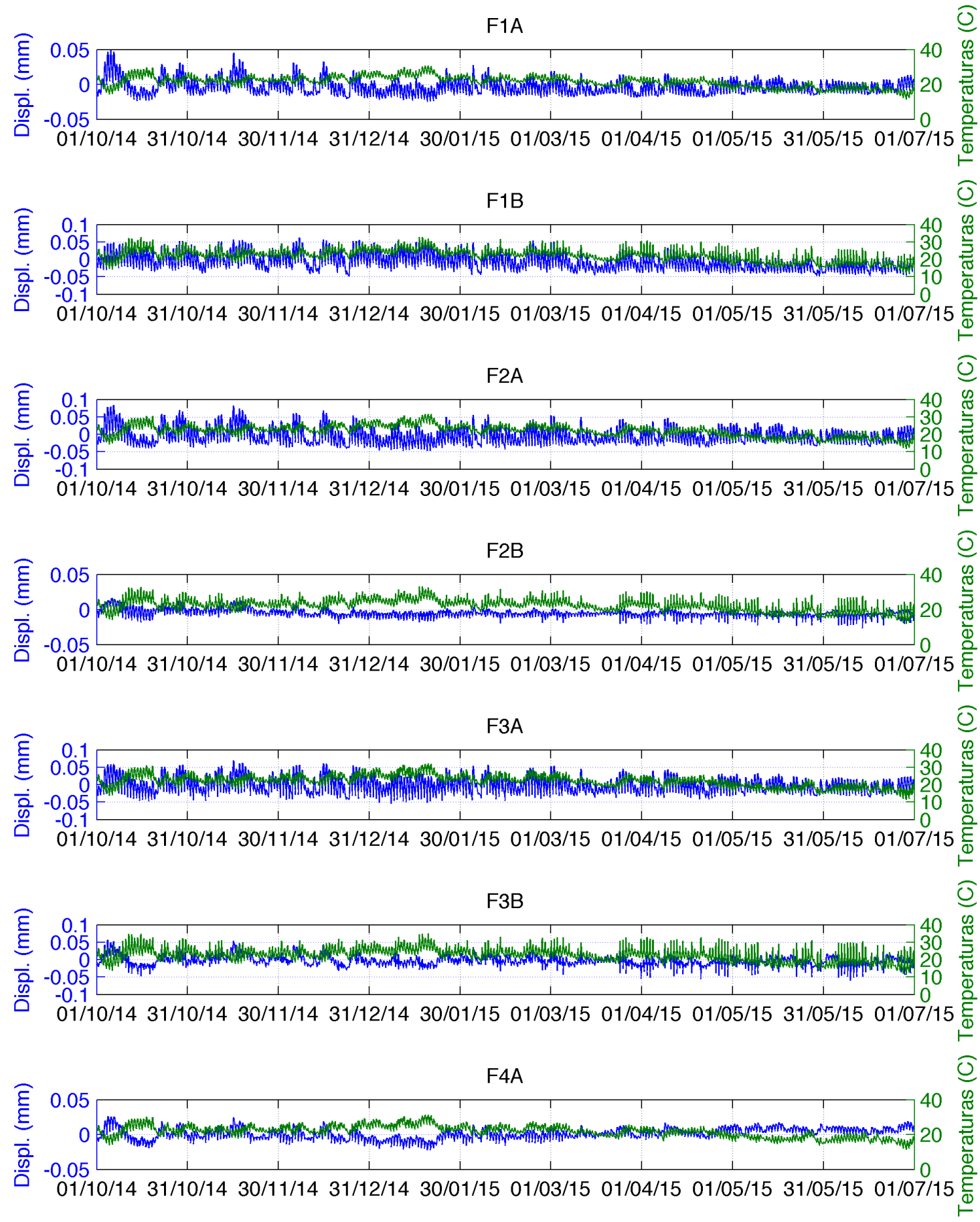

Figure A.9.: Temperatures and crack opening at the girders. 

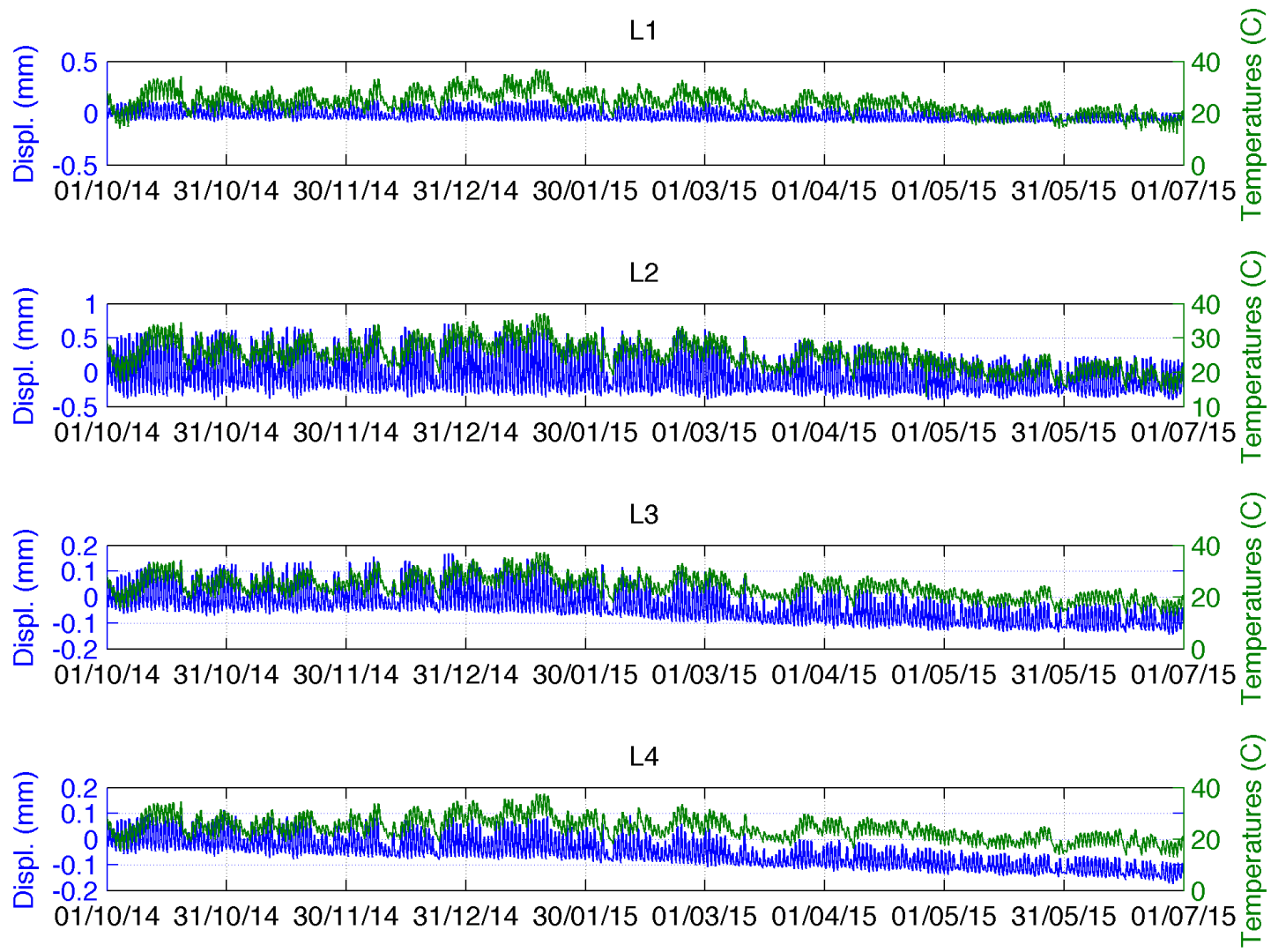

Figure A.10.: Temperatures and slab crack meter displacements. 


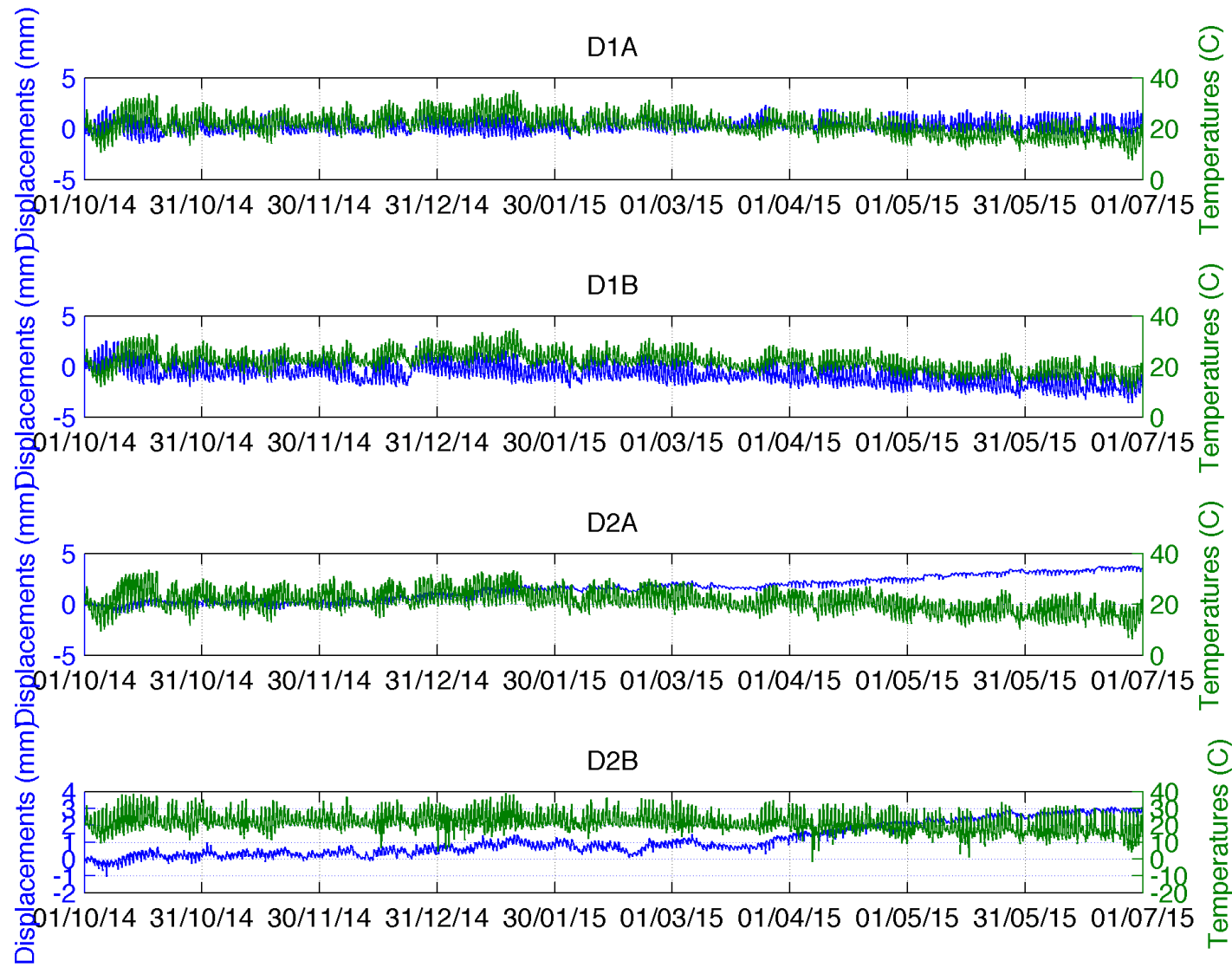

Figure A.11.: Temperatures and midspan displacements. 

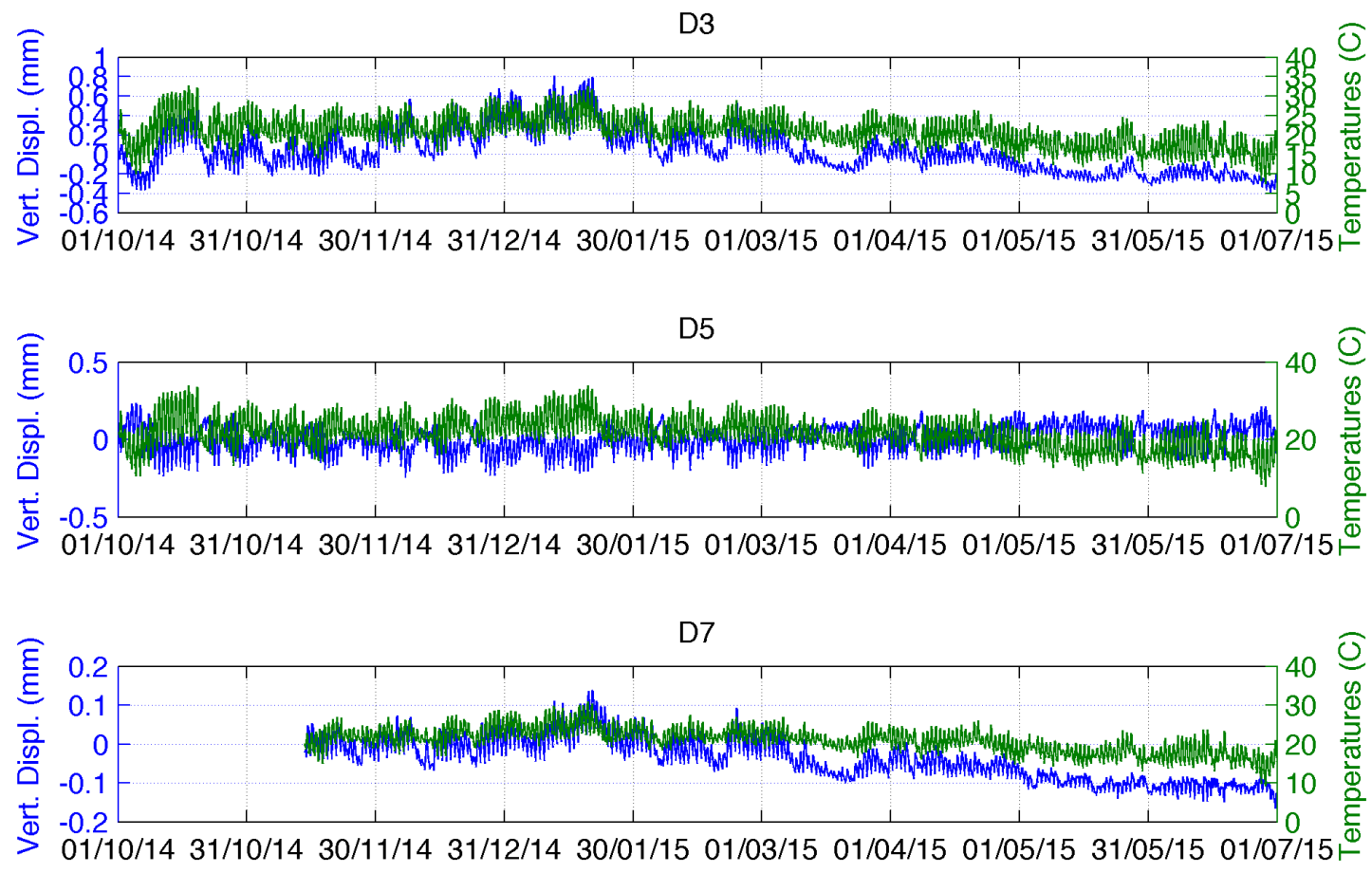

Figure A.12.: Temperatures and vertical displacements at supports.
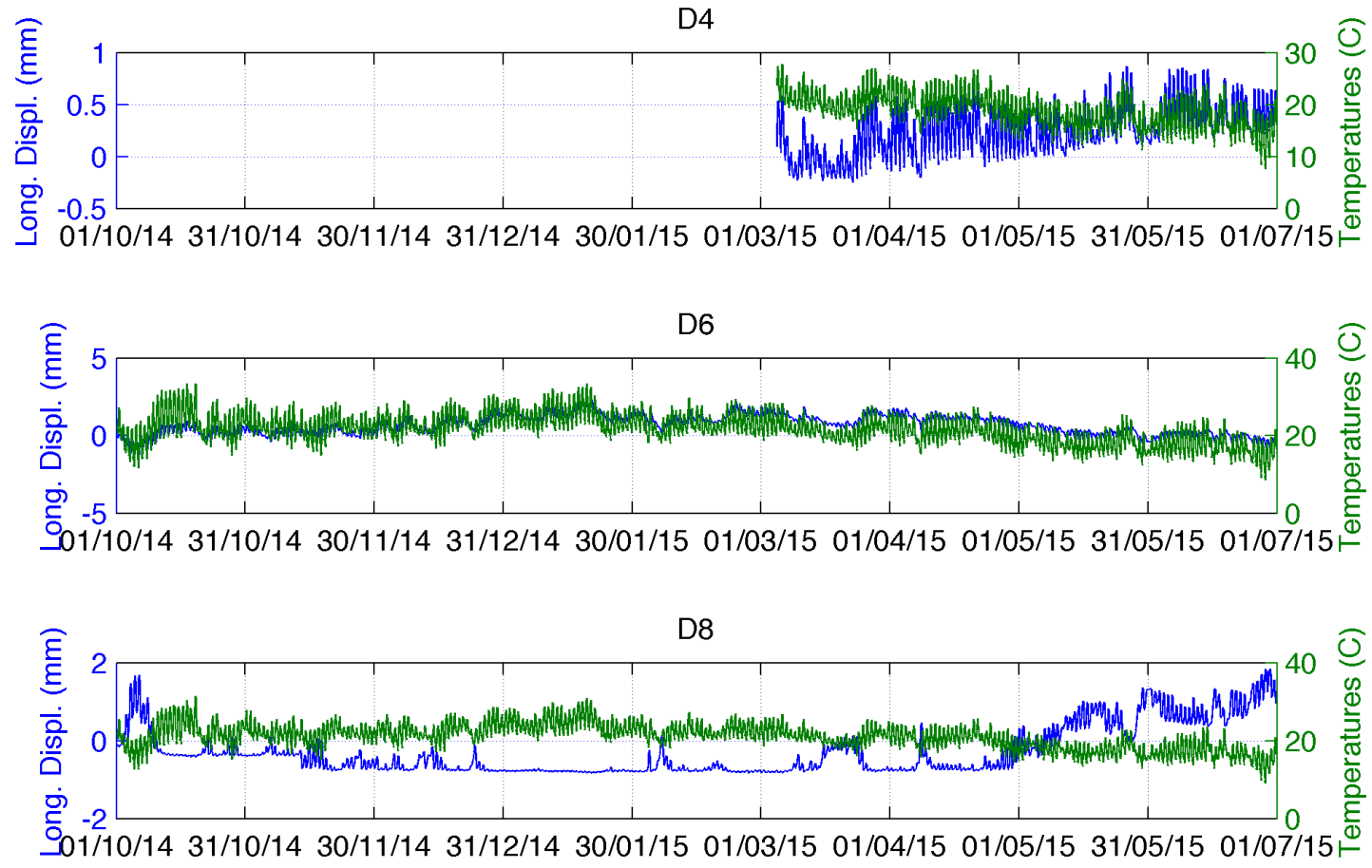

Figure A.13.: Temperatures and longitudinal displacements at supports. 

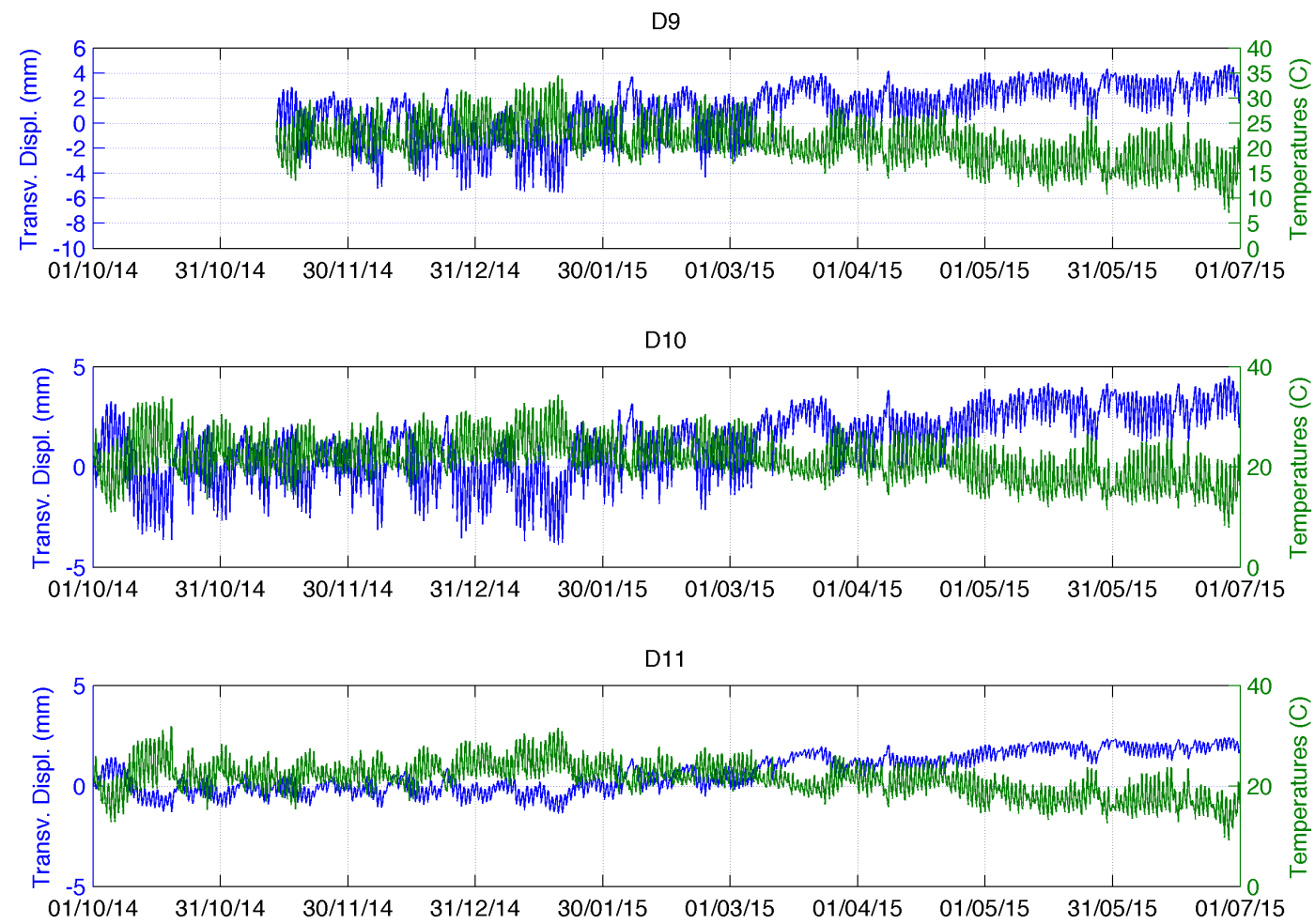

Figure A.14.: Temperatures and transverse displacements at supports. 